\title{
Bibliometrics: Help or Hoax for Quality?
}

\author{
F. Galetto \\ Department of Management and Production Engineering, Corso Duca degli Abruzzi, 24, 10129 TURIN , Italy \\ *Corresponding Author: fausto.galetto@fastwebnet.it
}

Copyright @ 2014 Horizon Research Publishing All rights reserved.

\begin{abstract}
Higher Education Institutions should provide students good teaching with good professors who have good knowledge of the matter they are teaching. Unfortunately, many times, the opposite happens: career leaps depend on "bibliometric indexes" (Impact Points, $h$-index, $s$-index, RG-index, $s$-index...).Until today, incompetent lecturers teach wrong ideas because they do not know Probability Theory and Reliability Theory. There is a must: professors must use the Scientific Approach when they teach, they must know what scientificness entails. Cases of incompetent teaching are presented: the Bass model, invented by Bass in 1969 , is used here to show how Bass itself made errors in the first time, and later, many professors copied, with irrational attitude, his ideas and diffused them to much more many students, all over the world. The same is for the fuzzy theory applied to Quality, for inventory management, for six sigma.
\end{abstract}

Keywords Success-Index, Bass Model, Fuzzy Logic, Linguistic Variables, Inventory Management, Six Sigma, Reliability, Quality, Scientific Approach

\section{Introduction}

Higher Education is seen many times as a Production System, and students are considered as its "Customers". Books and magazines are suggested to students, attending "Quality Courses" at Universities. Some of them are good some are not so good. Students use papers from magazines for their teaching; some papers have good Quality some are not very good. Therefore it seems important to stand-back a bit and meditate, starting from a managerial point of view.

In order to "measure" Quality (?) various bibliometric indices (e.g., $h$-index, $s$-index, Impact Points, RG-index, citations, ...) have been devised, based on informetric models. Research Quality (?) in many universities is based on these indexes: if you are cited many times you are a better professor than if you are not! That's the harsh reality...

Let's imagine that in one university there is a Quality Engineering Group (QEG, comprising four lecturers, all graduated CUM LAUDE, and teaching "Quality matters"; they are also in the ResearchGate with high Impact Points and $R G$-index!). Until today, incompetent lecturers teach wrong ideas because they do not know Probability Theory and Reliability Theory.Any rational person shall expect that those people will teach good ideas and will write "Quality papers on Quality matters". Do those people act correctly or wrongly? We will see it.

There is a must: professors must use the Scientific Approach when they teach, they must know what scientificness entails.

Therefore it seems important to stand-back a bit and meditate, starting from a managerial point of view. The author cannot solve this huge problem: the Universities Managment MUST solve it; the author can only use very fewcases of incompetent teaching: the Bass model, invented by Bass in 1969, to show how Bass itself made errors in the first time, and later, many professors copied, with irrational attitude, his ideas and diffused them to much more many students, all over the world. The same is for the fuzzy theory applied to Quality, for inventory management, for six sigma. The basic theories needed to understand are Probability, Reliability and Mathematics: nothing more is necessary.

\subsection{Some Preliminary Ideas}

They are very important, in the authors' opinion. If the redear wants to skip them he can do, at his own risk.

In order to show how those teachers failed to attain the university goal (i.e. to prepare students for the future,), the paper will use an application of the so called Six Sigma approach, in an automotive setting: a company [not Italian] making disc brakes needed important improvement and blinded by consultants decided to apply Six Sigma because (as they say) "6osolve every quality problem, allowing big savings for companies that use it".

One student to be graduated by a Turin university was appointed head of a team of five engineers and F. Galetto a "neutral observer" [actually he tried to let them work scientifically, BUT he did not succeed: they were deaf to any scientific influence, as we shall see]; the student attended the Quality Engineering Course [during his M. Sc. studies]: the lessons were provided by professors members of the "Quality Engineering Group (QEG)" (all graduated CUM LAUDE).

Is there any Quality in wrong teaching? Teaching must be scientific for future managers, as Deming and Galetto say 
(see the following figures).

We will use, as well, ideas of papers written by the QEG, and leave the reader to judge scientifically their contribution to Quality.

We provide here definitions found in books:

six sigma A quality program developed by Motorola Corporation with a detailed set of quality processes and tools.

Here, the definition of six sigma is built on the one offered in Linderman et al. (2003, p. 195). Writing in the prestigious Journal of Operations Management, those authors emphasized the need for a common definition of six sigma and proposed a definition paraphrased below:

Six sigma is an organized and systematic problem-solving method for strategic system improvement and new product and service development that relies on statistical methods and the scientific method to make dramatic reductions in customer defined defect rates and/or improvements in key output variables.

In the Six Sigma application they say that "6бsolve every quality problem, allowing big savings for companies that use it". Notice: processes, ... tools, ... problem-solving, ... new product development, ... statistical methods, ..., scientific method,... We shall see IF it is true. We first will analyse the scientificness, in the next section.

$6 \sigma$ became popular due to J. Welch (CEO of General Electric) who reported big saving in 1998!The DMAIC [Define-Measure-Analyse-Improve-Control] methodology is absolutely ineffective during product development of products and/or processes; it's sometimes useful for process improvement (see the real case).For product development of products and/or processes the DFSS [Design For Six Sigma] methodology is called; Taguchi fans search for "Robust Design". I met many of them: nobody knew anything about Reliability!!!! (a very important dimension of Quality).ASI [American Supplier Institute] for DFSS propose five steps: Identify (project), Define (requirements), Develop (concept), Optimize (design), Verify (and launch), using TM [Taguchi Methods]. They claim that the new products are "better, faster and cheaper, with more reliability, lower warranty costs, higher market share", measuring everything with the SN ratio! I met many of them: nobody knew anything about Reliability, and Preventive Maintenance!!!!

\footnotetext{
signal-to-noise (SN) ratio analysis Analysis performed to determine the factor levels required to reduce variability and achieve the ideal function of a characteristic.
}

See the stupid ideas of Montgomery! Compare this with FAUSTA VIA (see later)... and think!

Others points are related to s-index, Fuzzy Theory and Control Charts, Inventory Management, ....

In order to show how teaching fails to attain his goal (i.e. to prepare students for the future), the paper will use also a very spread model in Universities, the BASS Diffusion Model. At Turin Politecnico, at least seven lecturers teach this model, saying something like [we use "italic letters" for "reported sentences"]"The Bass model is a very useful tool for forecasting the adoption (first purchase) of an innovation (more generally, a new product)...A key feature of the model is that it embeds a <<contagion process $>>$ between those who have adopted the innovation and those who have not yet adopted it." The ideas [according to the Bass's Basement Research Institute] were given to P. Frevert, in Bass office, "The probability of adopting by those who have not yet adopted is a linear function of those who had previously adopted." He wrote down the mathematical expression of this idea in the form $\mathrm{f}(\mathrm{t}) /[1-\mathrm{F}(\mathrm{t})]=\mathrm{p}+\mathrm{qF}(\mathrm{t})$. He later, in 1969, published the paper [1] who has been one of the most cited papers, since then! In [1] that probability $\mathrm{f}(\mathrm{t}) /[1-\mathrm{F}(\mathrm{t})]=\mathrm{p}+\mathrm{qF}(\mathrm{t})$ is named "likelihood" (why?).

At Turin Politecnico, in [2], you find "The Bass model is a centrepiece in the literature on new products...." and "The Bass model is a classic tool for the analysis of new products introduction from the marketing field...." and in some courses "The Bass model can be interpreted as a survival model (in the state of non-adopters) and is defined by a hazard function $\lambda(t)$, which is the probability of adopting the product in $(t, t+d t)$, under the condition of not having adopted up to $t$; if $f(t)$ is the probability density function of the random variable "time to adoption" and $F(t)$ the distribution function, $\lambda(t)=p+q F(t), \quad p=$ probability of innovative adoption, $q=$ probability of imitative adoption. "In [2], as well, (with other errors) one finds "...basically there are two probabilities of adoption and the second one $q$...; $p+q$ is the probability of adoption of the last customer."

Using Reliability Theory, the author will show that the previous divulgers do not know Probability Theory and provide their students wrong ideas; however, if their students would use their intelligence they could understand they got wrong ideas.

Is there any Quality in wrong teaching? Teaching must be scientific for future managers. Deming says (figure 1):

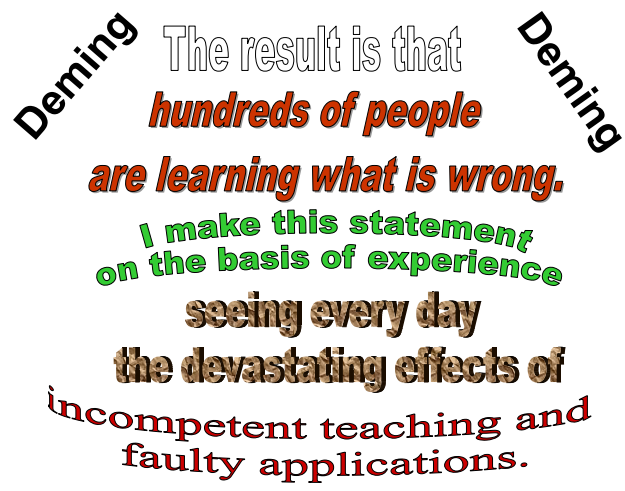

Figure 1. Deming ideas

Based on an idea by Kosmulski, who (2011) proposed to classify a paper as "successful" when receiving more citations than those made, the QEG (inAn informetric model for the success-index appeared on Scientometrics, 2012) proposed to classify a publication as "successful" when it receives more citations than a specific comparison term $(C T)$. We will see how wrong it is.

Let's now consider the method for Control Charts for Service, as given in the papers "On line Service Quality 
Control: the Qualitometro Method", (1998) Quality Engineering, 633-643, "Control Charts for linguistic variables: a method based on the use of linguistic quantifiers", (1998) International Journal Of Production Research, $n^{\circ} 16$ 99, 3791-3801, "Service Qualimetrics: the Qualitometro II Method", (99-2000) Quality Engineering, 13-20.

To understand F. Galetto findings it is important to provide immediately the ideas of the QEG [in the above papers]. Three excerpts of their papers are given.

$1^{\text {st }}$ Excerpt from the paper "On line Service Quality Control: the Qualitometro Method", Quality Engineering.

«Another delicate question is the numerical coding for judgements expressed on interview questionnaires. ... During data elaboration, scales are converted into numerical interval scales, and symbols are interpreted as numbers. ... The scalarization of collected data presents two main problems. The first is in introducing through coding an arbitrary metric, resulting in a wrong interpretation of gathered data; the second is a hidden assumption for an identical scale "interpretation" by any interviewed individual and a rigidity of this scale in time, especially for periodic service users. Scalarization may generate "distortion" effect, modifying the collected data partially or completely. ... In other words, the original information, "arbitrarily" enriched or directed to simpler aggregation and elaboration, may be highly modified if compared with the one really expressed by customers, with intuitable consequences.»

$2^{\text {nd }}$ Excerpt from "Control Charts for linguistic variables: a method based on the use of linguistic quantifiers", IJOPR.

«Ordered linguistic scales mainly differ from numeric or ratio scales because the concept of distance is not defined. The ordering is the main property attributed to such scales. For example, on a production line for fine liqueurs, a visual control of the corking and closing process might have the following possibilities: (a) 'reject', (b) 'poor quality', (c) 'medium quality', (d) 'good quality', (e) 'excellent quality'. The monitoring of production, using sampling control technique, is aimed at recognizing and, possibly, correcting unfavourable trends and out-of-control conditions. In order to do this the five classifications listed above could be attributed to some numerical values, leading to the construction, for example, of standard X-R charts. Although the numerical conversion of the verbal information simplifies subsequent analysis, it also gives rise to two problems. The first is concerned with the validity of encoding a discrete verbal scale into a numerical form. This approach introduces properties that were not present in the original linguistic scale (for example, is it legitimate to assume that the difference between the 'reject' state and the 'poor quality' state is the same as that between the 'medium' and 'high quality'?) [notice:'high quality' does not exist (in the "possibilities"!] Moreover, unlike scales used for physical measurements, ordered linguistic scales do not have either metrological reference standard or a measurement unit (QEG, 1995a, 1998). The second problem is related to the absence of consistent criteria for the selection of the type of numerical conversion. It is obvious that changing the type of numerical encoding may determine a change in the obtained results. Introducing arbitrary weight for quality categories may condition substantially the way of interpreting the process evolution. For example, if we assign to each quality level for a five level scale, the series of numbers: 1, 2, 3, 4, 5, or the series: -9, -3, 0, 3, 9, we obtain two different results. In this sense the analyst of the problem does directly influence the acceptance of results. Consequently, by attributing numbers to verbal information we might effectively [sic] move away from the original logic of the evaluator. In this way any conclusion drawn from the analysis on 'equivalent' numerical data could be partially or wholly distorted. The problems highlighted above can be alleviated if we resort to analysis tools able to avoid the arbitrary conversion of information. ... The fuzzy operator that is used in the paper allows for this flexibility in the decision logic.»

The "new" ideas of the QEG are in the paper "Ordered Samples Control Charts for Ordinal Variables" published in Quality and Reliability Engineering International [named by my students "the Qualitometro III Method"].The new three "tenors", of the QEG, say «The paper presents a new method for statistical process control when ordinal variables are involved. This is the case of a quality characteristic evaluated by on ordinal scale. The method allows a statistical analysis without exploiting an arbitrary numerical conversion of scale levels and without using the traditional sample synthesis operators (sample mean and variance). It consist of different approach based on the use of a new sample scale obtained by ordering the original variable sample space according to some specific 'dominance criteria' fixed on the basis of the monitored process characteristics. Samples are directly reported on the chart and no distributional shape is assumed for the population (universe) of evaluations".»

As usual (as in Qualitometro I and in Qualitometro II) the new "tenors" add: «Many quality characteristics are evaluated on linguistic or ordinal scales... ... The levels of this scale are terms such as 'good', 'bad', 'medium', etc..., which can be ordered according to the specific meaning of the quality characteristic at hand. Ordered linguistic scales mainly differ from numerical or cardinal scales because the concept of distance is not defined. The ordered is the main property associated to such scales. ... The problem of on-line monitoring of ordinal quality characteristic required the development of techniques able to deal with ordinal data. The assignment of weights, demerits and so on, to reflect the degree of severity of product non-conformity, has been adopted in many circumstances. Different numbers of the demerits are assigned to each class and the total number of demerits is monitored by some control chart for defectives. This is a subjective approach that requires the ability to uniquely classify each state into of several mutually exclusive classes, with well-defined boundaries among them. »... If one decides to use numbers two problems arise (as in Qualitometro I and as in Qualitometro II): «the first is 
concerned with the validity of encoding a discrete verbal scale into a numerical form. The numerical codification implies fixing the distances among scales levels, thus converting the ordinal scale into a cardinal one; the second is related to the absence of consistent criteria for the selection of the type of numerical conversion. It is obvious that changing the numerical encoding may determine a change in the obtained results. In this way the analyst directly influences the acceptance of results. Therefore, any conclusions drawn from the analysis on 'equivalent' numerical data could be partially or wholly distorted»)(as in Qualitometro I and in Qualitometro II).

Also books, used in universities, have the same problems.

We consider now the case of inventories, as given in Brandimarte, P., Zotteri G. (2006) Introduction to Distribution Logistics, STATISTICS IN PRACTICE, Wiley Interscience and Hopp W., J., Spearman M., L.,Factory Physics (2nd ed.), McGraw-Hill Higher Education.

This can be related to "preventive replacement before out-of-stock".

Inventories are stockpiles of raw material, supplies, components, work in progress and finished goods that appear at numerous points throughout a firm's production and logistic channel.

Having these inventories on hand cost at least $20 \%$ of their value per year.Therefore, carefully managing inventory levels makes good economic sense, because in recent years the holding of inventories has been roundly criticised as unnecessary end wasteful. Actually good management of inventories improve customer service and reduce costs.

To show F. Galetto ideas we consider here "stockout", or "type I Service" model, as I found it in books and papers (more details on the probabilistic behaviour are given later). The costs involved are:

$\mathrm{A} \lambda / \mathrm{Q}$, the "average" order cost per year

$\left(1-\mathrm{F}_{\mathrm{LT}}(R) \mathrm{p} \lambda / \mathrm{Q}\right.$, the cost of stockout [p is the cost per stockout, in euro] $\mathrm{F}_{\mathrm{LT}}(\mathrm{R})$ is the $\mathrm{CD}$ of demand during the lead time LT

$\mathrm{h}(\mathrm{R}+\mathrm{Q} / 2-\lambda \mathrm{LT})$, "average" inventory cost per year

being $\lambda$ the rate of demand.

Therefore "If stockouts are permitted and lead time is $L T>$ 0 , if demand is random, the formula to be minimised is the average cost rate $=$

$=\mathrm{A} \lambda / \mathrm{Q}+\mathrm{h}(\mathrm{R}+\mathrm{Q} / 2-\lambda \mathrm{LT})+\left(1-\mathrm{F}_{\mathrm{LT}}(R)\right) \mathrm{p} \lambda / \mathrm{Q}$ (where $R$ is the trigger quantity for launching the order $Q, p$ is the penalty for stockouts and $F_{L T}(d)$ is the probability distribution of the demand d, during the lead time $L T^{\prime \prime}$. Actually the formula is inconsistent, because it is based on an intuitive [not proved] extension of the formula for $<<$ "constant" demand, no stockouts permitted and lead time zero $>>$.Notice that this formula is the same as that in case of "everything known" and constant!

No scientific proof of the formula is ever provided!

Understanding that the formula is wrong is very easy.

$\left[1-\mathrm{F}_{\mathrm{LT}}(\mathrm{R})\right]$ is the probability that the demanded quantity $\mathrm{X}_{\mathrm{LT}}$, for the lead time $\mathrm{LT}$, is larger than $\mathrm{R}$ : $\mathrm{P}\left[\mathrm{X}_{\mathrm{LT}}>\mathrm{R}\right]$. That does not take into account that stockout occurs only if the replenishment order arrives LATE, later than all $\mathrm{R}$ units are sold! The probability of stockout depends on the competition of two stochastic processes: the demand versus the replenishment.Actually $\mathrm{A} \lambda / \mathrm{Q}+\mathrm{h}(\mathrm{R}+\mathrm{Q} / 2-\lambda \mathrm{LT})+\left(1-\mathrm{F}_{\mathrm{LT}}(R)\right) \mathrm{p} \lambda / \mathrm{Q}$ is an inconsistentformula.

We provide the basic ideas of Reliability Theory [13], [14], useful for the Bass model analysis and other methods (e.g. inventory, ...).

Let $\mathrm{T}$ be the random variable "Time to failure" of an item, and $0-\mathrm{t}$, the mission interval, whose duration is $\mathrm{t}$. The reliability $\mathrm{R}(\mathrm{t})$ is the probability that no failure happens in the mission, $\mathrm{f}(\mathrm{t})$ being the $\mathrm{pdf}$,

$$
R(t)=P[T>t]=1-F(t)=\int_{t}^{\infty} f(x) d x
$$

The mean of the r.v. is the Mean Time To Failure

$$
M T T F=E[T]=\int_{0}^{\infty} x f(x) d x=\int_{0}^{\infty} R(t) d t
$$

The failure rate, as any good student knows, is neither a (conditional) probability density nor a (conditional) probability; it is the ratio

$$
h(t)=f(t) / R(t)
$$

Hence it is easily derived that

$$
R(t)=\exp \left[-\int_{0}^{t} h(x) d x\right]
$$

When the failure rate is constant, failures are distributed "in the most random manner": the conditional reliability does not depend on past life.

It is easily seen that the knowledge of the failure rate $h(t)$ is enough to obtain any reliability characteristic $[R(t)$, MTTF, $\operatorname{MTTF}(t), F(t), f(t)]$.

The Mean Time To Failure, related to the interval $0-t$, is

$$
\operatorname{MTTF}(t)=\int_{0}^{t} R(x) d x
$$

In section 2, we provide some ideas on scientificness.

\section{2. "Scientificness"}

We provide the reader some ideas on the need of the scientific attitude that all the teachers must have [3].

$1^{\text {st }}$ Premise: Ever since he was a young student, at the secondary school, Fausto Galetto was fond of understanding the matters he was studying: understanding for learning was his credo $(\varphi \imath \lambda o \mu \alpha \theta \eta \varsigma \sigma u v i \eta \mu \mathrm{l})$; for all his life he was keeping this attitude, studying more than one ton of pages: as manager and as consultant he studied several methods invented by professors, but never he used the (many) wrong ones; on the contrary, he has been devising many original methods needed for solving the problems of the Companies he worked for, and presenting them at international conferences [where he met many bad divulgers, also professors "ASQC certified quality auditors"]; after 25 years of applications and experience, he became professor, with a dream "improve future managers (students) quality": the 
incompetents he met since then grew dramatically (also with documents. F. Galetto got from ERASMUS students (Fijiu Antony et al., 2001, Sarin S. 1997).

$2^{\text {nd }}$ Premise: "The wealth of nations depends increasingly on the quality of managers." (A. Jay [4]) and "Universities grow future managers." (F. Galetto)

Entailment: due to that, the author with will try, again, to send the important consequent message: let us, all of us, be scientific in all Universities, that is, let us all use our rationality. "What I want to teach is: to pass from a hidden non-sense to a non-sense clear." (L. Wittgenstein). End

"In my university studies ..., in most of the cases, it seemed that students were asked simply to regurgitate at the exams what they had swallowed during the courses." M. Gell-Mann "The Quark and the Jaguar..." [1994])\}. Some of those students later could have become researchers and then professors, writing "scientific" papers and books ... For these last, another statement of the Nobel Prize M. Gell-Mann is relevant: "Once that such a misunderstanding has taken place in the publication, it tends to become perpetual, because the various authors simply copy one each other. ".... >>, similar to "Imitatores, servum pecus" [Horatius, 18 B.C.] and "Gravior et validior est decem virorum bonorum sententia quam totius multitudinis imperitiae" [Cicero]. When they teach, "The result is that hundreds of people ... " [Deming (1986), fig. 1], because those professors are unable to practice maieutics [ $\mu \alpha \imath \varepsilon \cup \tau \imath \kappa \eta \tau \varepsilon \chi \nu \eta]$, the way used by Socrates for teaching [the same was for Galileo Galilei in his"Dialogue on the Two Chief World Systems"]. Paraphrasing P. B. Crosby, in his book "Quality is free", we could say"Professors may or may not realize what has to be done to achieve quality. Or worse, they may feel, mistakenly, that they do understand what has to be done. Those types can cause the most harm." What do have in common Crosby, Deming and Gell-Mann statements? The fact that professors and students betray an important characteristic of human beings: rationality [the "Adult state" of E. Berne (see fig. 2)]. Human beings are driven by curiosity that demands that we ask questions ("why? ..., why?") and we try to put things in order ("this is connected with that"): curiosity is one of the best ways to learn, but "learning does not mean understanding"; only twenty-six centuries ago, in Greece, people began to have the idea that the "world" could be "understood rationally", overcoming the religious myths: they were sceptic [ $\sigma \kappa \varepsilon \pi \tau \mathrm{o} \mu \alpha_{l}=$ to observe, to investigate] and critic [ $\kappa \rho \imath \omega=$ to judge]: then and there a new kind of knowledge arose, the "rational knowledge".

Till today, after centuries, we still do not use appropriately our brain! A peculiar, stupid and terrific non-sense! During his deep and long experience of Managing and Teaching (more than 40 years), F. Galetto always had the opportunity of verifying the truth of Crosby, Deming and Gell-Mann statements.

To understand each other we need to define the word "scientific".

A document (paper or book) is "scientific" if it "scientifically (i.e. with "scientific method") deals with matters concerning science (or science principles, or science rules)". Therefore to be "scientific" a paper must both concern "science matters" and be in accordance with the "scientific method".

The word "science" is derived from the Latin word "scire" (to know for certain) (derived from the Greek words $\mu \alpha \theta \eta \sigma \imath \varsigma, \varepsilon \pi \imath \sigma \tau \eta \mu \eta$, meaning learning and knowledge, which, at that time, were very superior to "opinion" [ $\delta \circ \xi \alpha]$, while today opinion of many is considered better than the knowledge of very few! \}; Knowledge is strongly related to

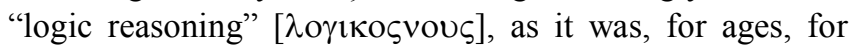
Euclid, whose Geometry was considered the best model of "scientificness". Common (good) sense is not science! Common sense does not look for "understanding", while science looks for "understanding"! "Understanding" is related to "intelligence" (from the Latin verb "intelligere" ([intus+legere: read into]: "intellige ut credas" i.e. understand to believe. Unfortunately "none so deaf as those that won't hear".

Let us give an example, the Pythagoras Theorem: "In a right triangle, the square of the length of the hypotenuse equals the sum of the squares of the lengths of the other two sides." Is this statement scientific? It could be scientific because it concerns the science of Geometry and it can be proven true by mathematical arguments. It is not-scientific because we did not specify that we were dealing with the "Euclidean Geometry" (based, among others, on the "parallel axiom": from this only, one can derive that the sum of the interior angles of a triangle is always $\pi$ ): we did not deal "scientifically" with the axioms; we assumed them implicitly.

So we see that "scientificness" is present only if the set of statements (concerning a given "system") are non-contradictory and deductible from stated principles (as the rules of Logic and the Axioms).

Let us give another example, the $2^{\text {nd }}$ law of Mechanics: "The force and the acceleration of a body are proportional vectors: $\mathrm{F}=\mathrm{ma}$, ( $\mathrm{m}$ is the mass of the body)". Is this statement scientific? It could be scientific because it concerns the science of Mechanics and it can be proven "true" by well-designed experiments. It is not-scientific because we did not specify that we were dealing with "frames of reference moving relatively one to another with constant velocity" [inertial frames (with the so called "Galilean Relativity": the laws of Physics look the same for inertial systems)] and that the speed involved was not comparable with the "speed of light in the vacuum [that is the same for all observers]" (as proved by the Michelson-Morley experiment: in the Special Relativity Theory, $\mathrm{F}=\mathrm{d}(\mathrm{mv}) / \mathrm{dt}$ is true, not $\mathrm{F}=\mathrm{ma}$ !) and not involving atomic or subatomic particles. We did not deal "scientifically" with the hypotheses; we assumed them implicitly. From the laws of Special Relativity we can derive logically the conservation laws of momentum and of energy, as could Newton for the "Galilean Relativity". For atomic or subatomic particles "quantum Mechanics" is needed (with Schrödinger equation as fundamental law).[12] 


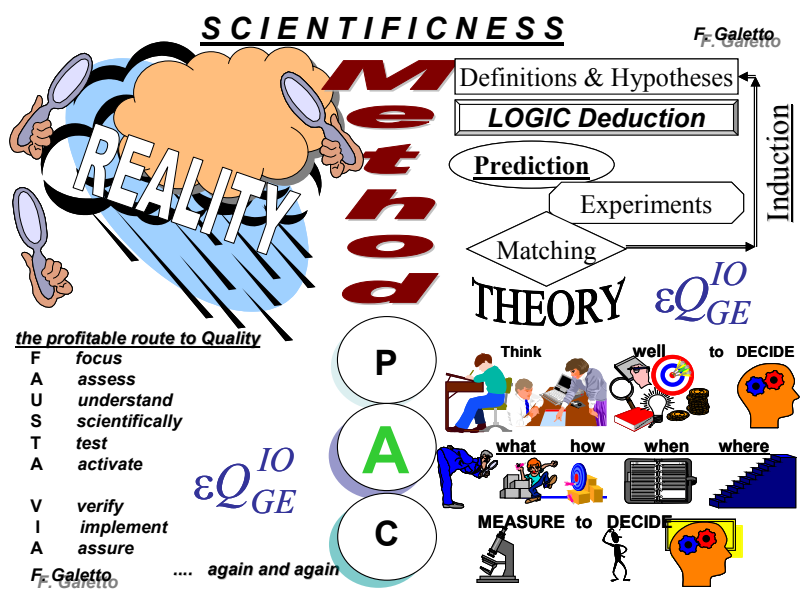

Figure 2. Scientificness

So we see that "scientificness" is present only if the set of statements (concerning a given "system") do not contradict the observed data, collected through well designed experiments ["scientific" experiments]: only in the XVII century, due to Galilei, Descartes, Newton, ... we learned that. Since that time only, science could really grow.

When we start trying to learn something, generally, we are in the "clouds"; reality (and truth) is hidden by the clouds of our ignorance, the clouds of the data, the clouds of our misconceptions, the clouds of our prejudices; to understand the phenomena we need to find out the reality from the clouds: we make hypotheses, then we deduct logically some consequences, predicting the results of experiments: if predictions and experimental data do match then we "confirm" our idea and if many other are able to check our findings we get a theory. To generate a theory we need Methods. Eric Berne [11], the psychologist father of "Transactional Analysis", stated that everybody interacts with other people through three states P, A, C [Parent, Adult, Child, (not connected with our age, fig. 2)]: the Adult state is the one that looks for reality, makes questions, considers the data, analyses objectively the data, draws conclusions and takes logic decisions, coherent with the data, methodically. Theory $[\theta \varepsilon \omega \rho 1 \alpha]$ comes from the Adult state! Methods $[\mu \varepsilon \theta 0 \delta$ o $\varsigma$ from $\mu \varepsilon \tau \alpha+o \delta$ o $\varsigma=$ the way through (which one finds out...)] used to generate a Theory come from the Adult state!

People who take for granted that the truth depends on "Ipse dixit" [ $\alpha \cup \tau o \varsigma \varepsilon \varphi \alpha$, "he said that" (F. Bass "said that", the QEG "said that", and published his ideas on a very important Management Magazine, "Management Science")], behave with the Parent state. People who get upset if one finds their errors and they do not consider them ["we are many and so we are right", they say!] behave with the Child state. [see the books of the Palo Alto group]

To find scientifically the truth (out of the clouds) you must Focus on the problem, Assess where you are (with previous data and knowledge), Understand Scientifically the message in the data and find consequences that confirm (or disprove) your predictions, Scientifically design Test for confirmation (or disproval) and then Activate to make the Tests. If you and others Verify you prediction, anybody can Implement actions and Assure that the results are scientific (FAUSTA VIA): all of us then have a theory. scientificness is there (fig. 2), (F. Galetto)

From these two examples it is important to realise that when two people want to verbally communicate, they must have some common concepts, they agree upon, in order to transfer information and ideas between each other; this is a prerequisite, if they want to understand each other: what is true for them, what is their "conventional" meaning of the words they use, which are the rules to deduce statements (Theses) from other statements (Hypotheses and "previous" Theses): rigour is needed for science, not opinions.

Many people must apply Metanoia $[\mu \varepsilon \tau \alpha v o r \alpha=$ change their mind (to understand)] to find the truth.

Here we accept the rules of Logic, the deductive Logic, where the premises of a valid argument contain the conclusion, and the truth of the conclusion follows from the truth of the premises with certainty: any well-formed sentence is either true or false. We define as Theorem "a statement that is proven true by reasoning, according to the rules of Logic"; we must therefore define the term True: "something" (statement, concept, idea, sentence, proposition) is true when there is correspondence between the "something" and the facts, situations or state of affairs that verify it; the truth is a relation of coherence between a thesis and the hypotheses. Logical validity is a relationship between the premises and the conclusion such that if the premises are true then the conclusion is true. The validity of an argument should be distinguished from the truth of the conclusion (based on the premises).

This kind of truth is found in mathematics.

Human beings evolved because they were able to develop their knowledge from inside (the deductive logic, with analytic statements) and from outside, the external world, (the inductive logic, with synthetic statements), in any case using their intelligence; the inductive logic is such that the premises are evidence for the conclusion, but the truth of the conclusion follows from the truth of the evidence only with a certain probability, provided the way of reasoning is correct.

The scientific knowledge is such that any valid knowledge claim must be verifiable in experience and built up both through the inductive logic (with its synthetic statements) and the deductive logic (with its analytic statements); in any case, a clear distinction must be maintained between analytic and synthetic statements.

This was the attitude of Galileo Galilei in his studies of falling bodies. At first time, he formulated the tentative hypothesis that "the speed attained by a falling body is directly proportional to the distance traversed"; then he deduced from his hypothesis the conclusion that objects falling equal distances require the same amount of elapsed time. After "Gedanken Experimente", Designed Experiments made clear that this was a false conclusion: hence, logically, the first hypothesis had to be false. Therefore, Galileo framed a new hypothesis: "the speed attained is directly proportional to the time elapsed". From 
this, he was able to deduce that the distance traversed by a falling object was proportional to the square of the time elapsed; through Designed Experiments, by rolling balls down an inclined plane, he was able to verify experimentally his thesis (it was the first formulation of the $2^{\text {nd }}$ law of Mechanics).

Such agreement of a conclusion with an actual observation does not itself prove the correctness of the hypothesis from which the conclusion is derived. It simply renders that premise much more plausible.

For rational people (like were the ancient Greeks) the criticism [ $\kappa \rho \imath v \omega=$ to judge] is hoped for, because it permits improvement: asking questions, debating and looking for answers improves our understanding: we do not know the truth, but we can look for it and be able to find it, with our

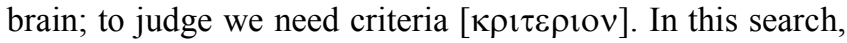
Mathematics [note $\mu \alpha \theta \eta \sigma r \varsigma$ ] and Logic can help us a lot: Mathematics and Logic are the languages that Rational Managers must know! Proposing the criterion of testability, or falsifiability, for scientific validity, Popper emphasized the hypothetic-deductive character of science. Scientific theories are hypotheses from which can be deduced statements testable by observation; if the appropriate experimental observations falsify these statements, the hypothesis is refused. If a hypothesis survives efforts to falsify it, it may be tentatively accepted. No scientific theory, however, can be conclusively established. A "theory" that is falsified, is no longer scientific.

"Good theories" are such that they complete previous "good" theories, in accordance with the collected new data.

A good example of that is Bell's Inequality. In physics, this inequality was used to show that a class of theories that were intended to "complete" quantum mechanics, namely local hidden variable theories, are in fact inconsistent with quantum mechanics; quantum mechanics typically predicts probabilities, not certainties, for the outcomes of measurements. Albert Einstein [one of the greatest scientists] stated that quantum mechanics was incomplete, and that there exist "hidden" variables that would make possible definite predictions. In 1964, J. S. Bell proved that all local hidden variable theories are inconsistent with quantum mechanics, first through a "Gedanken Experiment" and Logic, and later through Designed Experiments. Also, the great scientist, A. Einstein, was wrong in this case: his idea was falsified. We see then that the ultimate test of the validity of a scientific hypothesis is its consistency with the totality of other aspects of the scientific framework. This inner consistency constitutes the basis for the concept of causality in science, according to which every effect is assumed to be linked with a cause.

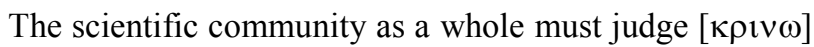
the work of its members by the objectivity and the rigour with which that work has been conducted; in this way the scientific method should prevail.

In any case, the scientific community must remember: Any statement (or method) that is falsified, is no longer scientific.
Here we assume that the subject of a paper is concerning a science (like Mathematics, Statistics, Probability, Quality

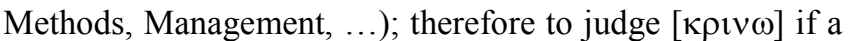
paper is scientific we have to look at the "scientific method": if the "scientific method" is present, i.e. the conclusions (statements) in the paper follow logically from the hypotheses, we shall consider the paper scientific; on the contrary, if there are conclusions (statements) in the paper that do not follow logically from the hypotheses, we shall not consider the paper scientific: a wrong conclusion (statement) is not scientific.

"To understand that an answer is wrong you don't need exceptional intelligence, but to understand that is wrong a question one needs a creative mind." (A. Jay). "Intellige ut credas".

Right questions, with right methods, have to be asked to "nature" (fig. 2)."Intellige ut credas".

It is easy to show that a paper, a book, a method, is not scientific: it is sufficient to find an example that proves the wrongness of the conclusion. When there are formulas in a paper, it is not necessary to find the right formula to prove that a formula is wrong: an example is enough; to prove that a formula is wrong, one needs only intelligence; on the contrary, to find the right formula, that substitutes the wrong one, you need both intelligence and ingenuity. I will use only intelligence and I will not give any proof of my ingenuity: this paper is for intelligence ... For example, it's well known (from Algebra, Newton identities) that the coefficients and the roots of any algebraic equation are related: it's easy to prove that $\pm \sqrt{-c / a}$ is not the solution (even if you do not know the right solution) of the parabolic equation $a x^{2}+b x+c=0$, because the system $\mathrm{x}_{1}+\mathrm{x}_{2}=-\mathrm{b} / \mathrm{a}, \mathrm{x}_{1} \mathrm{x}_{2}=\mathrm{c} / \mathrm{a}$ is not satisfied ( $\mathrm{x}_{1}$ and $\mathrm{x}_{2}$ are the roots).

The literature on "Quality" matters is rapidly expanding. Unfortunately, nobody, but me, as far as I know, [I thank any person that will send me names of people who take care ...], takes care of the "Quality of Quality Methods used for making Quality" (of product, processes and services). "Intellige ut credas".

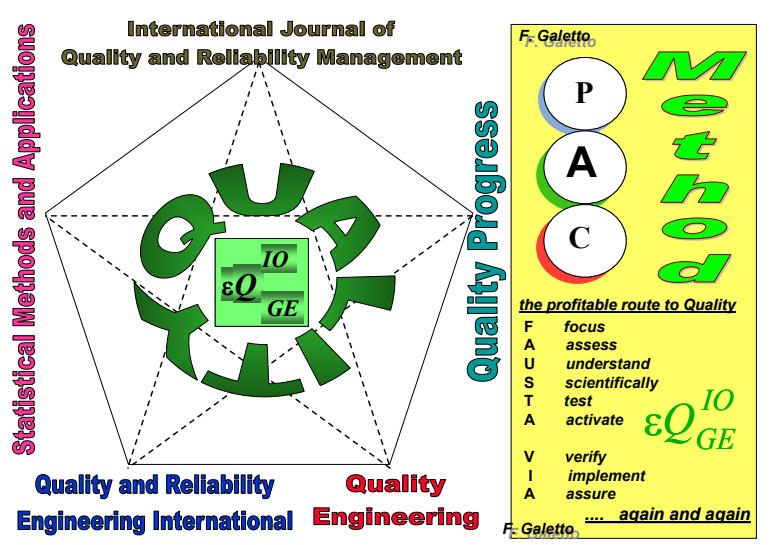

Figure 3. The "pentalogy"

F. Galetto is eager to meet one of them, fond of Quality as he is. If this kind of person existed, he would have agreed that "facts and figures are useless, if not dangerous, without 
a sound theory" (F. Galetto), "Management need to grow-up their knowledge because experience alone, without theory, teaches nothing what to do to make Quality" (Deming) because he had seen, like Deming, Gell-Mann and F. Galetto "The result is that hundreds of people are learning what is wrong. .... devastating effects of incompetent teaching and faulty applications." [Deming (1986)]

During 2006, F. Galetto experienced the incompetence of several people who were thinking that only the "Peer Review Process" is able to assure the scientificness of papers, and that only papers published in some magazines are scientific: one is a scientist and gets funds if he publishes on those magazines! Using the scientific method one can prove that the referee analysis does not assure quality of publications in the magazines of fig. 3 .

The symbol $\varepsilon Q_{G E}^{r o}$ [which stands for the "epsilon Quality"] was devised by F. Galetto to show that Quality depends, at any instant, in any place, at any rate of improvement, on the Intellectual hOnesty of people who always use experiments and think well on the experiments before actually making them (Gedanken Experimente) to find the truth" [Gedanken Experimente was a statement used by Einstein; but, if you look at Galileo life, you can see that also the Italian scientist was used to "mental experiments", the most important tool for Science; Epsilon $\left(^{\mathcal{E}}\right)$ is a Greek letter used in Mathematics and Engineering to indicate a very small quantity (actually going to zero); "epsilon Quality" conveys the idea that Quality is made of many and many prevention and improvement actions].

Many times F. Galetto spoiled his time and enthusiasm at conferences, in University and in Company courses, trying to provide good ideas on Quality and showing many cases of wrong applications of stupid methods [see references]. He will try to do it again ... by showing, step by step, one case (out of the hundreds he could document).... in order people understand that Quality is a serious matter. The Nobel price R. Feynman (1965) said that "for the progress of Science are necessary experimental capability, honesty in providing the results and the intelligence of interpreting them... We need to take into account of the experiments even though the results are different from our expectations." It is apparent that Deming, Feynman, and Gell-Mann are in agreement with $\varepsilon_{\varepsilon} Q_{G E}^{I O}$ ideas of F. Galetto. Once upon a time, A. Einstein said "Only two things are infinite: the Universe and the Stupidity of people; and I'mnot sure about the former". Let us hope that Einstein was wrong, this time. Anyway, before him, Galileo Galilei had said [in the Saggiatore] something similar "Infinite is the mob of fools".

All the methods, devised by F. Galetto, were invented and have been used for preventing and solving real problems in the Companies he was working for, as Quality Manager and as Quality Consultant: several million $€$ have been saved. Companies will not be able to survive the global market if they cannot provide integrally their customer the Quality they have paid for. So it is of paramount importance to know correctly what Quality means. Quality is a serious and difficult business; it has to become an integral part of management.

\section{Informetrics and the Success-Index}

Based on an idea by Kosmulski, who (2011) proposed to classify a paper as "successful" when receiving more citations than those made, the QEG (in An informetric model for the success-index appeared on Scientometrics, 2012) proposed to classify a publication as "successful" when it receives more citations than a specific comparison term $(C T)$. In the intention of the QEG authors $C T$ should be a suitable estimate of the number of citations that a publication - in a certain scientific context and period of time - should potentially achieve. According to this definition, the success-index is defined as the number of successful papers, among a group of publications examined, such as those associated to a scientist or a journal. QEG gave particular emphasis to a theoretical sensitivity analysis of the success-index (s-index).

For a generic group of papers, the $s$-index is defined as the number of papers with a number of citations greater than or equal to $C T_{i}$, i.e., a generic comparison term associated to the $i$-th publication: $C T_{i}$ is an estimate of the number of citations that a publication - in a certain scientific context and period of time - should potentially achieve. A score is associated to each of the $(P)$ publications of interest:

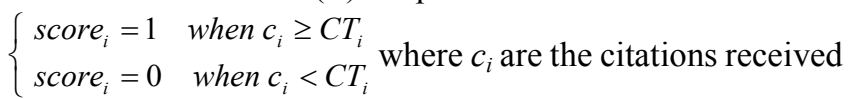
by the $i$-th publication. This achievement determines the condition of success.

The $s$-index is $=\sum_{i=1}^{P}$ score $_{i}$ QEG members consider a paper as a product coming out form a production process, and use one of the most popular informetrics, the law of Lotka $f(j)=K / j^{a}$ with $\mathrm{K}>0$ and $\mathrm{a}>1 . \mathrm{K}$ represents the number of articles with exactly 1 citation. In bibliometrics, according to its parameters, Lotka's law allows to describe the distribution of citations of different groups of papers. The QEG members (all graduated CUM LAUDE, teaching "Quality matters" and in the ResearchGate with high Impact Points!!!)model the $s$-index, assuming to analyse a homogeneous group of papers, i.e. a group of papers belonging to the same discipline of interest. Under this hypothesis the $C T$ is the same for all the papers; then we have $=\sum_{C T}^{\infty} K / j^{a}$.

The QEG than have a great idea [??]: they replace the integer variable $\mathrm{j}$ with a continuous variable $\mathrm{j}$ and then replace the sum with an integral $s=\int_{C T}^{\infty} k / j^{a} d j$ where $\mathrm{K}$ becomes $\mathrm{k}$ (due to the continuity). The QEG members (all graduated CUM LAUDE, and teaching "Quality matters") do that for finding some easy formulas by integration BUT they do not realise that they make a big mess. They find $s=\frac{k}{a-1} C T^{1-a} \quad$ and $P=\int_{1}^{\infty} k / j^{a} d j=\frac{k}{a-1}$ and then $s=P C T^{1-a}$ Since the QEG members do not know much Mathematics they do not recognise that $\mathrm{P}$ is proportional to the value of the "zeta" Riemann function at a: $\mathrm{P}=\mathrm{k} \zeta(a)$; therefore all the properties of the Riemann function apply, so 
proving that their QEG s-index is wrong. As a consequence all the calculations made by the integral formula are wrong. An alternative model, copying Egghe 2007, can be considering the total number $(\mathrm{C})$ of citations instead of the number $(P)$ of articles, which is the "mean" of the density; so $C=\int_{1}^{\infty} j k / j^{a} d j=\frac{k}{a-2}$ and $s=C(a-2) /(a-1) C T^{1-a}$.

After that, the QEG members make a sensitivity analysis of the model. Their natural question arising from the definition of the $s$-index is: "What does it happen varying $C T$ ?" They, treating $C T$ as a random variable with a certain standard deviation, try to see how the uncertainty related to its estimation propagates on the $s$-index. BUT they do more: they assume that the $s$-index can be seen as a non-linear function of three random independent variables: $C T, a$ and $P$ with expected values $\left(\mu_{C T}, \mu_{\alpha}, \mu_{P}\right)$ and standard deviations $\sigma_{C T}, \sigma_{\alpha}, \sigma_{P}$ of the three random variables.

Obviously the three random independent variables are NOT INDEPENDENT. Then the members of QEG revert to consider only the variable $\mathrm{CT}$; they assume an average value for "a" around 2 , and find $\sigma_{s}^{2} \cong\left(\mu_{P} / \mu_{C T}^{2}\right)^{2} \sigma_{C T}^{2}$. Then they assume that CT it is normally distributed (due to the central limit theorem, a great NONSENSE!) Moreover CT is not a continuous variable and the calculations of $\mathrm{s}$ are related to the "zeta" Riemann function $\zeta(a)$ and make simulations, with data $\mathrm{P}=718, \mathrm{C}=6641$ and $\mathrm{a}=2.12$, and with mean $\mu_{C T} \in\{10,11, \ldots, 30\}$ and standard deviation=1!!!

Citing the QEG (in the ResearchGate with high Impact Points!) and their findings, F. Galetto, according the QEG ideas, provided a "good" score to their paper, also if they make mathematical and statistical errors with calculation error as big as $10 \%$ !

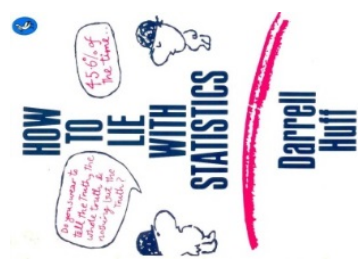

In the next section we shall see the implications of the Reliability concepts on the Bass model that it is taught to students, as shown in the introduction.

\section{The "Reliability" Bass Model}

We deal here with the Bass model, using the Reliability Theory.

The Bass model is used for studying the diffusion of products (but also of ideas, and of companies' certifications, ...).

Let $\mathrm{T}$ be the random variable "Time to purchase (diffusion of ...)" of a product, and $0-\mathrm{t}$, the time interval (whose duration is $t$ ), in which we want to make forecasting. $F(t)$ is the probability that a product is purchased before time $t$, while $R(t)$ is the probability that no purchase happens in the interval $0-\mathrm{t}$; $\mathrm{f}(\mathrm{t})$ is the pdf of the random variable "Time to purchase"; the formula that, according to Bass and his followers, provides "The probability of adopting by those who have not yet adopted is a linear function of those who had previously adopted." is, given, in the introduction, as

$$
\lambda(t)=f(t) /[1-F(t)]=p+q F(t)
$$

Comparing (6) and (3), it becomes evident that all those bad divulgers do not know Probability Theory: as a matter of fact, the $\lambda(\mathrm{t})$ dimensions are number/time [as for $\mathrm{f}(\mathrm{t})$, probability density function] and therefore $\lambda(\mathrm{t})$ is not a number, as a probability must be $[0 \leq \lambda(\mathrm{t}) \leq 1] ; \lambda(\mathrm{t})$ is neither a probability density function, as well, because, due to (4),

$$
\int_{0}^{\infty} h(x) d x=\infty
$$

Therefore all the people, all over the world, that claim that " $\lambda(t)$ is a probability ...", make a wrong statement. Are they "scientific" people?

\section{How Many Thousands are They, Bass Included?}

As a consequence, the parameters $\mathrm{p}$ and $\mathrm{q}$ in the Bass formulation of the model $\lambda(t)=p+q F(t)$ are not probabilities: they are not numbers; on the contrary they are numbers/time. $\lambda(\mathrm{t}), \mathrm{p}$ and $\mathrm{q}$ are "adoption rates", "purchasing rates", not probabilities!

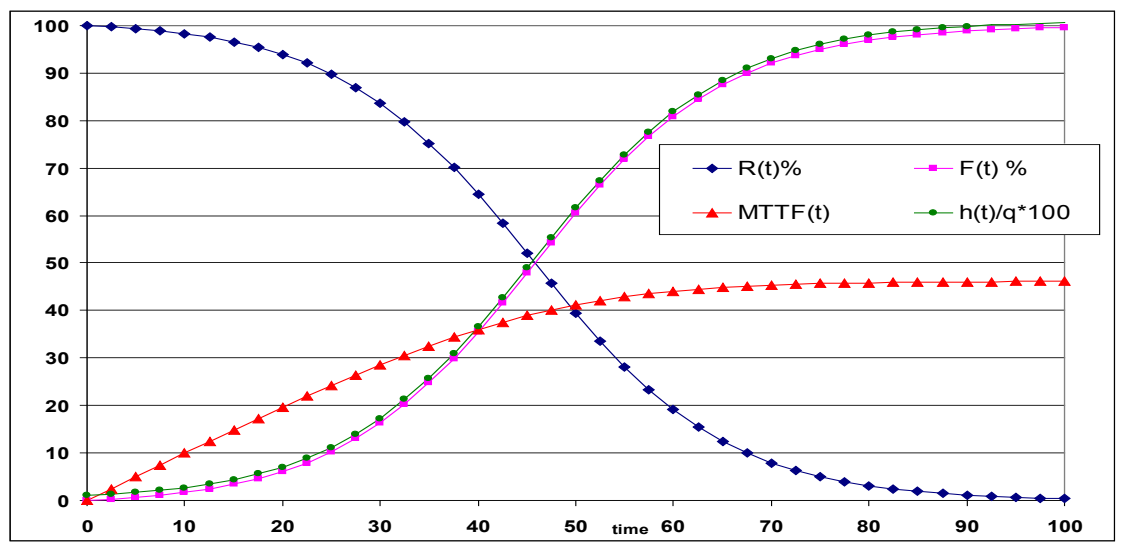

Figure 4. Shape of $\mathrm{F}(\mathrm{t}) \%, \ldots$, with $\mathrm{p}=0.001, \mathrm{q}=0.1$ 


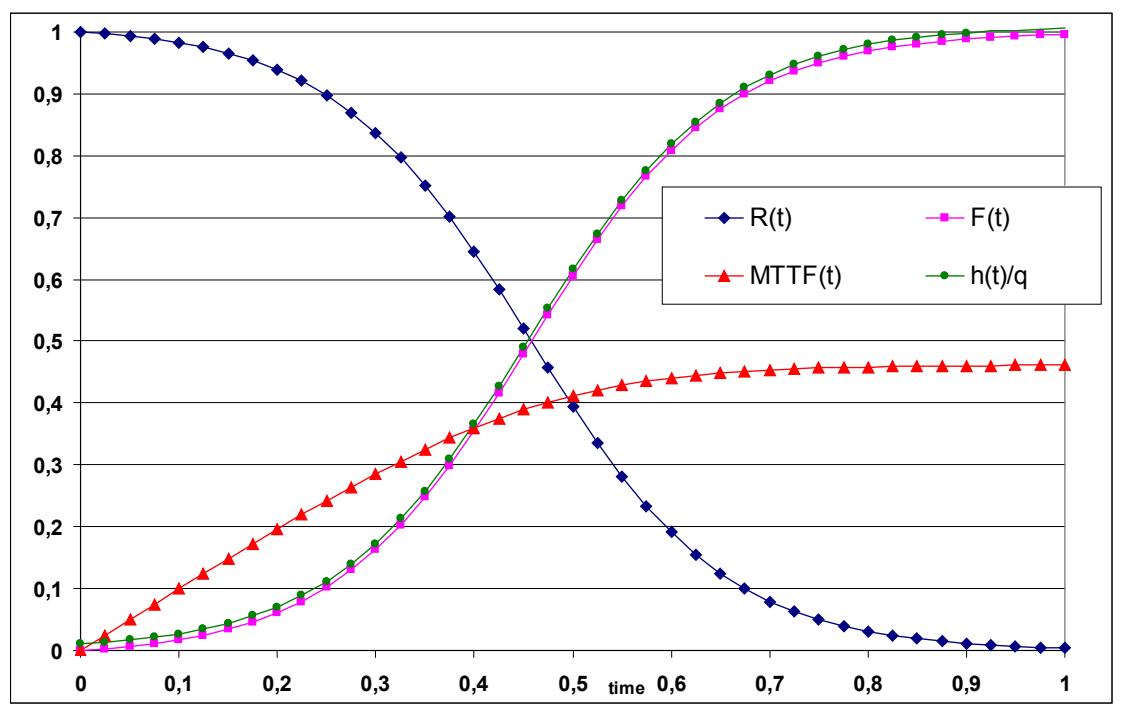

Figure 5. Shape of $\mathrm{F}(\mathrm{t}), \ldots$, with $\mathrm{p}=0.1, \mathrm{q}=10$

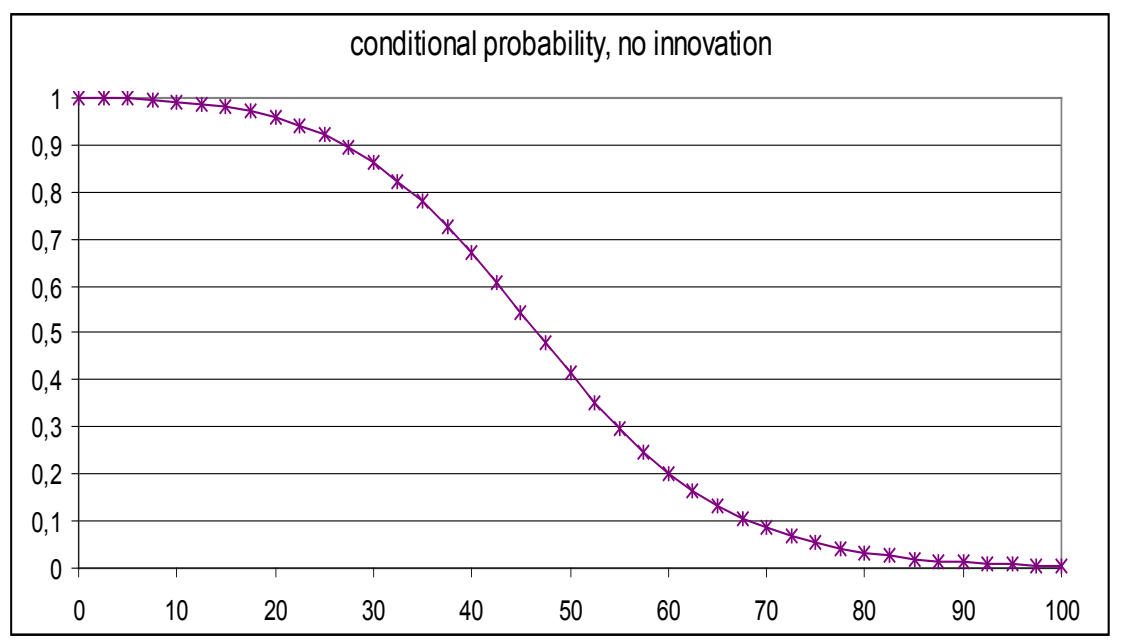

Figure 6. Conditional probability (no purchase)

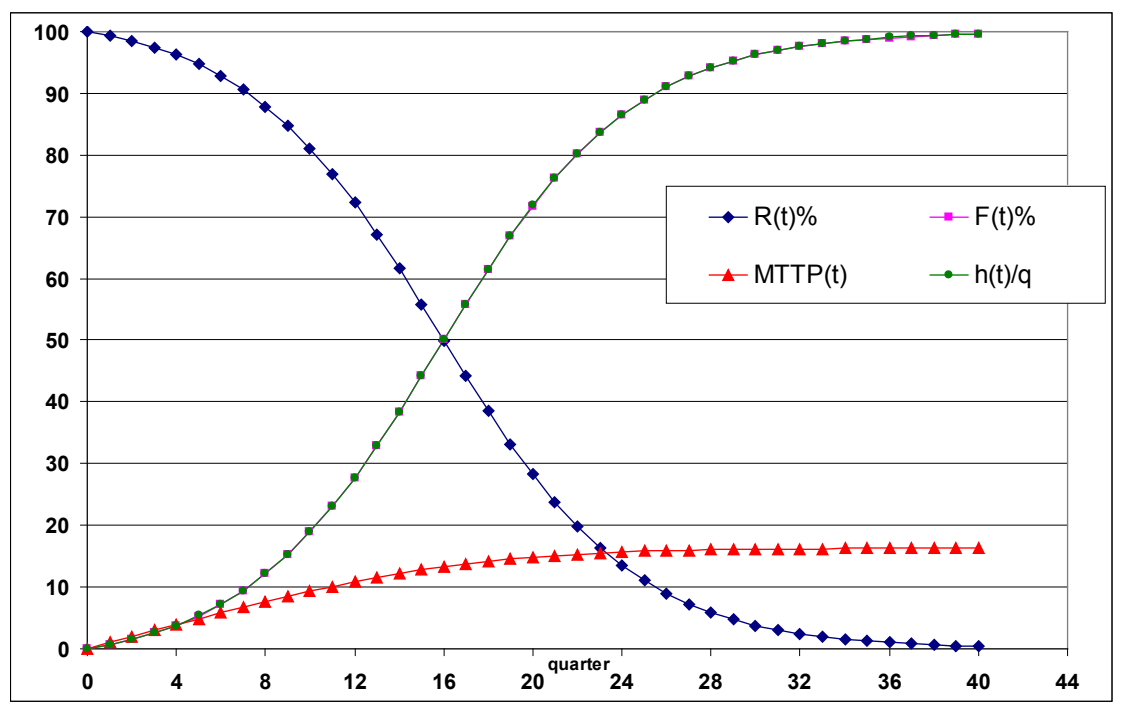

Figure 7. Shape of $\mathrm{F}(\mathrm{t}), \ldots$, with $\mathrm{p}=0.018$, $\mathrm{q}=0.67$ 
So the quantities $\lambda(t), p$ and $q$, can take any positive value, from 0 up to $\infty$, with dimension "time ${ }^{-1}$ ": e.g. 100000 adoptions/century! [which obviously is $<1$ if one use hours as time unit of measurement! but, nevertheless, $\lambda(t), p$ and $q$ are not probabilities].

In [2] one finds the "probabilities" $p=0.001$ and $q=0.1$, and there is no unit of measurement for time $\mathrm{t}$ \{we assume $\mathrm{t}$ measured by days [24 hours] $\}$. Figure 4 shows the graphs of the functions. Notice that $\mathrm{F}(\mathrm{t})$ and $\mathrm{h}(\mathrm{t}) / \mathrm{q}$ have a very similar shape, and $\operatorname{MTTP}(\mathrm{t})$ [indicated as $\operatorname{MTTF}(\mathrm{t})]$ tends to $\mathrm{E}[\mathrm{T}]$.

Using, on the contrary, the "rates" $\mathrm{p}=0.1 /[100$ days $]$ and $\mathrm{q}=10 /[100$ days], and time $\mathrm{t}$ measured by 2400 hours [0.1, in fig. 5], one gets the same kind of shape; actually any formula depends on the products [pure numbers!] $\mathrm{p}^{*} \mathrm{t} . \mathrm{q}^{*} \mathrm{t}$ and q*MTTP(t).

We name Mean Time To Purchase the mean of the random variable $\mathrm{T}$,

$$
M T T P=E[T]=\int_{0}^{\infty} x f(x) d x=\int_{0}^{\infty} R(t) d t
$$

The purchasing (diffusion, ...) rate is, obviously, neither a (conditional) probability density nor a (conditional) probability; it is [see (6)]

$$
h(t)=f(t) / R(t)
$$

The knowledge of the purchasing rate $h(t)$ is enough to obtain any purchasing characteristic $[\mathrm{R}(\mathrm{t})$, MTTP, MTTP $(\mathrm{t})$, $\mathrm{F}(\mathrm{t}), \mathrm{f}(\mathrm{t})]$.

The Mean Time To Purchase, related to the interval $0-\mathrm{t}$, is

$$
\operatorname{MTTP}(t)=\int_{0}^{t} R(x) d x
$$

Now using the hypothesis of professor F. Bass, one gets from (6) the non-linear differential equation

$$
f(t)=[p+q F(t)][1-F(t)]
$$

Using $R(t)$, the probability that no purchase happens in the interval $0-t$, one gets from (6) and (10) the non-linear integral equation

$$
R(t)=\exp [-(p+q) t] \exp \left[q \int_{0}^{t} R(x) d x\right]
$$

whose solution is

$$
\operatorname{MTTP}(t)=(1 / q) \ln \left\{\frac{(p+q)}{p+q \exp [-(p+q) t]}\right\}
$$

The Mean Time To Purchase (diffusion, ...) is

$$
M T T P=\ln [(p+q) / p] / q=\ln [h(\infty) / h(0)] / q
$$

We can get a deep insight of the model by rewriting (12) in the form

$$
R(t)=\exp [-p t] \exp \{-q[t-M T T P(t)]\}
$$

From (15) we see that innovation (with innovation rate $p$, constant) exploits its exponential influence for the time t, while imitation (with imitation rate $\mathrm{q}$, constant) exploits its influence mainly for the time t-MTTP(t); the shape of the conditional probability $\mathrm{R}(\mathrm{t}) / \exp (-\mathrm{pt})$, the probability that no purchase happens in the interval $0-\mathrm{t}$, given that no innovation purchase happened in the interval, depends essentially on t-MTTP $(t)$ and on the imitation rate $q$; a typical shape is in figure 6. After MTTP, $\mathrm{R}(\mathrm{t})$ decreases exponentially and MTTP(MTTP) $\cong$ MTTP.

When the rate $\mathrm{p}$ is constant, all innovation purchases are distributed "in the most random manner": the conditional probability of no innovation purchase does not depend on the past innovation purchases of the product.

The point of maximum of $f(t)$ is found through the equation $R^{\prime \prime}(t)=0$, that is the value of the abscissa of the inflection point of $R(t)$.

Let us now use some data from the Bass paper [1].In [1] one finds the "plausible [according to Bass] probabilities" $p=0.018$ and $q=0.67$, and there is no unit of measurement for time $\mathrm{t}$ \{ since the forecast is by year, we assume $\mathrm{t}$ measured by years [4 quarters] $\}$. Figure 7 shows the graphs of the functions.

Notice that $F(t)$ and $h(t) / q$ have a "very very" similar shape (they cannot be seen apart), and MTTP(t) tends to E[T].

The peak is after 4 years (16 quarters); this is also the MTTP (in this case).

One can generalise the diffusion model by

$$
R(t)=\exp \left[-\int_{0}^{t} \alpha(x) d x\right] \exp \left[\int_{0}^{t} \beta(x) R(x) d x\right]
$$

where $\alpha(\mathrm{x})$ and $\beta(\mathrm{x})$ are any positive functions [rates] of time $\mathrm{x}$.

If advertising can influence the innovation purchases, it is logic not to consider $\mathrm{p}=$ constant, but, on the contrary, $\alpha(\mathrm{t})$ increasing in the first part of the life cycle of the product.

It is important to notice that the integral equation (16) [found by F. Galetto] is able to deal with several others diffusion models, e.g. the Verhulst (logistic growth) model [15], or Malthus (exponential growth) model [16], or Volterra (competitive growth) model [17].

We mention here some points of the conclusions of $F$. Bass [1]; he wrote: "The growth model developed in this [1] paper for the timing of initial purchase of new products is based upon an assumption that the probability of purchase at any time is related linearly to the number of the previous buyers. There is a behavioural rationale for this assumption. The model implies exponential growth of initial purchase to a peak and then exponential decay. In this respect it differs from other new product growth models." This Bass statement is a nonsense: it is enough looking at formula (15) and at figure 7: everything depends on the time interval "of initial purchase"!

Looking at figure 7 , it is evident that $F(t)$ is not exponential and therefore $\mathrm{f}(\mathrm{t})$ [its derivative is not exponential] from time 0 to the time of inflection point. Again, looking at figure 7, it is evident that $\mathrm{F}(\mathrm{t})$ is not exponential and therefore $\mathrm{f}(\mathrm{t})$ [its derivative is not exponential] from the time of inflection point up to $\infty$. Only after the time $t$ such that $M T T P(t) \cong M T T P$, one can see the "exponential decay"!

Bass model and its related errors were and have been, up to now, provided to students by professors who do not know 
Probability and Reliability Theory [but they have high informetric scores!!!].

Therefore students' learning is as in figure 1 (Deming)

Equally important is figure 8 , based on M. Gell-Mann ideas:

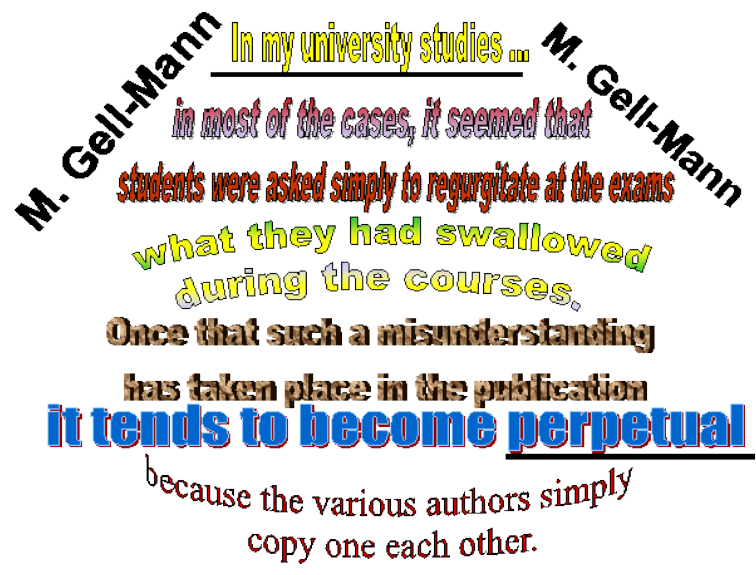

Figure 8. Gell-Mann ideas

We hope that professors will consider their duty to teach scientifically, in order to satisfy the learning need of their students and of the whole society.

\section{Qualitometro I/II/III: "Quality Engineering", and "Quality and Reliability Engineering International"}

In section 1 we provided citations to QEG members' papers, making their papers "good".

Before going on, let the reader ask himself: «if $F$. Galetto, acting as a scientist (as a $\mu \alpha \theta \eta \tau \eta \varsigma$ ), criticises the findings of "a member of the Editorial Board (as found in a book) of the journals Quality Engineering and International Journal of Quality and Reliability Management", what is the probability that the scientific community accept his ideas?»

Let's now consider the method for Control Charts for Service, as given in the QEG papers mentioned in section 1. To understand F. Galetto findings it is important to provide immediately the ideas of the QEG [in the above papers]. Three excerpts of their papers are given; the first two were given in section 1 .

$1^{\text {st }}$ Excerpt(see section 1)from the paper "On line Service Quality Control: the Qualitometro Method", Quality Engineering.

$2^{\text {nd }}$ Excerpt(see section 1)from "Control Charts for linguistic variables: a method based on the use of linguistic quantifiers", IJOPR.

QEG members were very unfortunate with their example regarding "a five level scale, the series of numbers: 1, 2, 3, 4, 5 , or the series: $-9,-3,0,3,9$, we obtain two different results.", because, due to the isomorphism, the two results are EQUIVALENT: since the data of fig. 10 can be used (for a 5 level scale) the result is 'medium quality' for each scale!!! "...1, 2, 3, 4, 5, or the series: -9, -3, 0, 3, 9, we obtain two different results." is a FALSE statement.

$3^{\text {rd }}$ Excerpt from the paper "Service Qualimetrics: the Qualitometro II Method", Quality Engineering.

"Questionnaires are used to perform service quality evaluation ... Proper scales for this purpose are linguistic scales. Linguistic scales used in the questionnaire possess the ordinal property only. The method ... is able to handle information expressed on linguistic scales without any artificial numeric scalarization. ... Refusal of an arbitrary encoding of scale levels means that a method must be devised for introducing an average operator on a scale where the distance concept ("norm") is not defined, only the ordering property is. ... The main novelty of the method are as follows: - No arbitrary and artificial encoding of collected information, •Aggregation of collected information using methods and tools proper for linguistic scale treatment.»

Reading those 3 papers $F$. Galetto understood [as a $\mu \alpha \theta \eta \tau \eta \varsigma]$ the following $1 *$ ) using numbers is wrong, and $2 \boldsymbol{A})$ it is correct ONLY to use fuzzy logic [Yager] and linguistic variables [Zadeh] in order to avoid the "tyranny of numbers", an important thing because "... forcing the decision maker to supply information with greater precision than he is capable of providing... may lead to incorrect answers ...".

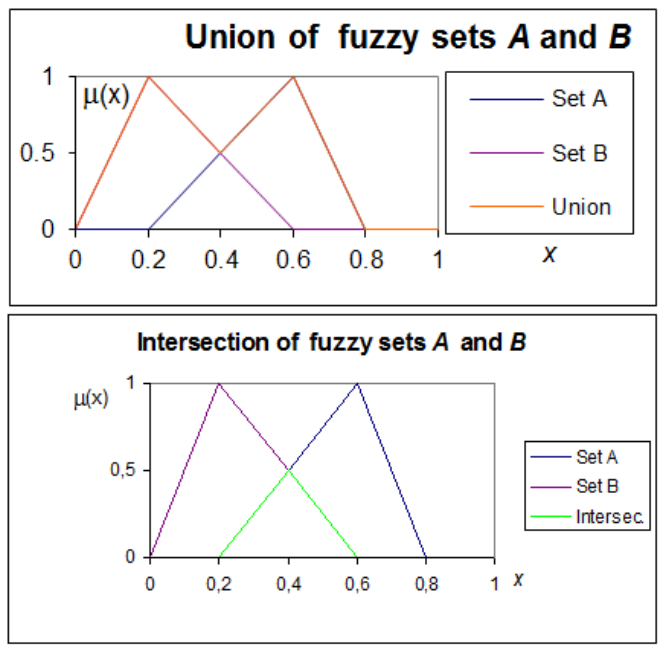

Figure 9. Operations on Fuzzy sets

What must do a serious scientist (a $\mu \alpha \theta \eta \tau \eta \varsigma$ ) when a new "theory" ["theory" (??) as Qualitometro pretends to be] is proposed? A serious scientist starts studying and understanding scientifically (fig .2 and FAUSTA VIA). That did Galetto: he studied fuzzy logic/linguistic very well.

Before going on the reader needs some basics of "Fuzzy Theory". We cannot provide them here due to allowed space limitations for the paper. (in fig. 9 we give only $\cap$ and $\cup$ operations. The GOM of the two fuzzy... are triangular)

It is important to remark here that all the "fuzzy operations" on the "fuzzy sets"are based on "classical operations on the classical mathematical functions as the GoM (Grades of Membership) are". [i.e. on the classical sets 
operations $\cap$ and $\cup$ !!!!]

We have used membership functions with triangular shape: this choice has been made only for the sake of simplicity; actually, the fuzzy literature shows many other classes of the membership functions.

It is logic that, from the above, one deduces that

"NO Grade Of Membership $\Rightarrow$ NO Fuzzy Theory".

In order to analyse correctly the "Qualitometro method" we need also the following information (see fig. 10).

As done by the QEG, in the Qualitometro method (see fig. 10), let's assume that "quality data" are collected on 8 "quality characteristics" $\mathrm{g}_{\mathrm{j}}$ [called "criteria"]; an evaluator provides an evaluation " $g_{j}(e)$ " to $j^{\text {th }}$ criterion ( $j^{\text {th }}$ "quality characteristic") and assigns an importance $I\left(g_{j}\right)$ to each evaluation criterion; both evaluation and importance are given on a 7 points linguistic scale [None, Very Low, Low, Medium, High, Very High, Perfect]. (see the Qualitometro Format, where some real data are presented; for example, the $1^{\text {st }}$ "quality characteristic" was rated as Very Low $=\mathrm{g}_{1}(\mathrm{e})$, with Importance graded as Low $\left.=\mathrm{I}\left(\mathrm{g}_{1}\right)\right)$. Many times the points linguistic scale $[\mathrm{N}, \mathrm{VL}, \mathrm{L}, \mathrm{M}, \mathrm{H}, \mathrm{VH}, \mathrm{P}]$ are transformed into numbers and therefore the "original linguistic" evaluations and importances provided by the evaluator, through a scalarization, are converted into numbers (e.g. 1, 2, 3, 4, 5, 6 ,7); then the numbers are used for any elaboration (e.g. expected values and control limits computation). This attitude is strongly criticised by QEG members who (for their "Qualitometro Method") say "It is worthwhile to emphasize that the provided aggregation does not perform any arbitrary scalarization of information given by evaluators on linguistic scales. ... Refusal of an arbitrary encoding of scale levels means that a method must be devised for introducing an average operator on a scale where the distance concept ("norm") is not defined ...".

They then aggregate the 8 criteria into a "quality" q, using (they say!) fuzzy logic with the formula $\mathrm{q}=\operatorname{Min}\left[\operatorname{Max}\left[\operatorname{NegI}\left(\mathrm{g}_{\mathrm{j}}\right), \mathrm{g}_{\mathrm{j}}(\mathrm{e})\right]\right]$, claiming that this formula is derived by "Fuzzy Theory" (as given in Yager).

Yager invented his method, capable of dealing with non-numeric data, in 1981, with the paper "A new methodology for ordinal multiobjective decision based on fuzzy sets". He stated that the decisions made by a Decision Maker (DM) are based on a certain number of "criteria" $\mathrm{g}_{\mathrm{j}}$; for each criterion the data are collected on a 7 points linguistic scale [None, Very Low, Low, Medium, High, Very High, Perfect]. Criteria can have different importance $\mathrm{I}\left(\mathrm{g}_{\mathrm{j}}\right)$. An evaluator [sometimes the Decision Maker (DM) himself] provides an evaluation " $\mathrm{g}_{\mathrm{j}}(\mathrm{e})$ " to $\mathrm{j}^{\text {th }}$ criterion and assigns an importance $I\left(g_{j}\right)$ to each evaluation criterion; both evaluation and importance are given on the same 7 points linguistic scale. The criteria are aggregated into a "decision function" $\mathrm{q}$ (called "quality" by the service quality evaluators), using fuzzy logic with formula $\mathrm{q}=\operatorname{Min}\left[\operatorname{Max}\left[\operatorname{NegI}\left(\mathrm{g}_{\mathrm{j}}\right), \mathrm{g}_{\mathrm{j}}(\mathrm{e})\right]\right]$ : QEG actually copied Yager(see Gell-Mann, fig. 8).

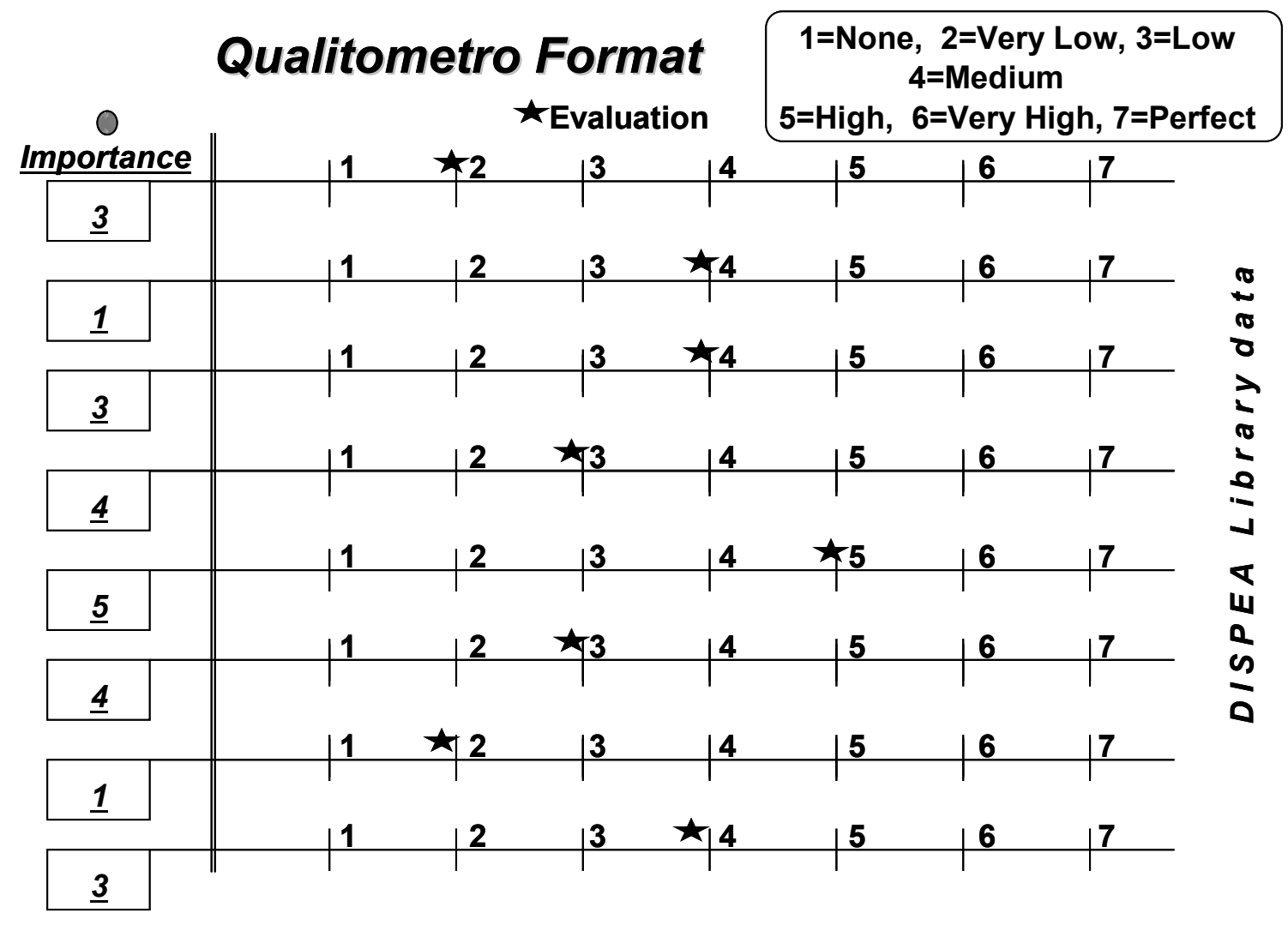

Figure 10. Qualitometro format for collecting data (with Linguistic Variables): actual data 
Grade Of

Membership

Grade of Membership of a Linguistic Variable

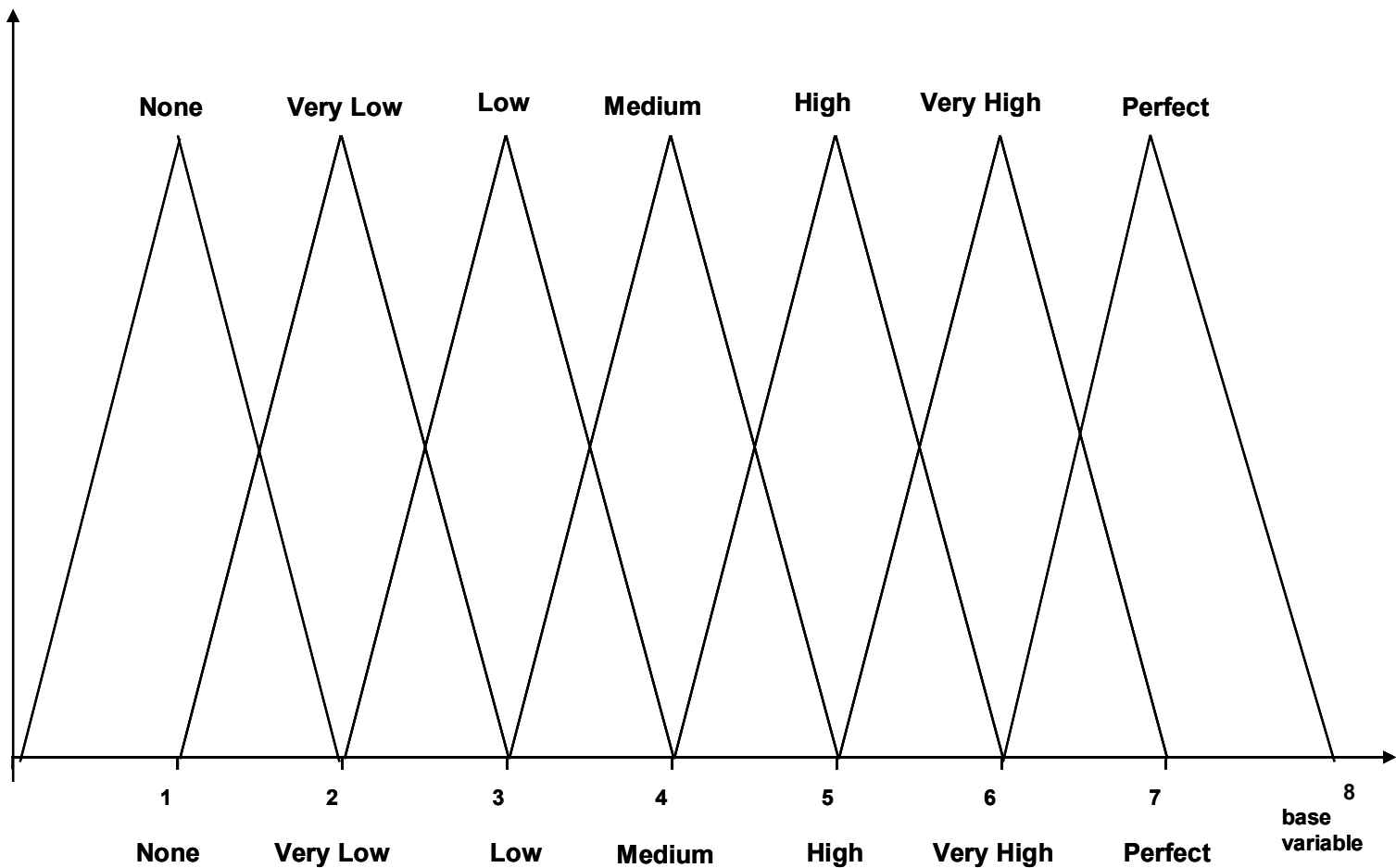

- F. Galetto

Figure 11. Grade of Membership functions for Linguistic Variables [defined by Fausto Galetto]

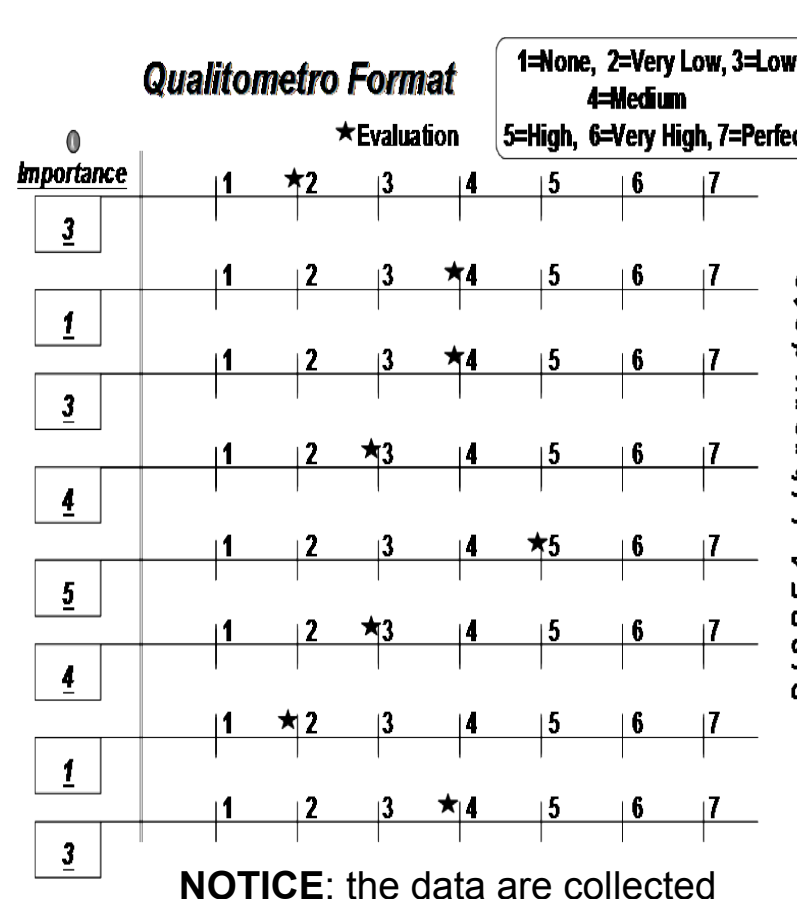

NOTICE: the data are collected as "numbers" in the format
पVh

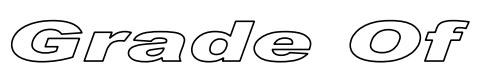

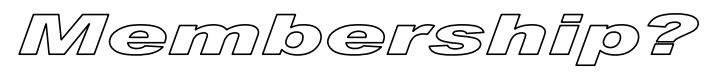

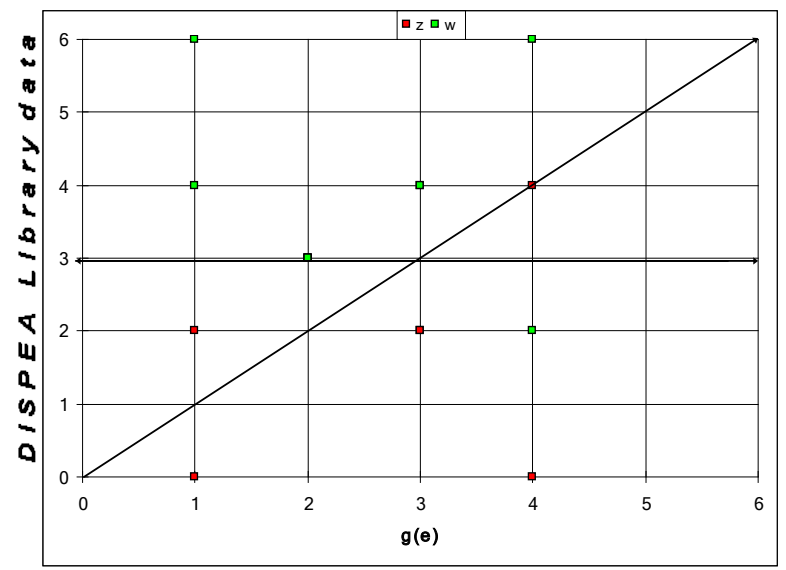

Linguistic data are coded as $0,1,2,3,4,5,6$

[6 is the first "perfect number"]

The criteria are aggregated into a "decision function" q (called "quality" by the service quality evaluators), using fuzzy logic with formula q=Min[Max[Negl(gj), gj(e)]].

Figure 12. Points from the Qualitometro format of figure 10 (format for collecting data left) and NUMERIC Method (right, FG) 
The analysis of the non-numeric data is claimed (by Yager) to be justified using "fuzzy logic": no proof was ever provided. In fact, Yager himself, about his "fundamental" formula $\mathrm{q}=\operatorname{Min}\left[\operatorname{Max}\left[\operatorname{NegI}\left(\mathrm{g}_{\mathrm{j}}\right), \mathrm{g}_{\mathrm{j}}(\mathrm{e})\right]\right]$ says: "... In this section I shall suggest a model that has advantages ..." ... "... we conjecture a general form for this type of decision function" and "... This leads us to conjecture that ..." and eventually "... We can thus see that the proposed model satisfies our intuitive requirements ...".Yager NEVER used the verb "to prove", but only "to suggest", "to conjecture" and "to propose"! It is evident that people claiming that Yager proved (??!!) that formula are in error: for them are very relevant the Deming statements "It is a hazard to copy", "It is necessary to understand the theory of what one wishes to do or to make."

Now it is important to see IF the fuzzy theory is really used in the "Qualitometro method".

Let's consider figure 11, where the GoM [defined by Fausto Galetto] are shown. In order to be really "fuzzy" the Qualitometro should have to use the GoM: therefore we "attach" a GoM to each "value" of the Linguistic Variable, as in figure 11 . We can make all the triangles equal and with area 1; doing that we are able to use Signal Theory and Characteristic Functions (Fourier Transforms of the signal). Apart from a scale shift, the transform of any triangle is $[\sin (\omega / 4) /(\omega / 4)]^{2}$; it is clear that we can have the "fuzzy intersection" of only any two adjacent linguistic values: this intersection has transform $[\sin (\omega / 4) /(\omega / 4)]^{2} / 4$. The square of these transforms is related to the "energy of the signal", that is to the "information content". If we maintain the energy and reduce the "bandwidth of the signals" we arrive at a point where the signals are all apart and there is no intersection; going on this line we arrive at "impulse (Dirac) signals $\delta(\mathrm{x})$ ": the Qualitometro information is nothing but a set of "Dirac signals": so there is nothing fuzzy!!! The limit of $[\sin (\omega / 4) /(\omega / 4)]^{2}$, for $\omega \rightarrow 0$, is the "area under $\delta(x) "$.

In the theory of signals it is proved that $\delta(\mathrm{t})$ is the derivative of $\mathrm{H}(\mathrm{t})$, the Heaviside "unit step signal $[\mathrm{H}(\mathrm{t})=0$ for $\mathrm{t}<0, \mathrm{H}(\mathrm{t})=1$ for $\mathrm{t}>0$ ]". To give sound foundation to this derivative, one needs the "Theory of Distributions" which is a very high Mathematics theory [the theory of Linear Functionals]: therefore the "fuzzy evaluations in the Qualitometro method" are only mathematic Functionals, and fuzzy operations must be defined as operators on Functionals!!! [see any good book of Mathematics]

What is the conclusion from this scientific analysis?

The data in the Qualitometro Format (fig. 10), for each characteristic and for each importance are "unit impulses" (fig. 11 , limit of $[\sin (\omega / 4) /(\omega / 4)]^{2}$, for $\left.\omega \rightarrow 0\right)$ : nothing is fuzzy!

Fuzzy operations (negation, union and intersection) are not defined for "unit impulses"!

Therefore one cannot invoke fuzzy theory as a proof of the formula $\mathrm{q}=\operatorname{Min}\left[\operatorname{Max}\left[\operatorname{NegI}\left(\mathrm{g}_{\mathrm{j}}\right), \quad \mathrm{g}_{\mathrm{j}}(\mathrm{e})\right]\right]$, copied from Yager(see Gell-Mann, fig. 8).

We have learned from the QEG members that «Ordered linguistic scales mainly differ from numeric or ratio scales because the concept of distance is not defined. ... by attributing numbers to verbal information we might effectively [sic] move away from the original logic of the evaluator. In this way any conclusion drawn from the analysis on 'equivalent' numerical data could be partially or wholly distorted.»

We know from Geometry that the refusal of the "parallel axiom" (Euclidean Geometry, see paragraph 2) provided others Geometries (elliptic, and hyperbolic).Here we do the same: we refuse the assumption of "non-scalarisation" and we attribute numbers to verbal information.

Using Logic, the same must happen as it happened for Euclidean Geometry with the refusal of the "parallel axiom": this is the logic and scientific conclusion, from the premises!!!!!!!!!

Since we are fully aware of the validity of the scientific approach, let's apply the ideas given in the section Scientificness: to understand the real validity of Yager Fuzzy Method [copied in the Qualitometro method], we purposely make a scalarization, using the scale $0,1,2,3,4,5,6$ [integer numbers, which form a chain (a very simple lattice)], and using a one-to-one mapping from the linguistic scale [None, Very Low, Low, Medium, High, Very High, Perfect] into the integer numerical scale $[0,1,2,3,4,5,6$ (a chain)], and vice versa, both for the evaluation " $g_{j}(e)$ " to $j^{\text {th }}$ criterion $\left(j^{\text {th }}\right.$ "quality characteristic") and for the importance $I\left(g_{j}\right)$ of each evaluation criterion. Notice: we used 6 the "first perfect number" [Euclid] for the linguistic Perfect, and 0 the "first number" [Peano] for the linguistic None; the number 0 is the least element of the chain, called "zero element" (in Algebra), and the "first perfect number" 6 is the greatest element of the chain, called "one element" (in Algebra), of the set [0, 1, 2, 3, $4,5,6]$.

It is very interesting that any number of the scale is a suitable power of the number 3 "the generator" [for the linguistic Medium] modulo 7 [written shortly mod7]; therefore "Perfect=Medium ${ }^{3}$ modulo 7" Ideas like these were invented more than 2 thousand years ago by Euclid.

The subset $[1,2,3,4,5,6]$ is a finite Abelian group: any element $a$ has one inverse ${ }^{-1}$; the product of two elements is an element of the group (closure); the multiplication is associative; there exists the identity [or one] element. Any integer number is congruent to an element of the set $[0,1,2$, $3,4,5,6]$ : "congruent to" is an equivalent relation (reflexive, symmetric, transitive); there are 7 equivalence classes; equivalence is preserved under addition, subtraction and multiplication. All these properties are well known in Algebra. and were used also by the psychologists Fisch, Watzlawick, Weakland in their book CHANGE .... Other points on Algebra are given later.

We were being using Logic and Algebra [as a $\mu \alpha \theta \eta \tau \eta \varsigma$ ]; let's now use Geometry and Calculus [as a $\mu \alpha \theta \eta \tau \eta \varsigma]$.

Let's consider a plane $\pi$, with Cartesian co-ordinates, [the "Cartesian product" of the two chains (lattices $\mathrm{L}_{1}$ and $\mathrm{L}_{2}$ ) for the importance and for the evaluation is a lattice (L) as well] the couple $(\mathrm{x}, \mathrm{y})$ define a vector OP; the Euclidean norm $\rho$ provides the length of the vector (the distance of the point $P$ 
from the origin $\mathrm{O})$. Therefore the "complete" information provided by an evaluator is given by 8 vectors [ 8 "quality characteristics"], defined by the length and the direction of 8 segments $\mathrm{OP}_{1}, \mathrm{OP}_{2}, \ldots, \mathrm{OP}_{8}$.

We consider then another one-to-one mapping (involution) between the plane $\pi$ (the lattice L, points named $\mathrm{z}$ in fig. 12) and the plane $\pi^{\prime}$ (the lattice $L^{\prime}$, points named $\mathrm{w}$ in fig. 12) defined by the relationship (called "involution") $\mathrm{x}^{\prime}=\mathrm{x}$, $\mathrm{y}^{\prime}=3^{3} \mathrm{y}-1$ modulo 7 ; the 8 vectors $\mathrm{OP}_{1}, \mathrm{OP}_{2}, \ldots, \mathrm{OP}_{8}$ are transformed into 8 vectors $\mathrm{OP}_{1}^{\prime}, \mathrm{OP}_{2}^{\prime}, \ldots, \mathrm{OP}_{8}^{\prime}$. We know from Calculus that for each vector we can define the norm $\left|\mathrm{OP}_{\mathrm{i}}\right|=\max \left(\left|\mathrm{x}_{\mathrm{i}}\right|,\left|\mathrm{y}_{\mathrm{i}}\right|\right)=\left|\mathrm{z}_{\mathrm{i}}\right|$ and obviously $\left|\mathrm{OP}_{\mathrm{i}}^{\prime}\right|=\max \left(\left|\mathrm{x}_{\mathrm{i}}^{\prime}\right|,\left|\mathrm{y}_{\mathrm{i}}^{\prime}\right|\right)=$ $\left|\mathrm{w}_{\mathrm{i}}\right|$; the minimum norm vector is given by formula $\mathrm{q}^{\prime}=$ Min $\left[\left|\mathrm{OP}_{\mathrm{i}}\right|\right]=\operatorname{Min}\left[\left|\mathrm{w}_{\mathrm{i}}\right|\right]$, which provides the aggregated "mapped" result of the Yager Fuzzy Method (and of Qualitometro)

Therefore one [as a $\mu \alpha \theta \eta \tau \eta \zeta]$ gets the same decisionboth if he uses the linguistic scale [N, VL, L, M, H, VH, P] and if he uses the numerical scale $[0,1,2,3,4,5,6]$, with the metric (norm) $\left|\mathrm{OP}_{\mathrm{i}}^{\prime}\right|=\max \left(\left|\mathrm{x}_{\mathrm{i}}^{\prime}\right|,\left|\mathrm{y}_{\mathrm{i}}^{\prime}\right|\right) ! ! ! !$

Let's remind our logic trip[as a $\mu \alpha \theta \eta \tau \eta \varsigma]$ :

1) we used numbers [refusing "non-scalarization" axiom, the important assumption of Qualitometro]

2) we defined a metric (norm) [that does not exists, according the Qualitometro experts!!!!]

3) we used Logic, Algebra, Geometry, Elementary and Higher Calculus [very different ideas from "fuzzy"]

4) we got the same decision as Qualitometro [with very different ideas from "fuzzy"].

The conclusion [for a $\mu \alpha \theta \eta \tau \eta \varsigma$ ] MUST BE logically and scientifically: the statement of the three "old tenors" (of QEG), devisers of Qualitometro "...any conclusion drawn from the analysis on 'equivalent' numerical data could be partially or wholly distorted.» is FALSE. MOREOVER the statement «The problems highlighted above can be alleviated if we resort to analysis tools able to avoid the arbitrary conversion of information. ... The fuzzy operator that is used in the paper allows for this flexibility in the decision logic.» is FALSE.

WHY Fausto Galetto [as a $\mu \alpha \theta \eta \tau \eta \zeta$ ] DOES GET THE SAME RESULT??? All that depends on the isomorphism existing (because we generated it) between the "LINGUISTIC Fuzzy Space" and the "NUMERIC (Algebraic) Space". The linguistic scale [N, VL, L, M, H, $\mathrm{VH}, \mathrm{P}]$ and the integer numeric scale $[0,1,2,3,4,5,6]$ are isomorphic; it is well known (in Algebra) that "An isomorphism between partially ordered sets is an equivalence relation (reflexive, symmetric, transitive)". Any set (of numbers) $\left[\mathrm{y}_{0}, \mathrm{y}_{1}, \mathrm{y}_{2}, \mathrm{y}_{3}, \mathrm{y}_{4}, \mathrm{y}_{5}, \mathrm{y}_{6}\right]$ isomorphic to the scale [N, VL, L, M, H, VH, P] does well!!!!

We [as a $\mu \alpha \theta \eta \tau \eta s]$ purposely made what was forbidden and made our worst use of: numerical scales, scalarization, arbitrary and artificial conversion of linguistic collected data, distance and metric concepts, non-fuzzy logic and operators, $\ldots$ and we found the shocking result:

we got the same quality evaluation:

all those "fuzzy" requirements are not essential.

The papers of QEG and Yager were published in scientific magazines and were praised by the referees and the editors (by publishing them); therefore are considered scientific by the scientific community: they are cited and have high impact points.!!!!Are they good papers????

In this paper we [as a $\mu \alpha \theta \eta \tau \eta \zeta$ ] proved that those papers comprise FALSE STATEMENTS.How can be considered scientific a paper with false statements (even though it is cited many times)?

There are several proofs of falsenesses in the Qualitometro; Fausto Galetto[as a $\mu \alpha \theta \eta \tau \eta \zeta]$ provided a completely numeric (algebraic) way. For each "quality characteristics" we compute the number

$$
\mathrm{w}_{\mathrm{i}} \quad=\left[\left(\left(\mathrm{x}_{\mathrm{i}}+3^{3} \mathrm{y}_{\mathrm{i}}-1\right.\right.\right.
$$

$\bmod 7)) / 14-\operatorname{INT}\left(\left(\left(x_{i}+3^{3} y_{i}-1\right.\right.\right.$

$\bmod 7)) / 14))]^{* 7}$

and thenmin $\left(\mathrm{w}_{\mathrm{i}}\right)$ provides the number that, when it is anti-transformed, gives the "Fuzzy Result"!!!!!!!!!!

All that depends on the isomorphism existing (because we [as a $\mu \alpha \theta \eta \tau \eta \zeta$ ] generated it) between the "LINGUISTIC (non-Fuzzy) Space" and the "NUMERIC (Algebraic) Space".

The linguistic scale [None, Very Low, Low, Medium, High, Very High, Perfect] and the integer numerical scale $[0$, $1,2,3,4,5,6]$ are isomorphic; it is well known (in Algebra) that "An isomorphism between partially ordered sets is an equivalence relation (reflexive, symmetric, transitive)".

At this point, there should be no doubt, to any "Intellectually Honest" person, that the statement "Scalarization, introducing in the scale ... some "metrological properties" ... may determine a "distortion" effect on information meaning ... introducing through coding an arbitrary metric, resulting in a wrong interpretation of gathered data. ..." is false.

Scalarization does not introduce "distortion". Logic says that.

One can think that the mapping (isomorphism) into the numerical scale $[0,1,2,3,4,5,6]$ was only a lucky choice: there are infinite mappings, due to isomorphism (which is an equivalence relation) of any finite chains with the same number of elements; two of them are $[1,2.5,4,8.5,17.5$, $35.5,71.5],[1,3.5,6,13.5,27.5,57.5,116.5]$. Also the "Magic Numbers" [2, 8, 20, 28, 50, 82, 126] could have been used; a very intriguing set is, as well, the "Fibonacci Numbers". Obviously the involution is not defined by the relationship given before: in any case, a suitable transformation exists and is not reported here.

We could stop here, mentioning only that there are other several proofs of the falseness of the statements related to the Yager (Linguistic) Fuzzy Method and to Qualitometro. No "fuzzy" concepts are EVER used! The interested reader [as a $\mu \alpha \theta \eta \tau \eta \varsigma]$ shall contact F. Galetto.

Please see the part where the Qualitometro III is presented: the same QEG provides a new version where noting of "fuzzy"is present. (it was published in the magazine "Quality and Reliability Engineering International"). There, the three "new tenors" give the lie to their "false" previous statements: fuzzy logic is useless. 
Remember that a fuzzy set $A$, is an "ordered" couple [X, $\mathrm{GoM}]$ characterized by associating with each object $x$ in a universe of discourse $X$ (or "base variable" $x$ ) a value, called Grade of Membership (GoM) of $x$ in A, which is a number varying between 1 (i.e. full membership) and 0 (i.e. nonmembership); therefore a fuzzy set $A$ is a set of a Cartesian plane, so that in abscissa we have the element $x$ and in ordinate the corresponding GoM, also called membership function $\mu_{A}(\mathrm{x}): X \rightarrow[0,1]$, which associates to each $x$ of $X$ a number $\mu_{A}(x)$ in the interval $[0,1]$, that represents just the GoM of $x$ in $A$; it means that, actually, the fuzzy set $A$ is a set of ordered pairs, the first element being $x$ and the other the corresponding GoM, which is a function in the mathematical sense: one defines "fuzzy sets" through the "normal" concepts learned in Mathematics.

Universities will not improve IF their professors (as well the referees) are not able to see the myriads of errors given to students by "distinguished colleagues, whose achievements and academic standing are well above ...".

Professors teaching in university courses, when they are found in error (non-conformity) [see Quality ideas], MUST consider that, for Quality, any non-conformity must generate the search of its causes and then implementation of Corrective Actions. Professors were no good, because they did not take Preventive Actions ... At least they should have taken Corrective Actions. Why professors act that way?

We present now the "new" ideas of the QEG in the paper "Ordered Samples Control Charts for Ordinal Variables" published in Quality and Reliability Engineering International [named by my students "the Qualitometro III Method"].

The new three "tenors" say «The paper presents a new method for statistical process control when ordinal variables are involved. This is the case of a quality characteristic evaluated by on ordinal scale. The method allows a statistical analysis without exploiting an arbitrary numerical conversion of scale levels and without using the traditional sample synthesis operators (sample mean and variance). It consist of different approach based on the use of a new sample scale obtained by ordering the original variable sample space according to some specific 'dominance criteria' fixed on the basis of the monitored process characteristics. Samples are directly reported on the chart and no distributional shape is assumed for the population (universe) of evaluations".»

As usual (as done in Qualitometro I and in Qualitometro II) the new three "tenors" add: «Many quality characteristics are evaluated on linguistic or ordinal scales... ... The levels of this scale are terms such as 'good', 'bad', 'medium', etc..., which can be ordered according to the specific meaning of the quality characteristic at hand. Ordered linguistic scales mainly differ from numerical or cardinal scales because the concept of distance is not defined. The ordered is the main property associated to such scales. ... The problem of on-line monitoring of ordinal quality characteristic required the development of techniques able to deal with ordinal data. The assignment of weights, demerits and so on, to reflect the degree of severity of product non-conformity, has been adopted in many circumstances. Different numbers of the demerits are assigned to each class and the total number of demerits is monitored by some control chart for defectives. This is a subjective approach that requires the ability to uniquely classify each state into of several mutually exclusive classes, with well-defined boundaries among them. » ... If one decides to use numbers two problems arise (as in Qualitometro I and as in Qualitometro II): «the first is concerned with the validity of encoding a discrete verbal scale into a numerical form. The numerical codification implies fixing the distances among scales levels, thus converting the ordinal scale into a cardinal one; the second is related to the absence of consistent criteria for the selection of the type of numerical conversion. It is obvious that changing the numerical encoding may determine a change in the obtained results. In this way the analyst directly influences the acceptance of results. Therefore, any conclusions drawn from the analysis on 'equivalent' numerical data could be partially or wholly distorted» (as in Qualitometro I and in Qualitometro II).

Then they present the cork example, where thy classify the "quality" of the cork as 'reject' if the cork does not work; 'poor quality' if the cork must not be rejected but has some defects; 'medium quality' if the cork has relevant aesthetic flaws but no other defects; 'good quality' if the cork only has small aesthetic flaws; 'excellent quality' if the cork is perfect. 30 data are collected; they are in table 1

Table 1. Data collected on corks

\begin{tabular}{|c|c|c|c|c|}
\hline Reject & Poor quality & Medium q. & Good q. & Excellent quality \\
\hline 2 corks & 5 corks & 9 corks & 7 corks & 7 corks \\
\hline
\end{tabular}

If one codify as follows the "quality" 'reject'=1; 'poor quality' $=2$; 'medium quality' $=3$; 'good quality' $=4$; 'excellent quality' $=5$, the "average quality" is 3.4 ; «Hence, the sample mean seems to be between 'medium quality' and 'good quality'. The adopted numerical conversion is based on the implicit assumption that all scale levels are equispaced. However, we are not sure that the evaluator perceives the subsequent levels of the scales as equispaced, nor even if s/he has been preliminary trained». If one codify as follows the "quality": 'reject' $=1$; 'poor quality'=3; 'medium quality'=9; 'good quality'=27; 'excellent quality' $=81$ the "average quality" is 28.5 ; «The sample mean seems to be between 'good quality' and 'excellent quality'. We cannot say which the right value of the sample mean is at hand because an 'exact' codification does not exist... ...This example points out that a simple codification of scale levels could result in a misrepresentation of the original gathered information. A correct approach should be based on the usage of the properties of ordinal scales themselves. The main aim of the present paper[Qualitometro III] is to propose a new method for on-line process control of a quality characteristic evaluates on an ordinal scale, without exploiting an artificial conversion of scale levels... ...The 
new proposal does not consider the synthesis operators. It allows on-line monitoring based on a new process sample scale obtained by ordering the original variable sample space according to some specific 'dominance criteria'. Samples are directly reported on the chart and no distributional shape assumed for the population (universe) of evaluations».

From this example, it is apparent that "codification" affects the resulting "average quality".

To avoid this problem the new three "tenors" of QEG introduce the sample space and the dominance criteria. They say: «The sample space of a generic ordinal quality characteristic is not ordered in nature».«A dominance criterion allows attributing a position in the ordered sample space to each sample. If sample $B$ dominates sample $A$, the sample $A$ has a lower position in the ordering. For each pair samples a dominance criterion states a dominance or an equivalence relationship. If the resolution of the dominance criterion is high, the dimension of equivalence classes is very small. The most resolving criterion is the one assigning a different position to each ordered sample. This is the same as saying that every equivalence class has only one element».

They provide the following example, where 10 samples (of 4 items) are checked and their "quality" is rated as: 'high' $\rightarrow \mathrm{H}$; 'medium' $\rightarrow \mathrm{M}$; 'low' $\rightarrow \mathrm{L}$. Then they say: «The first classical answer to this question is the assignment of a specific numerical value to each level of the evaluation scale. A possible codification could be the following: 'Low' $=1$; 'Medium'=2; 'High'=3», giving the right part of the table, with the means and the ranges

The grand mean is $\bar{x}=2.45$, and the mean Range is $\bar{R}=1$. Then the "classic" control limits are $\mathrm{LCL}_{\mathrm{x}}=1.721$, $\mathrm{UCL}_{\mathrm{x}}=3.179$ and $\mathrm{LCL}_{\mathrm{R}}=0, \mathrm{UCL}_{\mathrm{R}}=2.282$

«The codification allows building traditional $\bar{X}-R$ control charts. However, as anticipated, this procedure has three main contraindications. First, each conversion is arbitrary and different codifications can lead to different results. Second, codification introduces the concept of distance among scale levels, which is not originally defined. Third, since the original distribution of evaluations is discrete with a very small number of levels, the central limit theorem hardly applies to this context. A second analysis of data in Table 2 can be executed by method suggested by the QEG members. This methodology is based on the use of operators that do not require the numerical codification of ordinal scale levels. The adopted location measure is the ordered weighted average (OWA) emulator of arithmetic mean, firstly introduced by Yager and File». [it is Qualitometro II] «The OWA operator [it is Qualitometro II] can take values only in the set of levels of the original ordinal scale. The related control chart is built following a methodology very similar to the traditional chart for mean values. The adopted dispersion measure is the range of ranks $r_{s}$, defined as the total number of levels contained between the maximum and the minimum value of a sample (the rank $r(q)$ is the sequential integer number of a generic level $q$ on a linguistic scale): $r_{s}=\left[r(q)_{\max }-r(q)_{\min }\right]$. For the range of ranks too, the related control chart is constructed using the traditional approach. Figures V.3 and V.4 show the control charts for the OWA and the range of ranks of data reported in Table 2». The Control Limits are [it is Qualitometro II, left of table 2]:

$$
\begin{gathered}
U C L_{\bar{q}}=\overline{\bar{q}} \oplus \operatorname{Int}(U * \bar{r})=H \\
U C L_{r}=C L_{r} \oplus \operatorname{Int}\left(V_{1} * \bar{r}\right)=2 \\
C L_{\bar{q}}=\overline{\bar{q}}=M \\
C L_{r}=\operatorname{Int}(\bar{r})=1 \\
L C L_{\bar{q}}=\overline{\bar{q}} \Theta \operatorname{Int}\left(U^{*} \bar{r}\right)=L \\
L C L_{r}=C L_{r} \Theta \operatorname{Int}\left(V_{2} * \bar{r}\right)=0
\end{gathered}
$$

The new three "tenors" of the QEG add: «Although this methodology does not exploit the device of codification, the dynamics of the charts are poor and little information can be extracted about the process [while BEFORE for Qualitometro II they said, some years before, that it was fantastic!!!!]. Moreover, the method is not free from distributional assumptions [while BEFORE for Qualitometro II they said, some years before, that it was distribution free!!!!]. The dispersion measure assumes that the scale ranks do not depend on the position of level of the ordinal variable. In this paper [Qualitometro III] we propose a third way of analysing data reported in Table 1. It exploits the only properties of ordinal scales, avoiding the synthesis of information contained in the sample. No distributional assumptions are required about the population (universe) of evaluations. As traditional control charts, this new methodology is based on the use of two different charts: one for ordered sample values, and the other for ordered sample ranges... ...As a consequence, they can be built and used separately. However, for an exhaustive analysis, a conjoint approach is highly recommended.

"The new proposal is based on the ordering of the sample space of the ordinal quality characteristic». They show the example of 3 samples A, B, C, of 3 items (with codification High, Mean, Low): sample A: $\{H, M, M\}$; sample B: $\{H, H, L\}$; sample C: $\{M, M, M\}$.

«To compare and order these samples we introduce a rule called 'dominance criterion', defined, case by case, on the basis of the characteristics of the monitored process. In accordance with this rule, if sample A dominates sample B, then sample A is preferred to sample B. As a result we can define a new ordinal scale whose levels are the positions of the samples in the ordered sample space. If there is no dominance relationship between sample A and sample B, they belong to the same 'equivalence class'. The choice of the dominance criterion influences the resolution of the scale (i.e. the number of levels of the ordered sample space) and also the order of levels. For each process one or more dominance criterion may be established on the basic of the specific application».

The new three "tenors" of the QEG say:«We begin 
analysing the Pareto-dominance criterion. We state that sample X Pareto-dominates sample $\mathrm{Y}$ if all elements in $\mathrm{Y}$ do not exceed the corresponding elements in $\mathrm{X}$, and at least one element in $\mathrm{X}$ exceeds the corresponding one in $\mathrm{Y}$. This situation is formally denoted by $X \triangleright Y$. In case samples $\mathrm{X}$ and $\mathrm{Y}$ belong to the same equivalence class, i.e. no dominance relationship can be defined between them, we use the following notation: $X \approx Y$ ". For the example they find $A \triangleright C ; A \approx B ; B \approx C$.

As we can see from figure [not given here], it is not possible to assign a well-defined position to samples A, B and $\mathrm{C}$, because their intersection is not empty. The problem can be solved by introducing the concept of 'semi-equivalence class'. A semi equivalence class is composed of equivalence classes whose intersections are not empty...»

The new three "tenors" add: In general, Pareto dominance criterion gives a 'poor' ordering for the sample space of an ordinal quality characteristic. A more discerning criterion is the 'rank dominance criterion'. Its introduction requires the definition of the concept of 'optimal sample'. A sample is said to be optimal if all elements assume the highest level of an ordinal scale. In our example the optimal sample is HHH. For each sample we define a rank index which quantifies its positioning with regard to the optimal sample. The index is built in by adding up the numbers [notice: numbers] of scale levels contained between each sample value and the corresponding value of the optimal sample».

Table 2. Data on the quality of 40 items [ 10 samples of 4] and their "scalarization" with mean and range (right hand).

\begin{tabular}{|c|c|c|c|c|c|c|c|c|c|c|c|c|c|}
\hline & \multicolumn{5}{|c|}{ Data } & mean & $\mathrm{R}$ & \multicolumn{5}{|c|}{ data } & \multicolumn{2}{|c|}{ mean } & $\mathrm{R}$ \\
\hline 1 & $\mathrm{H}$ & $\mathrm{H}$ & $\mathrm{M}$ & $\mathrm{H}$ & $\mathrm{M}$ & 1 & 1 & 3 & 3 & 2 & 3 & 2.75 & 1 \\
\hline 2 & $\mathrm{H}$ & $\mathrm{M}$ & $\mathrm{H}$ & $\mathrm{H}$ & $\mathrm{M}$ & 1 & 2 & 3 & 2 & 3 & 3 & 2.75 & 1 \\
\hline 3 & $\mathrm{H}$ & $\mathrm{M}$ & $\mathrm{M}$ & $\mathrm{H}$ & $\mathrm{H}$ & 1 & 3 & 3 & 2 & 2 & 3 & 2.50 & 1 \\
\hline 4 & $\mathrm{H}$ & $\mathrm{H}$ & $\mathrm{M}$ & $\mathrm{L}$ & $\mathrm{M}$ & 2 & 4 & 3 & 3 & 2 & 1 & 2.25 & 2 \\
\hline 5 & $\mathrm{H}$ & $\mathrm{M}$ & $\mathrm{M}$ & $\mathrm{H}$ & $\mathrm{H}$ & 1 & 5 & 3 & 2 & 2 & 3 & 2.50 & 1 \\
\hline 6 & $\mathrm{M}$ & $\mathrm{H}$ & $\mathrm{M}$ & $\mathrm{M}$ & $\mathrm{M}$ & 1 & 6 & 2 & 3 & 2 & 2 & 2.25 & 1 \\
\hline 7 & $\mathrm{H}$ & $\mathrm{M}$ & $\mathrm{H}$ & $\mathrm{M}$ & $\mathrm{M}$ & 1 & 7 & 3 & 2 & 3 & 2 & 2.50 & 1 \\
\hline 8 & $\mathrm{~L}$ & $\mathrm{~L}$ & $\mathrm{M}$ & $\mathrm{L}$ & $\mathrm{M}$ & 1 & 8 & 1 & 1 & 2 & 1 & 1.25 & 1 \\
\hline 9 & $\mathrm{M}$ & $\mathrm{H}$ & $\mathrm{H}$ & $\mathrm{H}$ & $\mathrm{H}$ & 1 & 9 & 2 & 3 & 3 & 3 & 2.75 & 1 \\
\hline 10 & $\mathrm{H}$ & $\mathrm{H}$ & $\mathrm{H}$ & $\mathrm{H}$ & $\mathrm{H}$ & 0 & 10 & 3 & 3 & 3 & 3 & 3.00 & 0 \\
\hline
\end{tabular}


It is important to notice the statement "a rank index which quantifies" the distance between any sample and the optimal sample!!! This is a distance defined on sets (see any good book on Calculus). In spite of that, the new three "tenors" of the QEG were continuing to say "the concept of distance is not defined"!!!!!!!!!!!!

Table 3. ordered samples for a sample space defined by a 3-level scale ( $\mathrm{L}=$ Low; $\mathrm{M}=$ Medium; $\mathrm{H}=$ High) and a sample size $\mathrm{n}=4$. Column 3 reports the positions of each sample after the application of the rank dominance criterion. Column 4 shows the position of each sample after the sequential application of the rank and the dispersion dominance criteria.

\begin{tabular}{|c|c|c|c|}
\hline & $\begin{array}{c}\text { Pareto-dominance } \\
\text { criterion }\end{array}$ & $\begin{array}{c}\text { rank } \\
\text { dominance } \\
\text { criterion }\end{array}$ & $\begin{array}{l}\text { rank dominance } \\
\text { criterion with the } \\
\text { dispersion } \\
\text { dominance } \\
\text { criterion }\end{array}$ \\
\hline $\begin{array}{l}\text { Sample } \\
\text { Space }\end{array}$ & Rank Index & $\begin{array}{c}\text { Position in } \\
\text { the ordered } \\
\text { sample space } \\
\text { (equivalent } \\
\text { class) } \\
\text { [rank } \\
\text { dominance } \\
\text { criterion] }\end{array}$ & $\begin{array}{c}\text { Position in the } \\
\text { ordered sample } \\
\text { space(equivalent } \\
\text { class) } \\
\text { [rank and } \\
\text { dispersion } \\
\text { dominance } \\
\text { criterion] } \\
\end{array}$ \\
\hline LLLL & 8 & $1^{\text {st }}$ & $1^{\text {st }}$ \\
\hline MLLL & 7 & $2^{\text {nd }}$ & $2^{\text {nd }}$ \\
\hline MMLL & 6 & $3^{\text {rd }}$ & $4^{\text {th }}$ \\
\hline MMML & 5 & $4^{\text {th }}$ & $6^{\text {th }}$ \\
\hline MMMM & 4 & $5^{\text {th }}$ & $9^{\text {th }}$ \\
\hline HLLL & 6 & $3^{\text {rd }}$ & $3^{\text {rd }}$ \\
\hline HMLL & 5 & $4^{\text {th }}$ & $5^{\text {th }}$ \\
\hline HMML & 4 & $5^{\text {th }}$ & $8^{\text {th }}$ \\
\hline HMMM & 3 & $6^{\text {th }}$ & $11^{\text {th }}$ \\
\hline HHLL & 4 & $5^{\text {th }}$ & $7^{\text {th }}$ \\
\hline HHML & 3 & $6^{\text {th }}$ & $10^{\text {th }}$ \\
\hline HHMM & 2 & $7^{\text {th }}$ & $13^{\text {th }}$ \\
\hline HHHL & 2 & $7^{\text {th }}$ & $12^{\text {th }}$ \\
\hline HНHM & 1 & $8^{\text {th }}$ & $14^{\text {th }}$ \\
\hline НHНH & 0 & $9^{\text {th }}$ & $15^{\text {th }}$ \\
\hline
\end{tabular}

«A high value of rank index corresponds to a 'bad' sample. All samples that are characterized by the same index belong to the same equivalence class. Therefore their positioning with respect to the optimal sample can be equivalently identified by the corresponding equivalence class. The number of elements of the new ordinal sample scale depends on the sample size and on the number of levels of the evaluation scale. Denoting by $\mathrm{t}$ the number of levels of the evaluation scale and by $\mathrm{n}$ the sample size, the rank dominance criterion gives a number of equivalence classes equal to $n(t-1)+1$. This is also the number of levels of the resulting ordinal scale of sample positions». "Table 2 (first column) reports all possible ordered samples of size $n=4$, on an evaluation scale with $\mathrm{t}=3$ levels. For each sample, the corresponding position on the resulting scales is reported (third column). The greater the position number, the higher the sample evaluation. A greater resolution, i.e. a larger number of levels, on the ordinal sample scale can be obtained by integrating the rank dominance criterion with the dispersion dominance criterion. This criterion allows distinguishing among samples belonging to the same equivalence class by analysing sample dispersion. A lower position is associated with a greater dispersion. The fourth column of table 2 reports the position of each sample in the new ordered sample space after the sequential application of the rank and the dispersion dominance criteria. As we can see, each ordered sample is associated with a different position; this is the greatest possible resolution».

«The three dominance criteria introduced are consistent. A richer dominance dispersion splits the equivalence or semi-equivalence classes given by the poorer criteria, refining the order of the sample space».

«The resolution of the ordered sample space varies with the considered dominance criterion. In accordance with a specific dominance criterion, sample charts report the positions of samples in the ordered sample space on the vertical axis. Given the particular meaning of sample charts, only the lower control limit (LCL) is defined. The central line $(\mathrm{CL})$ represents the median of sample distribution. A set of initial samples is considered to determine the sample empirical frequency distribution. This empirical distribution[then a distribution exists!!! We shall see that it is the multinomial!!!] is then used to calculate the lower control limit for a given type I error. Control limits are determined by empirical estimates of probabilities[not taking into account the confidence interval!!!] based on observed frequencies in a set of initial samples. Therefore, because the probabilities are estimated, the estimates contain errors, which could become significant for very small probabilities. A large initial set of samples or an alternative approach based on bootstrap techniques are needed to estimate the limits with a more reasonable accuracy» [not necessary BECAUSE a distribution exists: the multinomial!!!].

Let's consider, again, samples of size 4; if each item is classified in 3 possible ways (L, M, H), the possible ordered samples (permutations with repetitions) are 81 . If the order, within each sample, makes no difference [e.g. MMHL is not different from HMLM, (combination without repetitions), and so on], the possible states reduce to 15 . The application of the three criteria provides the positions in table 3 . For the collected data (table 2) The new three "tenors" of the QEG, find «Ordered sample charts are rich insights from different points of view. The resolution of charts increases while shifting from case Pareto dominance criterion to rank dominance criterion to rank and dispersion dominance criterion. The information depicted in the charts is strictly connected: this can be observed on analysing peaks and valleys in the same position. Furthermore, comparing these results with those obtained in this figure 1 [not given here], 
some differences appear. .... It must be noted that the two approaches give, in the example, very similar results because the adopted ordering criteria (applied to ordinal scales) have the same 'monotonic' properties as the mean operator (arbitrarily) adopted a linear codification of levels. With different codifications and criteria the difference between the proposed approach and the tradition, al one would be more marked».

We show now that we can assign numbers to any classification of the items (products, services, ...) checked... and we get the same results as the "dominance criteria" [of the new three "tenors"]: numerisation provides NO distortion.

Using the codification 'Low' $=0$; 'Medium' = 1 ; 'High' = 2 , one finds the results of the "rank dominance criterion". [same distance between scale values]. Using the codification 'Low' = 2.5; 'Medium' = 1; 'High' = 0, one finds the results of the "rank and dispersion dominance criterion". [different distance between scale values]

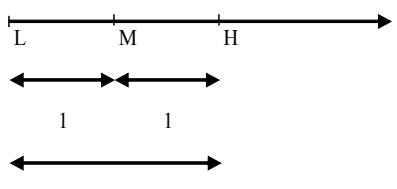

2

Using the numbers we get the same results as the "dominance criteria" [of the new three "tenors" of the QEG]!!!!!!!!!

If one "scalarises" the data as $L=2.5, M=1, H=0$, he finds the same 15 "classes of equivalence" as those of the "rank and dispersion dominance criterion". If one uses the multinomial,

$f_{X_{1}, X_{2}, \ldots, X_{k}}\left(x_{1}, x_{2}, \ldots, x_{k}\right)=\frac{n !}{x_{1} ! x_{2} ! \ldots x_{k+1} !} p_{1}^{x_{1}} p_{2}^{x_{2}} \ldots p_{k+1}^{x_{k+1}}$, he can find the probability of each "class of equivalence": that allows the calculation of the UCL and LCL of the control charts. You get the same results if you use, as well, either $L=15, M=10, H=4$ or $L=4, M=10, H=15$; try and see!!!!

Is there any doubt that one can use numbers and that there is a distribution connected with the sampling?

Surely there is no doubt that

- «The numerical codification implies fixing the distances among scales levels, thus converting the ordinal scale into a cardinal one; the second is related to the absence of consistent criteria for the selection of the type of numerical conversion [are consistent the "dominance criteria" of the new three "tenors"?????]. It is obvious that changing the numerical encoding [as the "dominance criteria" of the new three "tenors"] may determine a change in the obtained results. In this way the analyst directly influences the acceptance of results».

- «Therefore, any conclusions drawn from the analysis on 'equivalent' numerical[as the "dominance criteria" of the new three "tenors" of the QEG] data could be partially or wholly distorted»
- «By the adopted criteria, the example presents some significant differences compared with the approach based on the numeric codification of levels. Using different criteria the difference between the proposed approach and the traditional one becomes more evident, such as for the ordinal sample charts. Furthermore, ordinal range charts also allow a process positioning analysis. A good quality process will present a concentration of samples at the lowest positions of the ordinal range space scale».

- «The paper presents two new control charts for the process control of quality characteristics evaluated on an ordinal scale, without exploiting an artificial conversion of scale levels. The basic concept of the charts is the ordering of the sample space of the quality characteristic at hand».

- «Charts do not suffer from the poor resolution shown by other linguistic charts [while BEFORE for Qualitometro II they, members of the QEG, said, some years before, that they were fantastic!!!!], where the original evaluations scale is used to evaluate samples».

- «No distributional shape is assumed for the population (universe) of evaluations». [a distribution exists!!! it is the multinomial!!!]

\section{are FALSE statements !!!!!!!!!!!!!!!!!}

Only the incompetent people do not understand that. A question is eventually important: why, for the same data, the "Valdese Hospital", collected by the QEG, the importance of criteria was very important in Qualitometro II, while was no longer important in Qualitometro III??????????????

IF these papers of the QEG were cited by many other papers, would that mean that the QEG authors are good?

\section{Logistics and Inventory}

We have seen that papers cited and published on "good" magazinies sometimes are very poor in their scientificness standing. Now we will see that also books, used in universities, have the same problems. We consider now the case of inventories, as given in Brandimarte, P., Zotteri G. and Hopp W, J, Spearman M, L. This case can be related to "preventive replacement before out-of-stock". See section 1.

Carefully managing inventory levels makes good economic sense: it improve customer service and reduce costs.Inventory plays a key role in the logistical behaviour of all manufacturing systems. The classical inventory results are central to more modern techniques of manufacturing management, such as material requirement planning (MRP), just-in-time (JIT) and time based competition (TBC).

If delivery times are known and fixed (lead time $\mathrm{LT}=0$ means immediate delivery) the Wilson formula is valid $Q=\sqrt{2 A \lambda / h}$.

If delivery times are uncertain, as "random variables" are, then a different approach is required. There is, however, a more important source of randomness: the demand. In this 
case we enter the "Statistical Reorder Point" model developed by Wilson (1934). Probability concepts are needed because demand is random. The model is named $(\mathrm{Q}$, $\mathrm{R})$ model and works this way: inventory is monitored continuously; when the inventory level reaches (or goes below) R, an order of size Q is placed; after a lead time LT, during which a stockout might occur, the order is received: the problem is to determine appropriate values of $\mathrm{Q}$ and $\mathrm{R}$. Now the demand $\mathrm{X}$ (in units) is a random variable; for convenience it is considered as continuous, with cumulative distribution $\mathrm{F}(\mathrm{x})[\mathrm{CD}]$ and probability density function $\mathrm{f}(\mathrm{x})$ [pdf]: $\mu$ is the mean demand and $\sigma$ is the standard deviation of demand. The replenishment lead time LT is assumed constant. Since we place an order when there are $\mathrm{R}$ units in stock and we expect to incur demand while we wait for the replenishment order to arrive, we face the case where we can go out-of-stock: we do not have any more units to sell, we are in stockout. Two cases can happen causing bad Service Level and consequent losses: either customers are willing to wait until the order arrives [we name it "back-order" model, or "type II Service" model], or customers do not wait and buy the product from another supplier [we name it "stockout" model, or "type I Service" model]. For both the models it is important to consider the IP (Inventory Position) which takes into account the on-hand inventory (the physical inventory in stock) the backorders and the replenishment orders: $\mathrm{IP}=$ on-hand inventory - backorders + orders. A replenishment order for quantity Q is placed any time the IP becomes $\leq \mathrm{R}$; after a constant $\mathrm{LT}$ the order is received: unfortunately, in the meantime a stockout might occur.

To show F. Galetto ideas we consider here the "stockout" or "type I Service" model, as he found it in books and papers: "If stockouts are permitted and lead time is $L T>0$, if demand is random, the formula to be minimised is the $\mathrm{A} \lambda / \mathrm{Q}+\mathrm{h}(\mathrm{R}+\mathrm{Q} / 2-\lambda \mathrm{LT})+\left(1-\mathrm{F}_{\mathrm{LT}}(R)\right) \mathrm{p} \lambda / \mathrm{Q}$ (where $R$ is the trigger quantity for launching the order $Q, p$ is the penalty for stockouts and $F_{L T}(d)$ is the probability distribution of the demand d, during the lead time $L T^{\prime \prime}$. Actually the formula is inconsistent [see section 1].

No scientific proof of the formula was ever provided!!!

Understanding that the formula is wrong is very easy.

The probability of stockout depends on the competition of two stochastic processes: the demand versus the replenishment.Actually the formula $\mathrm{A} \lambda / \mathrm{Q}+\mathrm{h}(\mathrm{R}+\mathrm{Q} / 2-\lambda \mathrm{LT})+\left(1-\mathrm{F}_{\mathrm{LT}}(R)\right) \mathrm{p} \lambda / \mathrm{Q}$ is inconsistent, because it is based on an intuitive [not proved] extension of the caseof $<<$ "constant" demand, no stockouts permitted and lead time zero $>>$.

To grasp the essential features of the $(\mathrm{Q}, \mathrm{R})$ model, one can make a Gedanken Experiment, using the Bernoulli's Theorem (equation of motion in hydrodynamics) to guess: let's consider a physical system of three water containers with the same base area, as in the figure 13; the water flows from the discharge tube of the middle container; when the water level of the middle container falls below R level, the pump is triggered and the water flows (from the lower container) into the upper container till the level Q is achieved: at this time the water is immediately dropped into the middle container. IF this happens before the 0 level in the middle container is reached, the probability of water "stockout" is zero! One needs many cycles to experience water "stockout"!!!! [depending on the random factors, pump delivery and discharge tube, in order to take into account the random demand and the random replenishment time].

The formula does not "agree" with figure 13, and therefore is wrong, even though I did not provide the right one: you can get all that using F. Galetto ideas, as given in his books and papers.

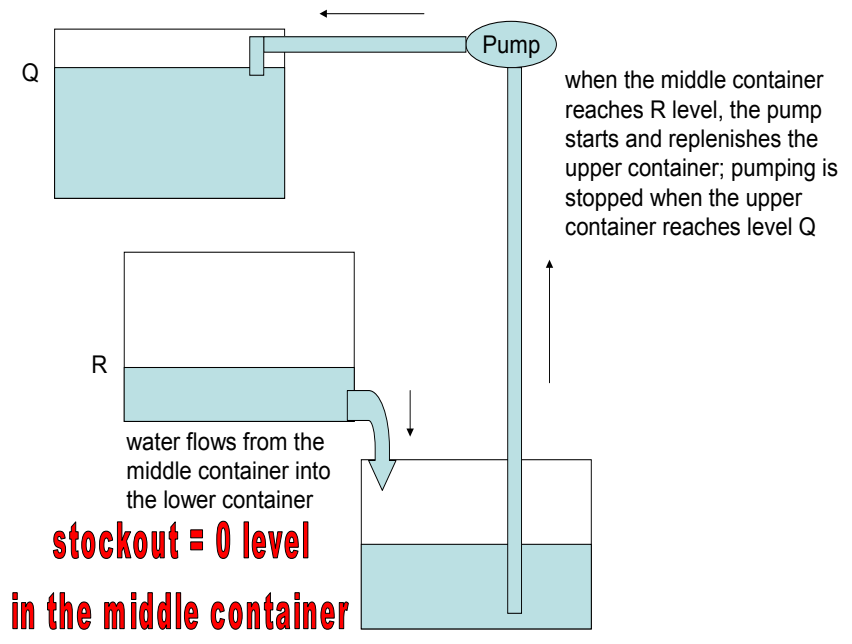

Figure 13. the water tanks

In the following pages we will consider several examples, with wrong solutions, from various sources[but the authors have high scores].We give here some to grasp immediately the critical situation in various universities.

For example Brandimarte and Zotteri, in their book, found $\mathrm{Q}=111, \mathrm{R}=143$ and type I Service level $=95.73 \%$, using the following data: $\mathrm{A}=50, \mathrm{~h}=2, \mathrm{p}=500$, Normal Distribution during $\mathrm{LT}=6$ months, with pdf $\mathrm{N}(\mu, \sigma)=\mathrm{N}(100,25)$.

Using the same data, and the right ideas, F. Galetto finds $\mathrm{Q}=80, \mathrm{R}=92$ and type I Service level=95.73\%: lower costs for the same service level!!!! This result comes from the right formulae.

As another example Hoop and Spearman, in their book Factory Physics, say "Jack, the maintenance manager, has collected historical data that indicate one of the replacement parts he stocks has annual demand of 14 units per year.", and find $\mathrm{Q}=4, \mathrm{R}=3$ and type I Service level $=96.5 \%$, using the following data: $\mathrm{A}=15, \mathrm{~h}=30, \mathrm{p}=40$, Normal Distribution during $\mathrm{LT}=1.5$ months, with pdf $\mathrm{N}(\mu, \sigma)=\mathrm{N}(1.726,1.314)$. They use a different formula of the one used by Brandimarte and Zotteri, but they, as well, do not consider the "pump" of fig. 13.

Using the same data, and the right ideas (as sketched in fig. 13), F. Galetto finds $\mathrm{Q}=2, \mathrm{R}=4$ and type I Service level $=98.69 \%$ : lower costs for better service level!!!! This result comes from the right formulae.

I got this "advert". It can be related to the following example. 
Factory Physics Forum May 2006

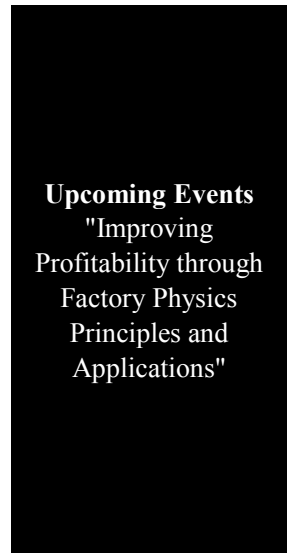

Factory Physics for Managers A series of articles designed to help managers and executives apply practical science to cut through confusion and conflict to get best possible control and optimization of manufacturing and supply chain operations. Understanding Your Value Stream in a Hurry. The full set of Lean Physics Support Tools provides standard applications for easily applying Factory Physics principles in complex manufacturing and supply chain environments.

"I know of no quicker more effective approach to improving performance and profitability." -- a Fortune 500 company manufacturing executive

Notice that the book Factory Physics is used all over the world in many universities. It is cited very many times!!!

How many students, all over the world, are learning wrong methods and will, in theirfuture work, take wrong decisions?

These and the future examples show very clearly Deming statements:

$\Rightarrow$ "The result is that hundreds of people are learning what is wrong. ... I make this statement on the basis of experience, seeing every day the devastating effects of incompetent teaching and faulty applications." "It is a hazard to copy", "It is necessary to understand the theory of what one wishes to do or to make."

It is important to notice that you do not need the right formula to understand that a wrong formula is wrong. Any formula that does not consider the "pump" (in fig. 13) is wrong! How many professors do not use "pump" (in fig. 13) for their optimisation?

The statement of the Nobel prize M. Gell-Mann is relevant: "Once that such a misunderstanding has taken place in the publication, it tends to become perpetual, because the various authors simply copy one each other."... >>\{ "The Quark and the Jaguar: Adventures in the Simple and the Complex" [W. Freeman and Company, N. Y., 1994]\}

In order to let the reader understand all the problems with inventory management, as provided to students in universities, let's see the 6 steps that are used in distribution logistic courses.

$1^{\text {st }}$ step: the case of "constant (fixed)" demand The oldest, and simplest, model is the "the Economic Order Quantity", named EOQ model; it's the way to the more sophisticated ReOrder Level (ROL) model. One of the earliest applications of mathematics to factory management was the work of F. W. Harris (1913) on the problem of setting manufacturing lot sizes. He made the following assumptions about the manufacturing system: 1) production is instantaneous, 2) delivery is immediate, 3) a production run incurs a fixed setup cost, 4) there is no interaction between different products, 5) demand is deterministic, 6) demand is constant over time.

NOTICE: due to limited space allowed for the paper we use the following figures 14 and 15 for all the six cases. The reader must mentally adapt them to the various cases [F. Galetto begs pardon]

Let's consider the problem of establishing the order quantity Q [lot size] for an inventory system, dealt in "Logistics courses" and related books. In this field the assumptions are very similar: a single item is subject to "constant (fixed)" demand " $\lambda$ " [demand rate, in units per year; in this case the distance between peaks is constant], there is a fixed cost A [ordering cost, in euro] of placing an order and a carrying charge " $\mathrm{h}$ " [holding cost, in euro per unit, per unit time allotted (often year) to each item in inventory]. If no stockouts are permitted and lead time is zero (i.e. orders arrive immediately) there is a quantity Q (named EOQ: Economic Order Quantity), given by the famous Wilson lot-size formula $Q=\sqrt{2} A \lambda / h$ that minimise the "total cost per unit time (often per year)"; (NOTICE that actually the books name it as total cost [forgetting "per unit time”]). The inventory can be depicted as a system that starts with $\mathrm{Q}$ units (the level, I, of the inventory, being $\mathrm{ROL}=0$ ): we are certain that $\lambda t$ units are sold (delivered) in any interval of duration $\mathrm{t}$; when the level inventory is zero, $\mathrm{I}=0, \mathrm{Q}$ products are ordered and arrive immediately $(\mathrm{LT}=0) \ldots$ and the system starts again from scratch.

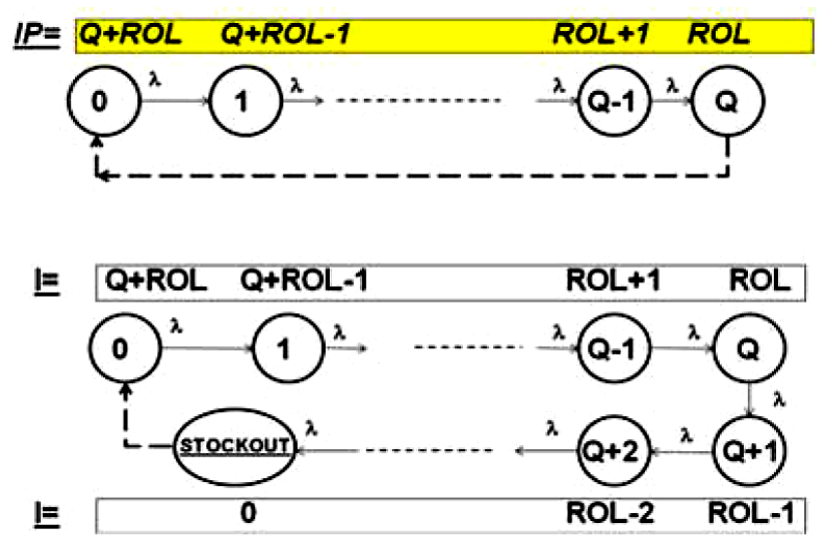

Figure 14. System inventory states; state i means i products dispatched. ROL is the ReOrder Level, $\lambda$ is the selling rate (it can also depends on time $t$; IP is the Inventory Position, while I is the actual Inventory

The function depicting the curve of the Inventory Position $\mathrm{IP}(\mathrm{t})$ is a saw-tooth line, with random distance between peaks.

$\mathbf{I P}(\mathbf{t})$

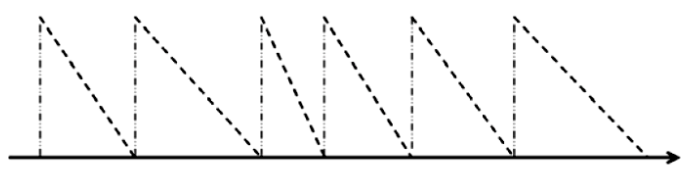

Figure 15. Level of Inventory Position versus time t, random distance between peaks.

The production cost does not influence the solution and therefore in not considered in the "total cost per year" $\mathrm{Y}(\mathrm{Q})=$ $\mathrm{hQ} / 2+\mathrm{A} \lambda / \mathrm{Q}$. Taking the derivative of $\mathrm{Y}(\mathrm{Q})$, and using elementary concepts of calculus, one gets easily the Wilson formula $Q=\sqrt{2 A \lambda / h}$. In this particular case, I repeat, in 
this particular case, the number of lots ordered per year is $\mathrm{N}=\lambda / \mathrm{Q}$ and the optimal time between orders is $\mathrm{T}=\mathrm{Q} / \lambda$, i.e. $\mathrm{T}=1 / \mathrm{N}$.

Notice what happened in a MASTER (after 5 years of Engineering courses) on Maintenance and Reliability, in the lessons for RCM [Reliability Centred Maintenance]: Wilson formula $Q=\sqrt{2 A \lambda / h}$, which holds only in the hypotheses we said just before, was provided to student for buying the spare parts, which obviously depend on the number of failures, which obviously depend on the unreliability, which obviously depend on the time failure, which obviously is a random variable!!! A serious teacher should have proved that the formula holds true, before teaching it to students !!!!

$2^{\text {nd }}$ step: the case of random demand with "constant" demand rate and steady state of the stochastic process We are going now to consider the demand as a random variable, so introducing the need of the use of probability theory. If we maintain all the previous hypotheses, but the number 5 and 6 : 1) production is instantaneous, 2) delivery is immediate, 3 ) a production run incurs a fixed setup cost, 4) there is no interaction between different products, 5) demand is random, 6) demand rate is constant over time. ( fig 14)

We can depict the system as before where now the "time to sell a new unit (time between demands)" is a random variable exponentially distributed.

The function depicting the curve of the inventory level I $(\mathrm{t})$ is a saw-tooth line, with variable [exponential random] time distance between peaks. Therefore the probabilistic structure of the inventory system is a Markov process, periodic with period Q. The mean time (holding time) in any state is $m=1 / \lambda$, the steady-state transition probability from one state $i$, to the next state $\mathrm{i}-1$ is constant $\varphi_{\mathrm{i}}=\varphi_{\mathrm{i}-1}=1 / \mathrm{Q}$ [use Markov chains theory]. The "reward structure" is such that the order cost A is associated with the transition from state $\mathrm{Q}$ to state 0 , while the holding cost, per unit time, for state $\mathrm{i}$ is $\mathrm{y}_{\mathrm{i}}=(\mathrm{Q}-\mathrm{i})^{*} \mathrm{~h}, \mathrm{i}=2$ to $\mathrm{Q}$, and $\mathrm{y}_{1}=\mathrm{h}+\lambda \mathrm{A}$; the average cost per unit time, $\mathrm{g}(\cos \mathrm{t}$ rate) for operating the system in the steady state is $g=[\lambda A+h Q(Q+1) / 2] / Q$

The value $\mathrm{Q}$ that optimises the cost rate, in the steady state of the stochastic process, i.e. when the time is tending to infinity, is found as the solution of the previous equation. If $\mathrm{Q}$ is large, one can ignore the discrete nature of Q [Q is an integral number], assuming it can be considered as a continuous variable: so we can differentiate and set the derivative equal to zero; the solution is (the famous Wilson lot-size formula) $Q=\sqrt{2 A \lambda / h}$. If $\mathrm{Q}$ is small, we cannot ignore the discrete nature of $\mathrm{Q}$ [Q is an integral number], and the solution has to be find numerically. [remember the case of citations....]

$3^{\text {rd }}$ : the case of random demand with "variable" demand rate and steady state of the stochastic process We are going to consider again the demand as a random variable, (need of probability theory), maintaining all the previous hypotheses [as in the $2^{\text {nd }}$ case], but the number $6: 1$ ) production is instantaneous, 2) delivery is immediate, 3 ) a production run incurs a fixed setup cost, 4) there is no interaction between different products, 5) demand is random, 6) demand rate is
NOT constant over time, but it varies with time, identically after any transition from a state to the following one.

We can depict, again, the system as before (see fig. 14) where now the "time to sell a new unit (time between demands)" is a random variable "identically" [but not exponentially, as it was before] distributed; let indicate the probability density of the time between transitions as $\mathrm{f}(\mathrm{t})$ [related to the "rate" $\lambda(t)$, with cumulative distribution $F(t)$ ]; its mean is $\mathrm{m}$.

The mean number of state transitions in the interval $0^{------t}$, $M(t)$ is the solution of the integral equation

$$
M(t)=F(t)+\int_{0}^{t} f(r) M(t-r) d r
$$

The related intensity of state transitions, at time $t$, is $\mathrm{m}(\mathrm{t})=\mathrm{dM}(\mathrm{t}) / \mathrm{dt}$, the solution of the integral equation

$$
m(t)=f(t)+\int_{0}^{t} f(r) m(t-r) d r
$$

In the process steady state we have [see Reliability Theory (e.g. F. G. books] $M(t) \cong t / m$ and $m(t)) \cong 1 / m$, for $t \rightarrow \infty$. The function depicting the curve of the inventory level $\mathrm{I}(\mathrm{t})$ is a saw-tooth line, with variable [randomly] time distance between peaks. Therefore the probabilistic structure of the inventory system is a semi-Markov process, periodic with period $\mathrm{Q}$. The mean time (holding time) in any state is $\mathrm{m}$ [the mean of the distribution] identical for all the states; then the steady-state transition probability from one state $i$, to the next state i-1 is constant $\varphi_{\mathrm{i}}=\varphi_{\mathrm{i}-1}=1 / \mathrm{Q}$ [use semi-Markov processes theory]. The "reward structure" is such that the order cost $\mathrm{A}$ is associated with the transition from state $\mathrm{Q}$ to state 0 , while the holding cost, per unit time, for state $i$ is $y_{i}=$ (Q-i)*h, i=2 to $\mathrm{Q}$, and $\mathrm{y}_{1}=\mathrm{h}+\mathrm{A} / \mathrm{m}$; the average cost per unit time, $\mathrm{g}$ (cost rate), for operating the system in the steady state is the same, as before, $g=[A / m+h Q(Q+1) / 2] / Q$.

The value $\mathrm{Q}$ that optimises the cost rate, in the steady state of the stochastic process, i.e. when the time is tending to infinity, is found as the solution of the previous equation. If $\mathrm{Q}$ is large, one can ignore the discrete nature of $\mathrm{Q}[\mathrm{Q}$ is an integral number], assuming it can be considered as a continuous variable: so we can differentiate and set the derivative equal to zero; the solution is $Q=\sqrt{2 \mathrm{~A} /(\mathrm{mh})}$ (similar to the famous Wilson lot-size formula); if different types of distributions are used, but with the same mean, one gets the same optimum g. If $Q$ is small, we cannot ignore the discrete nature of Q [Q is an integral number], and the solution has to be find numerically. [remember the case of citations....]

Notice that we can manipulate the formula, obtaining the following $g=\frac{A}{m Q}+\frac{h m Q(Q+1) / 2}{m Q}$ that shows very clearly a fundamental fact of renewal processes: the gain rate, in the steady state of a process, is the ratio of the cost during a renewal cycle and the length of the cycle $[\mathrm{mQ}$, that is the mean of the sum of $\mathrm{Q}$ random variables, identically distributed]; we will find the same idea in the formulae of preventive maintenance. 
Notice that nobody says that the formulas in the various books and papers are to be considered only for the steady state.

It is very interesting noting that, after a long time $t^{*}$, at which the stochastic process reaches "almost surely" its

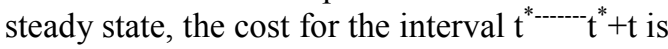

$$
g t=[A t / m+Q(Q+1) h t / 2] / Q
$$

which shows that $\mathrm{t} / \mathrm{m}$ is the mean number of orders for the interval $t^{*---t^{*}+t}$ (in the steady state) and $Q(Q+1) h t / 2$ is the mean number of products, for holding which we pay, for the

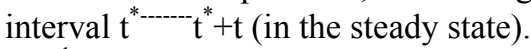

$4^{\text {th }}$ step: the case of random demand with "constant" demand rate and steady state of the stochastic process. We are going now to consider the demand as a random variable, so introducing the need of the use of probability theory, but we consider a lead time different from 0 , we maintain some of the previous hypotheses, but the number 2, 5 and $6: 1$ ) production is instantaneous, 2) delivery takes a constant time $L$, named Lead Time, after the order, 3) a production run incurs a fixed setup cost, 4) there is no interaction between different products, 5) demand is random, 6) demand rate is constant over time. We can no longer depict the system as before; we need to distinguish between the net inventory $\mathrm{I}(\mathrm{t})$ and the inventory position $\operatorname{IP}(\mathrm{t})$. The net inventory $\mathrm{I}(\mathrm{t})$ is the actual number of products we have on hand that we can send to our customers, after a time $\mathrm{L}$, form their order. The inventory position $\operatorname{IP}(t)$ is the sum of $I(t)$, the actual number of products we have on hand, the outstanding orders not yet arrived at time $t$, minus the products backlogged.; the order of $Q$ products is placed, at any time $t_{0}$, when $\operatorname{IP}\left(\mathrm{t}_{0}\right)$ equals the ROL (the Re-Order Level); unfortunately, in the meantime [duration L] a stockout might occur: while we wait for the lot arrival (replenishment of the inventory), at time $t_{0}+\mathrm{L}$, the net inventory $I(t)$ and the inventory position $\operatorname{IP}(t)$ decrease because of selling (and dispatching) products. If it happens that $\mathrm{I}\left(\mathrm{t}_{\mathrm{STO}}\right)=0$, at a time $\mathrm{t}_{\mathrm{STO}}$, we face an inventory STockOut that generates a cost: customers are unsatisfied...; we lose to sell products, a case named "Lost Sales". The cost involved in this case are: the order cost $\mathrm{A}$, the cost of holding the inventory (that varies with time, and the "penalty cost" due to stockout. The "time to sell a new unit (time between demands)" is a random variable exponentially distributed. The function depicting the curve of the Inventory Position level IP(t) is a saw-tooth line, with variable [randomly] time distance between peaks, exponentially distributed.

Also in this case the probabilistic structure of the inventory system is a Markov process, periodic with period Q. The mean time (holding time) in any state is $m=1 / \lambda$, the steady-state transition probability from one state $\mathrm{IP}=\mathrm{i}$, to the next state $\mathrm{IP}=\mathrm{i}-1$, in the process steady state, is still constant $\varphi_{\mathrm{i}}=\varphi_{\mathrm{i}-1}=1 / \mathrm{Q}$ [use Markov chains theory].

The "reward structure" [see fig. 14] is such that the order cost $\mathrm{A}$ is associated with the transition from state $\mathrm{Q}+\mathrm{ROL}$ and ROL; the carrying inventory cost is associated with the mean number of products on hand time the time they are in the inventory, while the stockout cost is related to the probability that happens the event $I(t)=0$, in spite that we have ROL product when we order the lot of Q products. [we will use, for short, $\mathrm{R}$ for the ROL, ReOrder Level]

Let $\mathrm{t}_{0}$ be the time instant when $\operatorname{IP}\left(\mathrm{t}_{0}\right)=\mathrm{R}$; the net inventory $\mathrm{I}\left(\mathrm{t}_{0}+\mathrm{L}\right)=\mathrm{R}$ - demanded quantity $\mathrm{X}_{\mathrm{L}}$, during the lead time $\mathrm{L}$, is a random variable with the same type of distribution as Inventory Position IP $\left(t_{0}+L\right)$; for any interval $t_{0}^{-----1} t_{0}+\Delta t$ the holding cost is a random variable as well

$$
h \int_{t_{0}}^{t_{0}+\Delta t} I(u) d u=h \operatorname{TTI}\left(t_{0}^{----} t_{0}+\Delta t\right)
$$

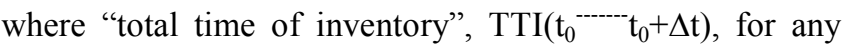
interval $t_{0}-{ }^{--} t_{0}+\Delta t$, the time for which we have to pay for the products we have on hands [net inventory ] and for the time they are on hands, with mean

$$
h \int_{t_{0}}^{t_{0}+\Delta t} E[I(u)] d u=h E\left[T T I\left(t_{0}^{----} t_{0}+\Delta t\right)\right]
$$

Being $I(t)=I P(t)-Q$, for any time $t$, the "total time of inventory", TTI $\left(\mathrm{t}_{0}-\mathrm{t}_{0}+\Delta \mathrm{t}\right)$ depends on the transitions between the states $0,1,2, \ldots, \mathrm{Q}$ and the related probabilities. Therefore the mean of the random variable TTI is

$$
\begin{aligned}
& E\left[T T I\left(t_{0}^{----} t_{0}+\Delta t\right)\right]= \\
& \frac{\Delta t}{\lambda}\{(R+Q)+(R+Q-1)+(R+Q-1)+\ldots .+(R+1)\}-Q \Delta t
\end{aligned}
$$

Using simple concepts of Algebra, we get

$$
\begin{aligned}
& E\left[T T I\left(t_{0}{ }^{----} t_{0}+\Delta t\right)\right]= \\
& \frac{\Delta t}{2 \lambda}\{(R+Q)(R+Q+1)-R(R+1)\}-Q \lambda \Delta t / \lambda
\end{aligned}
$$

Being $T_{Q}$ the random time for selling $Q$ products, and so reorder a new lot of products, we have, for any planning cycle $\mathrm{t}_{0}-\mathrm{t}_{0}+\mathrm{T}_{\mathrm{Q}}$

$$
E\left[T T I\left(t_{0}{ }^{----} t_{0}+T_{Q}\right)\right]=Q\{R+(Q+1) / 2-\lambda L\} / \lambda
$$

Therefore the expected cost of inventory is

$$
h Q\{R+(Q+1) / 2-\lambda L\} / \lambda
$$

We proved this formula using probability theory; in all the books F. Galetto read, never he found the proof! Why?

The quantity $s s=R-\lambda L$ is the safety stock that we hold in order to prevent stockouts. If $t_{0}$ is the time instant when $\operatorname{IP}\left(\mathrm{t}_{0}\right)=\mathrm{R}$; the stockout happens when the net inventory I $\left(\mathrm{t}_{0}+\mathrm{L}\right)$ $=\mathrm{R}$ - demanded quantity $\mathrm{X}_{\mathrm{L}}$, during the lead time $\mathrm{L}$, which is a random variable, falls below zero: $\mathrm{P}_{\mathrm{STO}}=\mathrm{P}\left[\mathrm{I}\left(\mathrm{t}_{0}+\mathrm{L}\right) \leq 0\right]$; it means that, at some instant, $\mathrm{t}_{\mathrm{STO}} \leq \mathrm{t}_{0}+\mathrm{L}, \mathrm{I}\left(\mathrm{t}_{0}+\mathrm{t}_{\mathrm{STO}}\right)=0$. Letting $T_{R}$ be the random time for selling $\mathrm{R}$ product, we have $\mathrm{P}_{\mathrm{STO}}=\mathrm{P}\left[\mathrm{T}_{\mathrm{R}} \leq \mathrm{L}\right]$.If $\mathrm{T}_{\mathrm{R}}>\mathrm{L}$ the system is able to provide products, we have on hand (net inventory), to all customers asking for them, filling their demands; that's why the probability $\mathrm{S}(\mathrm{R}, \mathrm{L})=\mathrm{P}\left[\mathrm{T}_{\mathrm{R}}>\mathrm{L}\right]=1-\mathrm{P}_{\mathrm{STO}}=$ is named Service Level (type 1), or Fill Rate. Noting that $\mathrm{P}\left[\mathrm{T}_{\mathrm{R}}>\mathrm{L}\right]$ is the "reliability of a stand-by system of R products" failing with failure rate equal to $\lambda$, one can take advantage of the use of all the ideas of Reliability Theory for the field of Inventory 


\section{Management.}

Here we are doing that.

Let $\mathrm{T}_{\text {STO }}$ be the random variable "Time To Stock Out" of the inventory system and $\mathrm{N}_{\mathrm{STO}}(\mathrm{t})$ be the random variable "Number of Stock Outs" of the system, in the interval $0^{------t}$; at time $\mathrm{t}$ the system has a "residual life" $\rho(\mathrm{t})$ until the next Stock Out, $\rho(t)=T_{N_{\text {STO }}(t)}-t$; since the transitions depend on the exponential distribution $\rho(t)$ is independent from the Number of the experienced Stockouts. Let $S(R, t+x \mid t)=$ $P[\rho(t)>x]$ be the type 1 Service Level, related to the interval $\mathrm{t}^{-----} \mathrm{t}+\mathrm{x}$; F. Galetto proved (chapter 6 of [13]Affidabilità...) that the type 1 Service Level $S(R, t+x \mid t)=P[\rho(t)>x]$ is the solution of the integral equation

$$
S(R, t+x \mid t)=S(R, t+x \mid 0)+\int_{0}^{t} f_{S T O}(s) S(R, t+x \mid s) d s
$$

where $f_{\text {STO }}(t)$ is the probability density function of the $1^{\text {st }}$ $\mathrm{T}_{\mathrm{STO}}$, with mean denoted as MTTSTO and named Mean Time To STockOut.

If $\mathrm{t} \rightarrow \infty$ the type 1 Service Level $\mathrm{S}(\mathrm{R}, \mathrm{t}+\mathrm{x} \mid \mathrm{t})$ depends only on $\mathrm{x} ; \mathrm{F}$. Galetto proved (chapter 6 of [13]Affidabilità...) that $\mathrm{S}(\mathrm{R}, \mathrm{x})=\mathrm{P}[\rho(\infty)>\mathrm{x}]$ is related to the density of stock outs $\mathrm{f}_{\mathrm{STO}}(\mathrm{x} \mid \infty)=\mathrm{S}(\mathrm{R}, \mathrm{x} \mid 0) /$ MTTSTO.

Therefore, after a long time that the inventory system is running, the steady state type 1 Service Level $S(R, L)$ is

$$
S(R, L)=\int_{L}^{\infty} S(R, s) d s
$$

What one can find in documents? The two following excerpts (pages 277 and 307) are copied directly from books; it is not important to report the names of the authors! None of the authors say that their formulae hold only in the steady state of the process. Notice that a lot of attention is needed in order to find the correspondence between the different notations.

FIXED QUANTITY: THE $(Q, R)$ MODEL
Under these assumptions the total cost is
\[ \begin{aligned} C_{\text {tot }}= & C_{o r}+C_{i n}+C_{s o} \\ = & A \cdot \frac{\mathrm{E}(d)}{Q}+h \cdot(R+Q / 2-\mathrm{E}(d) \cdot L T) \\ & +p \cdot \frac{\mathrm{E}(d)}{Q} \cdot\left(1-F_{d_{L T}}(R)\right) .\end{aligned} \]

From a book one can find, where $\mathrm{d}$ is the random demand, LT is the lead time, $\mathrm{F}_{\mathrm{dLT}}(\mathrm{R})$ is the cumulative probability of sales during LT, $\mathrm{p}$ is the cost (penalty) of stock out. Notice that there is no proof of this formula in the book. From another book one can find, where CSL is the Cycle Service Level [i.e. the fraction of replenishment cycles that end with all customer demand being met (a replenishment cycle is the interval between two successive replenishment deliveries). The CSL is equal to the probability of not having a stockout in a replenishment cycle.], $\mathrm{H}$ is the cost of holding one item for one unit of time, $C_{u}$ is the cost of one item, D is the average demand for one unit of time.
In the notations of the previous book $D_{L}=E(d), H=h$, $\mathrm{ROP}=\mathrm{R}$, replenishment cycle is equal $\mathrm{LT}, \mathrm{CSL}$ is then $\mathrm{F}_{\mathrm{dLT}}(\mathrm{R}), \mathrm{ss}=\mathrm{R}-\lambda \mathrm{L}$ (safety stock).

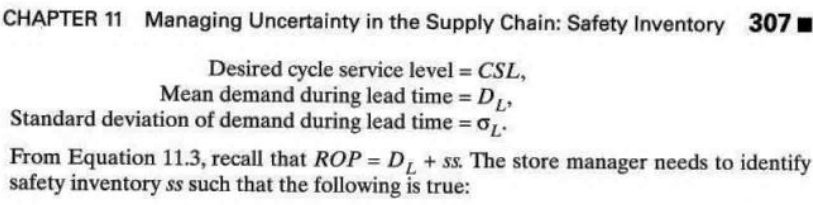

From Equation 11.3, recall that $R O P=D_{L}+s s$. The store manager needs to identify safety inventory ss such that the following is true:

Probability (demand during lead time $\left.\leq D_{L}+s s\right)=C S L$.

Demand During Stockout Is Lost

For the case where unfilled demand during the stockout period is lost, the optimal cycle service level $C S L^{*}$ is given as follows:

$$
C S L^{*}=1-\frac{H Q}{H Q+D C_{u}}
$$

In this case we have assumed that $C_{u}$ is the cost of losing one unit of demand during the stockout period. In Example 12.4, we evaluate the optimal cycle service level if demand is lost during the stockout period.

From another book, again, one can find, where $\mathrm{G}(\mathrm{r})=\mathrm{S}(\mathrm{Q}$, $r$ ) is the Service Level (type 1$), I(Q, r)$ is the average net inventory, D is the expected demand per year (in units), $\mathrm{k}$ is the cost per stockout.

In the notations of the previous book $\mathrm{D}=\mathrm{D}_{\mathrm{L}}=\mathrm{E}(\mathrm{d}), \mathrm{h}=\mathrm{H}=\mathrm{h}$, $\mathrm{r}=\mathrm{ROP}=\mathrm{R}, \mathrm{S}(\mathrm{Q}, \mathrm{r})=\mathrm{CSL}=\mathrm{F}_{\mathrm{dLT}}(\mathrm{R}), \mathrm{k}=\mathrm{p}$. Notice that that the three books provide to the students (or to the managers) three different formulae for the same concept, the type 1 Service Level!!!

It is very clear that it is very improbable that the cost per stockout is equal to the cost per unit.

Stockout Cost Approach. As an alternative to the backorder cost approach, we can make verbal formulation (2.32) into a mathematical model by writing the sum of the annual setup or purchase order cost, stockout cost, and inventory carrying cost as

$$
Y(Q, r)=\frac{D}{Q} A+k D[1-S(Q, r)]+h I(Q, r)
$$

Going through the usual optimization procedure (taking the derivative with respect to $r$, setting the result equal to zero, and solving for $r$ ) yields the following expression for the optimal reorder point:

$$
G\left(r^{*}\right)=\frac{k D}{k D+h Q}
$$

A case from Factory Physics, a book very much used in universities

"Jack, the maintenance manager, has collected historical data that indicate one of the replacement parts he stocks has annual demand (D) of 14 units per year. The unit cost $c$ of the part is $\$ 150$, and since the firm uses an interest rate of 20 percent, the annual holding cost $h$ has been set at $0,2^{*}(\$ 150)=\$ 30$ per year. It takes 45 days to receive a replenishment order, so average demand during a replenishment lead time is $\theta=\frac{14}{365} \cdot 45=1,726$ " [units/lead time] "The part is purchased from an outside supplier, and Jack estimates that the cost of time and materials required to place a purchase order $A$ is about $\$ 15$. The one remaining cost required by our model is the cost of either a backorder or stockout. Although he is very uncomfortable trying to estimate these, when pressed, Jack made a guess that the annualized cost of a backorder is about $\$ 100$ per year, and the cost per stockout event can be approximated by $k=\$ 40$. Finally, Jack has chosen to model demands using the Poisson distribution.

[...] The order quantity is computed by using " [the Economic Order Quantity formula], "which yields $Q^{*}=\sqrt{\frac{2 A D}{h}}=\sqrt{\frac{2 \cdot(15) \cdot(14)}{30}}=3,7 \approx 4$ units." 
Table 4. various formulae for Service level in Inventory Management

\begin{tabular}{|l|l|l|l|}
\hline Book & $\begin{array}{l}\text { Formula for Service } \\
\text { Level }\end{array}$ & $\begin{array}{l}\text { Equivalence } \\
\text { only IF }\end{array}$ & $\begin{array}{l}\text { Equivalence } \\
\text { only IF }\end{array}$ \\
\hline 1 & $\mathrm{~F}_{\mathrm{dLT}(\mathrm{R})}$ & $\begin{array}{l}\mathrm{F}_{\mathrm{dLT}}(\mathrm{R})=1- \\
\mathrm{HQ} /(\mathrm{HQ} \\
\left.+\mathrm{DC}_{\mathrm{u}}\right)\end{array}$ & $\begin{array}{l}\mathrm{F}_{\mathrm{dLT}}(\mathrm{R})= \\
\mathrm{kD} /(\mathrm{kD}+\mathrm{hQ})\end{array}$ \\
\hline 2 & $\mathrm{CSL}=1-\mathrm{HQ} /\left(\mathrm{HQ}+\mathrm{DC}_{\mathrm{u}}\right)$ & $\mathrm{DC}_{\mathrm{u}}=\mathrm{kD}$ & $\left.\begin{array}{l}1-\mathrm{HQ} /(\mathrm{HQ} \\
+\mathrm{DC}\end{array}\right)=\mathrm{F}_{\mathrm{dLT}}(\mathrm{R})$ \\
\hline 3 & $\mathrm{G}(\mathrm{r})=\mathrm{kD} /(\mathrm{kD}+\mathrm{hQ})$ & $\begin{array}{l}\mathrm{kD} /(\mathrm{kD}+\mathrm{hQ})= \\
\mathrm{F}_{\mathrm{dLT}}(\mathrm{R})\end{array}$ & $\mathrm{kD}=\mathrm{DC}_{\mathrm{u}}$ \\
\hline
\end{tabular}

Let's provide clearly the relevant data: annual demand $\mathrm{D}=14$. NOTICE "estimated from historical data", without any confidence interval!, Lead time $\mathrm{L}=45$ days cost of order $A=15 \$$, holding cost $h=30 \$$ per unit per year stockout cost $k=40$ \$, demand distribution: Poisson. Since the demand distribution is Poisson, the time between demands is exponentially distributed, and the system can be modelled with a Markov chain in the steady state of the process. On the contrary, the Factory Physics authors "approximate the Poisson by the normal, with mean 1.726 and standard deviation $\sigma=1.314$ "; then they compute $\mathrm{Q}=3.7(\cong 4)$ and $\mathrm{r}=2.946(\cong 3)$ [with the formula $\mathrm{G}(\mathrm{r})=\mathrm{kD} /(\mathrm{kD}+\mathrm{hQ})]$.

Using "reliability theory", we draw the transition diagram, with transition (selling) rate $\lambda$ [solid lines] and replenishment [dotted lines]; in the steady state we can write the steady transition probability matrix $\mathrm{P}$ that provides us with the MTTSTO, the Cost per Unit Time, the Service Level. We compared our findings with the ones of the Factory Physics authors who "approximate the Poisson by the normal,...": while the Factory Physics authors found a type 1 Service Level $=0.824$, we found 0.903 a better value. We considered also other couples of values for $\mathrm{Q}$ and $\mathrm{R}$ and we found again better results; we provide the readers all the transition diagrams (fig. 16, 17, 18, 19).We use then the exponential distribution because we accepted that the "arrival of failures" was according a Poisson distribution: this implies that the reliability of each item is exponential with failure rate $\lambda / \mathrm{N}$, where $\mathrm{N}$ is the number of items in use; the "Mean Number of Failures in the interval $0^{----t "}, M(t)$, is equal to $\lambda t$ and the variance is $\lambda \mathrm{t}$, as well. The rate $\lambda$ is shown in the transition state diagram; the dotted lines show the replenishments, in the various cases.

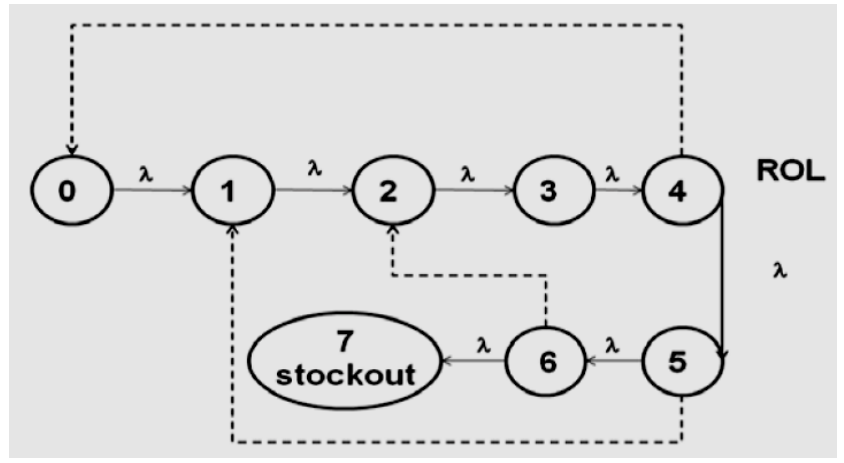

Figure 16. System inventory states (random demand, constant rate); $\mathrm{Q}=4$, $\mathrm{R}=3$

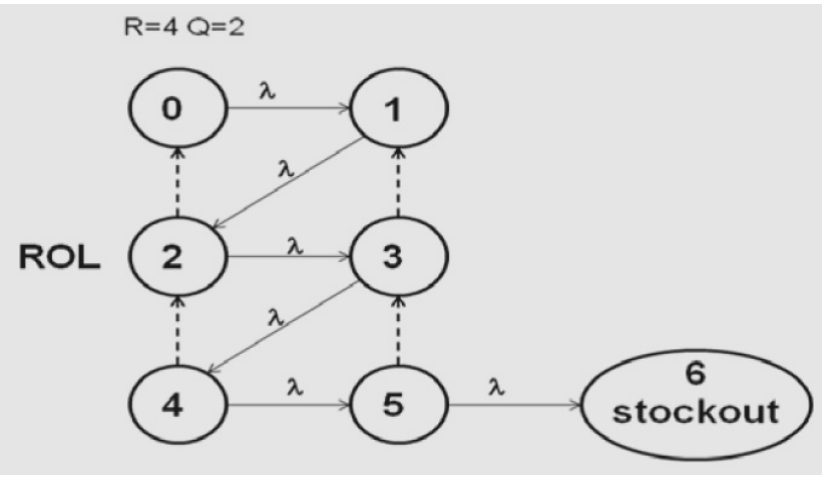

Figure 17. System inventory states (random demand, constant rate); $\mathrm{Q}=2$, $\mathrm{R}=4$

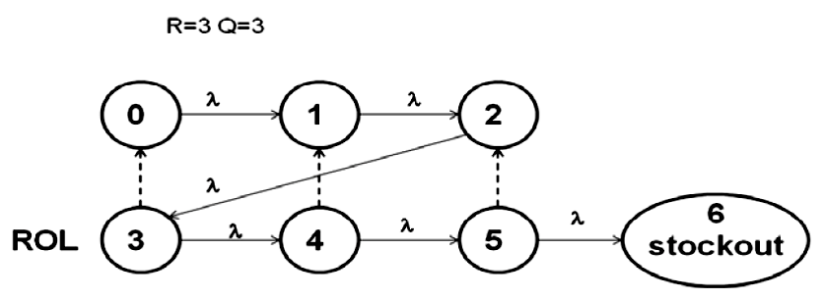

Figure 18. System inventory states (random demand, constant rate); $Q=3$, $\mathrm{R}=3$

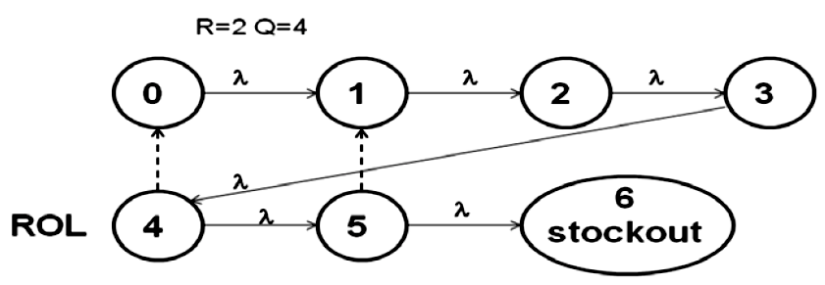

Figure 19. System inventory states (random demand, constant rate); $\mathrm{Q}=4$, $\mathrm{R}=2$

The results, in the steady state, are

Table 5. various results for Cost Rate, Service level in Inventory Management

\begin{tabular}{|c|c|c|c|c|}
\hline $\mathrm{R}=$ & 4 & 3 & 2 & 3 \\
\hline $\mathrm{Q}=$ & 2 & 3 & 4 & 4 \\
\hline Cost rate (\$/year) & $\mathbf{2 3 0 . 5 8}$ & $\mathbf{2 0 3 . 2 4}$ & $\mathbf{1 7 8 . 3 0}$ & $\mathbf{1 6 7 . 3 2}$ \\
\hline Service Level & $\mathbf{0 . 9 6 9}$ & $\mathbf{0 . 9 0 3}$ & $\mathbf{0 . 7 5 0}$ & $\mathbf{0 . 9 0 3}$ \\
\hline
\end{tabular}

It is easily seen that $\mathrm{Q}=2$ and $\mathrm{R}=4$ provide better Service Level ( $97 \%$ vs $82 \%$ found by FP) at a higher cost per year, in the steady state. In case of failures in a production line the cost of unavailability is much higher than $40 \$$... ! It is interesting to notice that the Factory Physics authors did not find that $\mathrm{Q}=2$ and $\mathrm{R}=4$ is the best solution, provided that $97 \%$ of Service Level is considered adequate. In any case it is really better than the solution given to students by the Factory Physics authors.

The distribution of the time to failure of the items was assumed exponential; many times it is not so.

Therefore we are going to develop a method adequate for 
any distribution. In order to do that we will use the following distribution of the "time to sell one item"; we do so because it is related to the normal distribution of the items sold during the time; therefore the inventory $\mathrm{I}(\mathrm{t})$ is a normal stochastic process, during the lead time L: this is the standard assumption in all the books F. Galetto read. The probability density of the time to sell one product is $\left(\mu_{1}\right.$ is the mean time to sell one product)

$$
f\left(t \mid 1, \mu_{1}\right)=e^{-\frac{\left(t-\mu_{1}\right)^{2}}{2 t \mu_{1}{ }^{2}}} / \sqrt{2 \pi t^{3}}
$$

If we consider the time to sell $\mathrm{Q}$ products, $\mathrm{T}_{\mathrm{Q}}$, this has a distribution of the same type, as a result of the convolution of Q $\mathrm{f}(\mathrm{t} \mid 1, \mu)$; we name it as $\mathrm{f}_{\mathrm{Q}}(\mathrm{t} \mid \eta, \mu)$ (where $\mathrm{E}\left[\mathrm{T}_{\mathrm{Q}}\right]=\mu$ and $\operatorname{Var}\left[T_{Q}\right]=\mu^{3} / \eta$. Besides $\eta=Q^{2}$.)

$$
f_{Q}(t \mid \eta, \mu)=\sqrt{\frac{\eta}{2 \pi t^{3}}} e^{-\eta \frac{(t-\mu)^{2}}{2 t \mu^{2}}}
$$

The mean holding time in a state $i$ is $m_{i}$, and steady state transition probabilities, from state $i$ to $j, p_{i j}$ are found in the same manner as in the case of the Poisson process [not with the same formulae, obviously!!!!].

Using this distribution for the previous cases, as obvious, we get quite different steady state results.

The best solution for the steady state cost rate is $Q=4$ and $\mathrm{R}=2$, while for the service level is $\mathrm{Q}=2$ and $\mathrm{R}=4$ (as before).

Table 6. two results for Cost Rate, Service level in Inventory Management

\begin{tabular}{|c|c|c|}
\hline $\mathrm{R}=$ & 4 & 2 \\
\hline $\mathrm{Q}=$ & 2 & 4 \\
\hline Cost rate (\$/year) & 182.27 & 148.10 \\
\hline Service Level & 0.959 & 0.584 \\
\hline
\end{tabular}

$5^{\text {th }}$ step: the case of random demand with "constant" demand rate BUT NOT steady state of the stochastic process

We maintain some of the previous hypotheses, but the number 2, 5 and 6:1) production is instantaneous, 2) delivery takes a constant Lead Time, after the order, 3) a production run incurs a fixed setup cost, 4) there is no interaction between different products, 5) demand is random, 6) demand rate is constant over time. (exponential distribution)

Before we considered the process in its steady state, (after a long time $[\mathrm{t} \rightarrow \infty]$ ); now we consider the case of finite time $\mathrm{t}$.

As in the previous paragraph, we distinguish between the net inventory I $(\mathrm{t})$ and the inventory position IP( $\mathrm{t})$. If, at some instant of time $t_{\text {STO }}, \quad I\left(t_{\text {STO }}\right)=0$, we face an inventory STockOut, that generates a cost: customers are unsatisfied...; we lose to sell products, a case named "Lost Sales": as before, the costs involved are the order cost A, the cost of holding the inventory (that varies with time, and the "penalty cost" due to stockout.

We assume again that the "time to sell a new unit (time between demands)" is a random variable exponentially distributed, with rate $\lambda$; so the Inventory Position level IP(t) is a saw-tooth line, with variable [randomly] time distance between peaks, exponentially distributed [Poisson Process]. Therefore the probabilistic structure of the inventory system is a Markov process, periodic with period Q. Letting $\mathrm{P}_{\mathrm{i}}(\mathrm{t})=\mathrm{P}$ [process in the state $\mathrm{i}$, at time $\mathrm{t}$ ], be the probability that process in the state $i$, at time $t$, we can write a system of differential equation; in order to make the system simple we consider the case $\mathrm{R}=2$ and $\mathrm{Q}=4$. Using the diagram of fig. 19 one finds the equations that rule the stochastic process that can be written in matrix form as

$$
P^{\prime}(t)=A P(t)
$$

where we have the vector $\mathrm{P}(\mathrm{t})$ [its derivative $\mathrm{P}^{\prime}(\mathrm{t})$ ] and the matrix $\mathrm{A}$ of the transition rates, $\lambda$ and $\delta(\mathrm{t}-\mathrm{L})$ ["Dirac impulse"]. Explicitly

$$
\begin{aligned}
& P_{0}^{\prime}(t)=-\lambda P_{0}(t)+\delta(t-L) P_{4}(t) \\
& P_{1}^{\prime}(t)=\lambda P_{0}(t)-\lambda P_{1}(t)+\delta(t-L) P_{5}(t) \\
& P_{2}^{\prime}(t)=\lambda P_{1}(t)-\lambda P_{2}(t) \\
& P_{3}^{\prime}(t)=\lambda P_{2}(t)-\lambda P_{3}(t) \\
& P_{4}^{\prime}(t)=\lambda P_{3}(t)-\lambda P_{4}(t) \\
& P_{5}^{\prime}(t)=-\lambda P_{5}(t)
\end{aligned}
$$

The Service Level $\mathrm{S}(\mathrm{t}, \mathrm{L})$, i.e. no stockout for the interval $0^{----t}$, and Lead Time $\mathrm{L}$ (for replenishment) is $\mathrm{S}(\mathrm{t}, \mathrm{L})=\mathrm{P}(\mathrm{t}) \mathrm{u}$, dot product of the vector $\mathrm{P}(\mathrm{t})$ and the vector $\mathrm{u}=[1,1,1,1,1,1]$.

The instantaneous Inventory Level I(s, L), for any time $\mathrm{s}$ of the interval $0^{----t}$, and Lead Time L (for replenishment) is

$$
I(s, L)=\sum_{0}^{5}(R+Q-i) P_{i}(s)
$$

$I(t, L)=h \int_{0}^{t} I(s, L) d s$ is the cost of Inventory, for the interval $0^{---1} \mathrm{t}$. The Number of Orders is the number of times [random variable] the system enters the state 4 ; its expected value $\mathrm{M}(\mathrm{t})$ is the mean number of orders. The probability of stockout is the probability of entrance in the state 6.Putting all together we have the relationship that provides the cost $\mathrm{C}(\mathrm{t}, \mathrm{L})$ of the system; the cost rate, usually used in books, is here $\mathrm{C}(\mathrm{t}, \mathrm{L}) / \mathrm{t}$; it is in the following figure 20.As we can see the cost rate increases and, after 6 months decreases. The cost rate is lower in the case $\mathrm{Q}=4$ and $\mathrm{R}=2$ with lower service level, as we found in the steady state analysis; both curves decrease very slowly to their steady state rate.

Table 7. two results for Cost Rate, Service level in Inventory Management

\begin{tabular}{|c|c|c|}
\hline $\mathrm{R}=$ & 4 & 2 \\
\hline $\mathrm{Q}=$ & 2 & 4 \\
\hline Cost rate (\$/year) at 6 months & $\mathbf{6 4 3 . 6 9}$ & $\mathbf{3 7 8 . 1 1}$ \\
\hline Service Level at 6 months & $\mathbf{0 . 9 7 4}$ & $\mathbf{0 . 8 6 1}$ \\
\hline
\end{tabular}

If the service level 0.86 , due to the consequences of downtime, is not adequate, we have to choose ... 
It is therefore very important to consider the interval in which we want to optimise.

This subject has been pointed out many times by $\mathrm{F}$. Galetto, in relation with the Preventive Maintenance. We are going to show it because it has an amazing similarity with inventory management.

When the values $\mathrm{Q}$ and $\mathrm{R}$ increase the dimension of the vectors and matrices $P^{\prime}(t)=A P(t)$ increases accordingly.

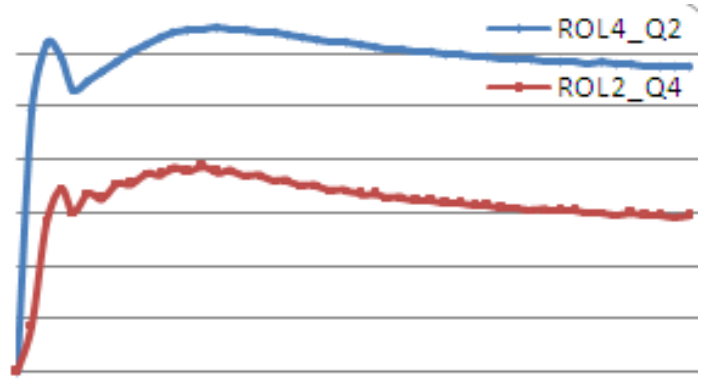

Figure 20. Cost rate of two inventory systems (random demand at constant rate): $\mathrm{Q}=4, \mathrm{R}=2$ vs $\mathrm{Q}=2, \mathrm{R}=4$ [Factory Physics]

Preventive maintenance and RCM, an analogy with inventory management we said before that it is very important the interval in which we want to optimise and that this subject has been pointed out many times by F. Galetto, related to the Preventive Maintenance. We are now going to show it because it has an amazing similarity with inventory management, and shows that there is no need of Poisson processes.

In 1977, at the Reliability \& Maintainability Symposium, Philadelphia, F. Galetto, with his paper "SARA (System Availability and Reliability Analysis)", provided the Reliability Integral Theory that solves various reliability problems that cannot be dealt through Markov processes. The theory did exist, not the single formulae: any scholar could have found them. The same theory Reliability Integral Theory, is applicable to maintenance problems, as those presented in "Some graphical methods for maintenance planning"; in that paper, mentioned before, following ideas of Barlow, the optimum replacement interval is found, by B. Bergman, minimising the formula, cost ratio (given in Barlow; $c_{1}$ is the cost of preventive replacement, $c_{2}$ is the cost due to failures, while $\mathrm{c}$ [Bergman] is the cost of preventive replacement, assuming 1 the cost due to failures: $\mathrm{c}=\mathrm{c}_{1} / \mathrm{c}_{2}$ )

$$
C\left(t_{p}\right)=\frac{c F\left(t_{p}\right)+R\left(t_{p}\right)}{\int_{0}^{t_{p}} R(x) d x},
$$

very similar to

$$
C\left(t_{p}\right)=\frac{c_{1} F\left(t_{p}\right)+c_{2} R\left(t_{p}\right)}{\int_{0}^{t_{p}} R(x) d x},
$$

Barlow and Bergman say that those formulas are valid "in the long run": that means after an infinite time, which is after an infinite number of failures! But for a finite time span $0^{---} \mathrm{t}$ ? The cost ratio is computed as the ratio of the mean cost for one cycle divided by the mean duration of one cycle, as it can be done for renewal processes, in the steady state!

The same is found in logistics books, ... but they do not say ... that it is valid ONLY in the steady state!!!! [see the cases]

You understand all that through the F. Galetto papers (in 1977), SARA at the Reliability \& Maintainability Symposium, Philadelphia, and CLAUDIA, $21^{\text {st }}$ EOQC Conference, Varna (Bulgaria).

Some hints are given here, for preventive maintenance: let $\mathrm{N}_{0}\left(t, t_{\mathrm{p}}\right)$ be the number of replacements of unfailed items and $N_{1}\left(t, t_{p}\right)$ be the number of failed items over the interval $0^{---} t$. They are random variables; their expectations are $\mathrm{M}_{0}\left(\mathrm{t}, \mathrm{t}_{\mathrm{p}}\right)$ and $\mathrm{M}_{1}\left(\mathrm{t}, \mathrm{t}_{\mathrm{p}}\right)$. Let $\mathrm{C}\left(\mathrm{t}, \mathrm{t}_{\mathrm{p}}\right)$ be the total expected cost over the interval $0^{--\mathrm{t}}$, when the unfailed items are "renewed" [at cost $\mathrm{c}_{0}$ ] at their life $\mathrm{t}_{\mathrm{p}}$, and failed items are replaced [at cost $\mathrm{c}_{1} ; \mathrm{c}_{1}$ includes the cost of the consequences of the failure] with new items. It is

$$
\mathrm{C}\left(\mathrm{t}, \mathrm{t}_{\mathrm{p}}\right)=\mathrm{c}_{0} \mathrm{M}_{0}\left(\mathrm{t}, \mathrm{t}_{\mathrm{p}}\right)+\mathrm{c}_{1} \mathrm{M}_{1}\left(\mathrm{t}, \mathrm{t}_{\mathrm{p}}\right)
$$

Let $R\left(t, t_{p}\right)$ be the reliability for the interval $0^{---} t$, when unfailed items are "renewed" at their life $t_{p}$, and $b\left(t, t_{p}\right)$ the related probability density function. To optimise the cost over the interval $0^{---} \mathrm{t}$, we need either to compute $\mathrm{M}_{0}\left(\mathrm{t}, \mathrm{t}_{\mathrm{p}}\right)$ and $\mathrm{M}_{1}\left(\mathrm{t}, \mathrm{t}_{\mathrm{p}}\right)$ or to compute $\mathrm{C}\left(\mathrm{t}, \mathrm{t}_{\mathrm{p}}\right)$ from the following integral equation

$$
C\left(t, t_{p}\right)=c_{0} \sum_{i=1}^{n} R^{i}\left(t_{p}\right)+\int_{0}^{t}\left[c_{1}+C\left(t-s, t_{p}\right)\right] b\left(s, t_{p}\right) d s
$$

where $n$ is such that $n t_{p} \leq t \leq(n+1) t_{p}$ and $R\left(t_{p}\right)$ is the reliability of the item for the period of duration $t_{p}$. [formula derived using the "Integral Theory of Reliability" devised by F. Galetto in 1971]

In the formula the $1^{\text {st }}$ term gives account of unfailed items during the interval $0^{---} \mathrm{t}$ : it excludes that any failure happens. The failures are considered in the integral from 0 to $t$; let the $1^{\text {st }}$ failure occur in an interval $\mathrm{s}^{---} \mathrm{s}+\mathrm{ds}$, with probability $\mathrm{b}\left(\mathrm{s}, \mathrm{t}_{\mathrm{p}}\right) \mathrm{ds}$, and cost $\mathrm{c}_{1}$; the other failures occur in the remaining interval s-- $t$, and their cost is $\mathrm{C}\left(\mathrm{t}-\mathrm{s}, \mathrm{t}_{\mathrm{p}}\right)$ : being $\mathrm{s}$ any instant we integrate over the interval $0^{---} \mathrm{t}$.

It is clear that the optimum interval for the preventive replacement depends on $t$, the duration of the interval $0^{---} t$, over which we want to optimise, and the "cost ratio" $\mathrm{c}_{0} / \mathrm{c}_{1}$. The optimum value $t^{*}$, as found in the literature on preventive maintenance, is the solution of

$$
\mathrm{h}\left(\mathrm{t}_{\mathrm{p}}^{*}\right) \operatorname{MTTF}\left(\mathrm{t}_{\mathrm{p}}^{*}\right)=\mathrm{c}_{1} /\left(\mathrm{c}_{1}-\mathrm{c}_{0}\right)+\left(\mathrm{c}_{0} /\left(\mathrm{c}_{1}-\mathrm{c}_{0}\right)\right) \mathrm{R}\left(\mathrm{t}_{\mathrm{p}}{ }_{\mathrm{p}}\right) / \mathrm{F}\left(\mathrm{t}_{\mathrm{p}}^{*}\right)
$$

which optimise the ratio $\mathrm{C}\left(\mathrm{t}, \mathrm{t}_{\mathrm{p}}\right) / \mathrm{t}$, that, for $\mathrm{t} \rightarrow \infty$, does not depend on $t$. This is not satisfactory, because it does not consider properly probability theory, and it is not said over which period $0^{---} \mathrm{t}$ we want to optimise.

The foundations for finding the solution of the integral equation for a finite time span $\mathrm{t}$ are found using "Integral Theory of Reliability". Let's suppose we have the data on a sample of $n=7$ items; and the time to failure (in the sample) are $60,105,180,300,400,605,890$. The items have IFR (as one can easily find). To find the optimum replacement interval, both Barlow and Bergman use a graphical 
procedure on the TTT-plot: they find the "tangent" to the empirical TTT-plot and passing through the point $(-\mathrm{c}, 0)$ for Bergman while for Barlow is $\left(-c_{2} /\left(c_{1}-c_{2}\right), 0\right)$. The procedure is the same, but the points are different! Why? Assuming a cost $\mathrm{c}=0.9$ for preventive replacement and 1 for failure, Bergman finds $t^{*}{ }_{p}=27.9$

On the contrary, optimising the integral equation (with $\eta$ and $\beta$ estimated from the data), F. Galetto finds, over an interval $0-200$, as optimum interval $t^{*}{ }_{p}=42$. The graph in fig. 23 shows easily that $t^{*}{ }_{p}=42$ is better than $t^{*}{ }_{p}=28$ (approximating 27.9); the optimum interval depends on the interval $0^{--} \mathrm{t}$ : rarely $\mathrm{t}=\infty$ is a good choice!

So we see that for more than 40 years we had the theory to solve various reliability problems (and inventory cases, as well).The same ideas can be used to solve a problem given in example 14.1, page345, of the book Practical Reliability Engineering, Wiley \& Sons (1997). It is written there:

"A flexible cable on a robot assembly line has a time-to-failure distribution which is Weibull, with $\gamma=150 \mathrm{~h}$, $\beta=1.7, \eta=300$ h. If a failure occurs whilst in use the cost of stopping the line and replacing the cable is $\$ 5000$. The cost of replacement during scheduled maintenance is $\$ 500$. If the line runs for 5000 hours a year and scheduled maintenance takes place every week (100 hours), what would be the annual expected cost replacement at one-weekly or two-weekly intervals?"

The solution provided in the book, for $5000 \mathrm{~h}$, is

Table 8. results for Cost Rate in Inventory Management

\begin{tabular}{|c|c|c|c|}
\hline interval $(\mathrm{h})$ & 100 & 200 & 400 \\
\hline $\operatorname{cost} / 5000$ & 5.00 & 3.66 & 7.75 \\
\hline
\end{tabular}

Therefore $200 \mathrm{~h}$ interval is considered the best. Actually it is better to replace every $150 \mathrm{~h}$ : no failures and only preventive replacements.

We made a digression to preventive maintenance problems because their analogy with inventory management; the table shows it

Table 9. relationship between Preventive Maintenance and Inventory Management

\begin{tabular}{|c|c|c|c|}
\hline $\mathrm{M}_{0}\left(\mathrm{t}, \mathrm{t}_{\mathrm{p}}\right)$ & $\begin{array}{c}\text { Mean number of } \\
\text { preventive replacement }\end{array}$ & & Mean number of orders \\
\hline $\mathrm{M}_{1}\left(\mathrm{t}, \mathrm{t}_{\mathrm{p}}\right)$ & $\begin{array}{c}\text { Mean number of } \\
\text { failures }\end{array}$ & & Mean number of Stokouts \\
\hline $\mathrm{c}_{0}$ & $\begin{array}{c}\text { cost of prev. } \\
\text { replacement }\end{array}$ & $\mathrm{A}$ & cost of orders \\
\hline $\mathrm{c}_{1}$ & cost of one failure & $\mathrm{p}$ & cost of a Stockout \\
\hline
\end{tabular}

It is apparent that Reliability Integral Theory will help us in dealing with inventory management.

$6^{\text {th }}$ step: the case of random demand with "NONconstant" demand rate and NOT steady state of the stochastic process

We assume now the following hypotheses: 1) delivery takes a constant Lead Time L, after the order, 2) any order incurs a fixed order cost, A, 3) there is no interaction between different products, 4) demand is random, 6) demand rate is NOT constant at every instant of time.

In the five steps before we considered the process in its steady state, i.e. after a long time [ $t \rightarrow \infty$ ]; now we consider the case of finite time $t$, as done for preventive maintenance.

Letting again $\mathrm{P}_{\mathrm{i}}(\mathrm{t})=\mathrm{P}$ [process in the state $\mathrm{i}$, at time $\mathrm{t}$ ], be the probability that process in the state $i$, at time $t$, we can depict the system with the following transition diagram of figure 23, for the case $\mathrm{R}=2$ and $\mathrm{Q}=4$.

The equations are now integral equations, as in the Reliability Integral Theory; the integral equations have the same structure, whatever is the analytic form of the density $\mathrm{f}(\mathrm{t} \mid 1, \mu)$, that we write simply $\mathrm{f}(\mathrm{t})$; let $\mathrm{g}(\mathrm{s})$ be the probability density of the time to replenishment (so dealing with random Lead Time). Following the Galetto's Reliability Integral Theory ideas we can write, being $\mathrm{R}_{\mathrm{i}}(\mathrm{t}-\mathrm{s})$ the probability of no stockout [service level] if the system enters state i, at time s, and

$$
\bar{F}(t)=1-F(t) \quad \bar{G}(t)=1-G(t) \quad \bar{W}(t)=\bar{F}(t) \bar{G}(t)
$$

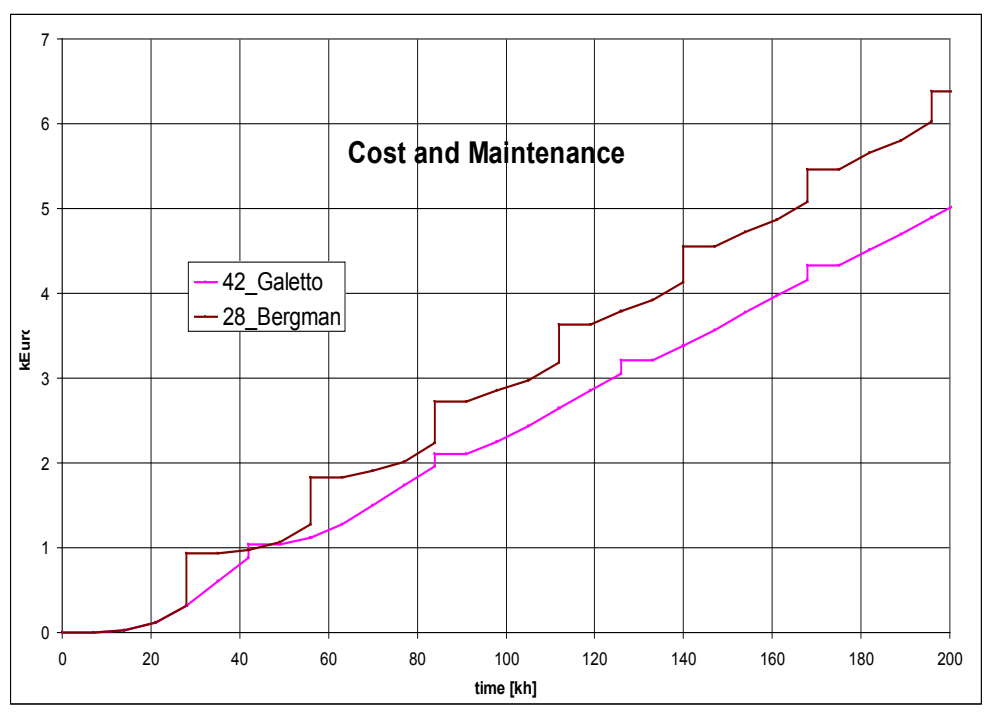

Figure 21. Comparison of "optimum" replacement intervals 

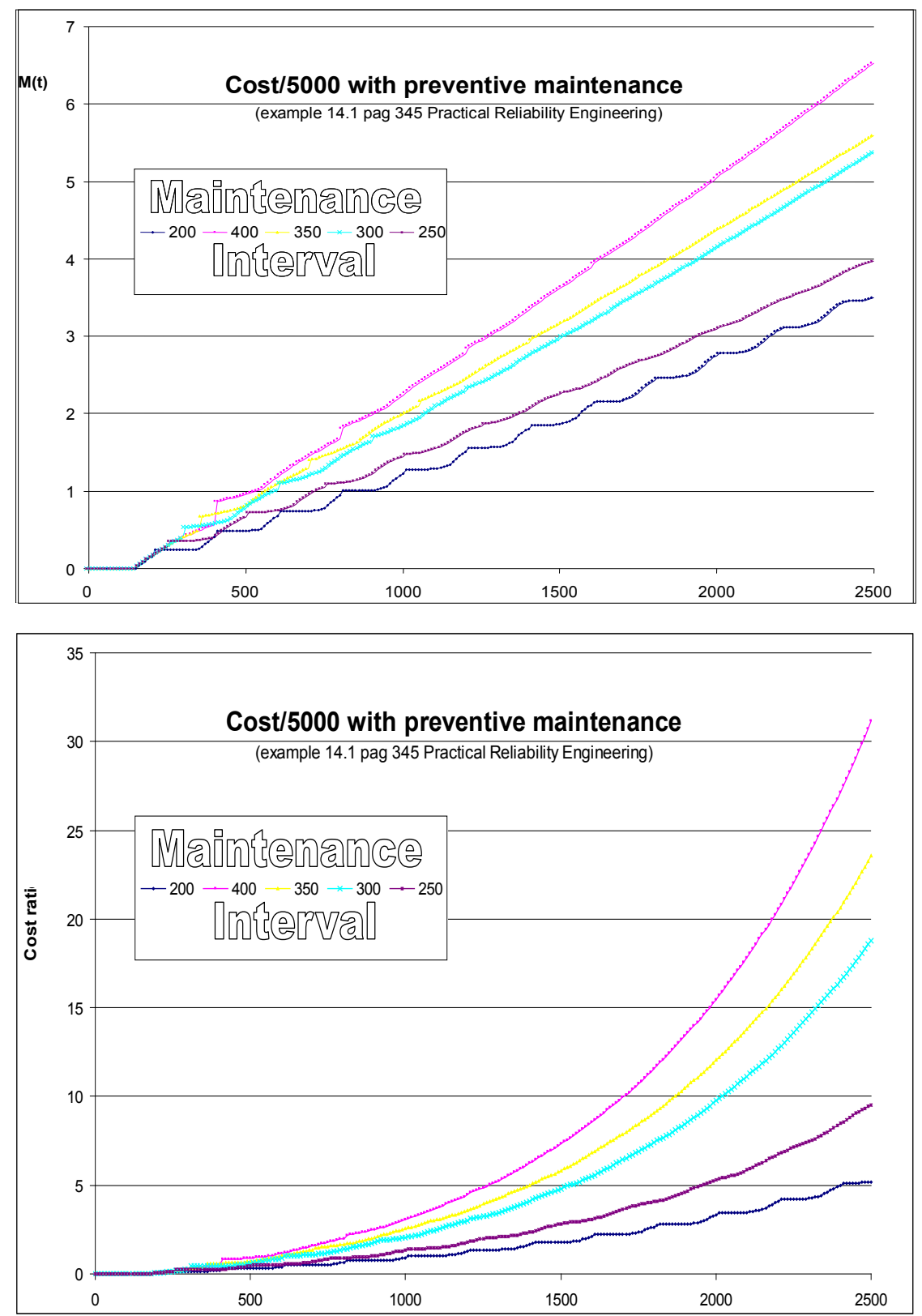

Figure 22. $\mathrm{M}(\mathrm{t})$ and Cost ratio of preventive maintenance

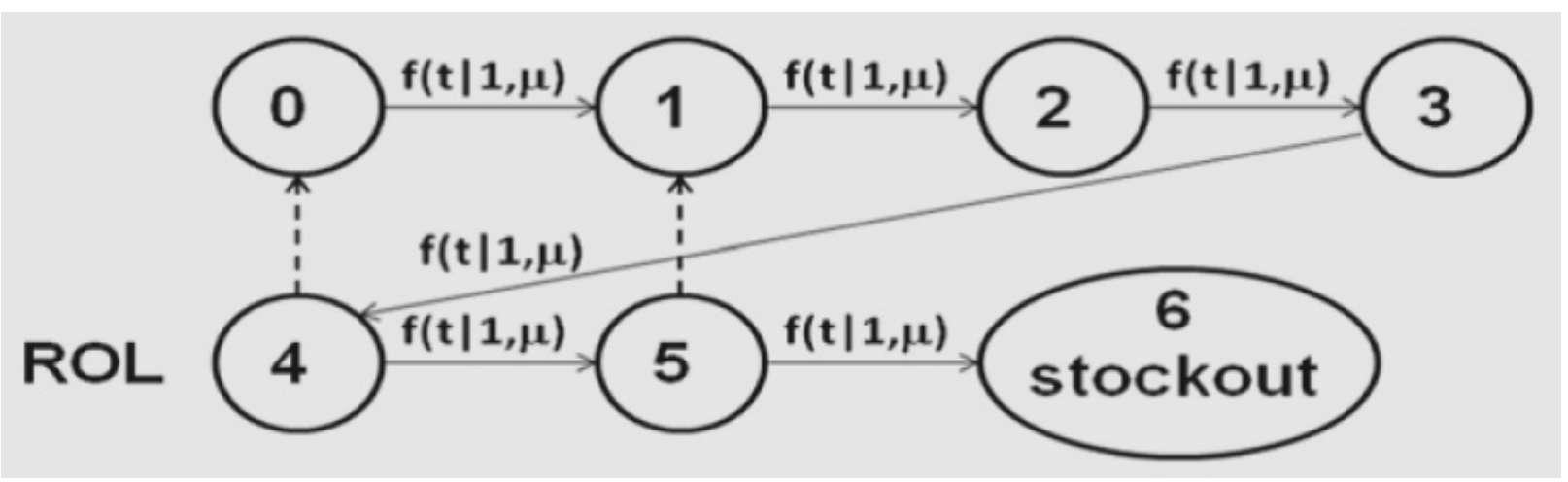

Figure 23. System inventory states (random demand, variable rate); $Q=4, R=2$ 


$$
\begin{aligned}
& R_{0}(t)=1-F(t)+\int_{0}^{t} f(s) R_{1}(t-s) d s \\
& R_{1}(t)=1-F(t)+\int_{0}^{t} f(s) R_{2}(t-s) d s \\
& R_{2}(t)=1-F(t)+\int_{0}^{t} f(s) R_{3}(t-s) d s \\
& R_{3}(t)=1-F(t)+\int_{0}^{t} f(s) R_{4}(t-s) d s \\
& R_{4}(t)=\bar{F}(t) \bar{G}(t)+\int_{0}^{t} f(s) \bar{G}(s) R_{4}(t)+\int_{0}^{t} g(s) \bar{F}(s) R_{0}(t) \\
& R_{5}(t)=\bar{F}(t) \bar{G}(t)
\end{aligned}
$$

This can be written in matrix form as

$$
R(t)=\bar{W}(t)+\int_{0}^{t} B(s) R(t-s) d s
$$

where $b_{i, k}(s) d s$ is the matrix of probability transition from state $\mathrm{i}$ to state $\mathrm{k}$, in the interval $\mathrm{s}^{---} \mathrm{s}+\mathrm{ds}$.

The Service Level $\mathrm{S}(\mathrm{t})$, i.e. no stockout for the interval $0^{---} t$, is $R_{0}(t)$. The probability $P_{i}(t)$ of being in state $i$, at time $t$, is $P_{i}(t)=R_{i}(t)-R_{i+1}(t)$. The integral of $R_{i}(t)$, from 0 to $\infty$, provides the Mean Time To STockOut MTTSTO ${ }_{i}$, when the system begins in state $i$.

The instantaneous Inventory Level I(s), for any time s of the interval $0^{----t}$, and random Lead Time (for replenishment) is $I(s)=\sum_{0}^{5}(R+Q-i) P_{i}(s)$ with cost of Inventory, for the interval 0-----t $I(t)=h \int_{0}^{t} I(s, L) d s$. $I(\infty)=h \int_{0}^{\infty} I(s, L) d s$ is a finite quantity since the probabilities $\mathrm{P}_{\mathrm{i}}(\mathrm{t}) \rightarrow 0$, as $\mathrm{t} \rightarrow \infty$, because it is certain that a stockout happens.

Putting all together we have the relationship that provides the cost $\mathrm{C}(\mathrm{t}, \mathrm{L})$ of the system; the cost rate, usually used in books, is here $\mathrm{C}(\mathrm{t}, \mathrm{L}) / \mathrm{t}$; it is in the following figure 29 . If we

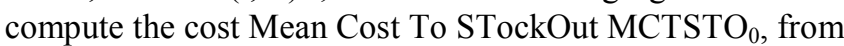
state 0 to the state of stockout, the cost rate is $\mathrm{g}=\mathrm{MCTSTO}_{0} / \mathrm{MTTSTO}_{0}$. [compare this with the findings in RCM...]. This result is valid for any distribution of the time to sell product, from exponential, to Erlang, to ..., ....

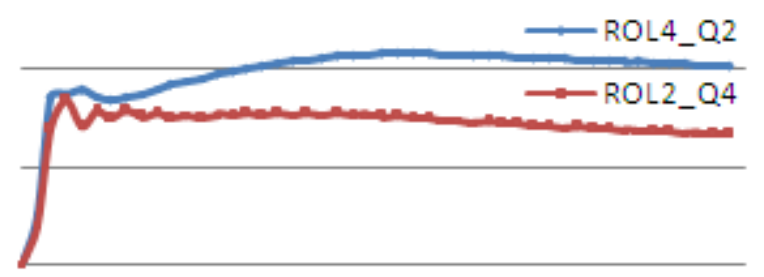

Figure 24. Cost rate of two inventory systems (random demand at variable rate): $\mathrm{Q}=4, \mathrm{R}=2$ vs $\mathrm{Q}=2, \mathrm{R}=4$ [Factory Physics]
As we can see, for the distribution $f(t \mid 1, \mu)$ considered before, the cost rate increases and, after 12 months decreases. The cost rate is lower in the case $Q=4$ and $R=2$, while the service is better for $\mathrm{Q}=2$ and $\mathrm{R}=4$, as we found with the steady state analysis.

Table 10. comparison of cost rate and service level in Inventory Management

\begin{tabular}{|c|c|c|}
\hline $\mathrm{R}=$ & 4 & 2 \\
\hline $\mathrm{Q}=$ & 2 & 4 \\
\hline Cost rate (\$/year) at 6 months & 381.54 & 299.29 \\
\hline Service Level at 6 months & 0.914 & 0.691 \\
\hline
\end{tabular}

If the service level 0.69 , due to the consequences of downtime, is not adequate, we have to choose...

It is therefore very important the interval in which we want optimise.

The best solution for the cost rate is $\mathrm{Q}=4$ and $\mathrm{R}=2$, while for the service level is $\mathrm{Q}=2$ and $\mathrm{R}=4$ (as before).

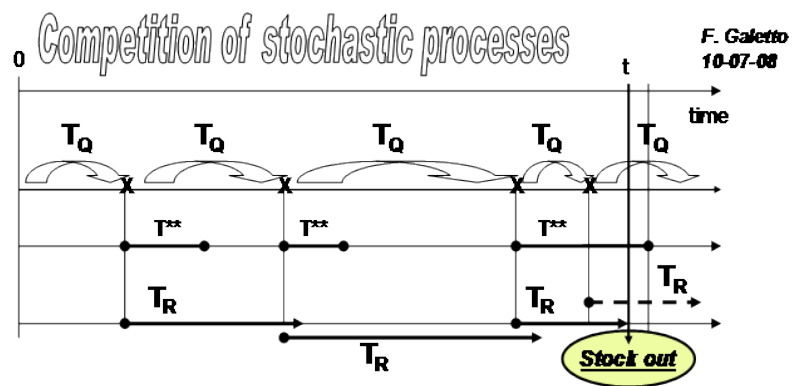

Tr: Lead Tme (r.y.) for transition from Onder to Replenishment with pdf $g(t)$ $T_{\mathbf{Q}}$ : time (r.y.) for selling $Q$ products with pdf f(t)

$T_{R}$ : time (r.v.) for selling $R$ products with pdf fit), for Lead Tme $T^{* *}$ (r.v.)

- $\mathbf{R}_{x}(\mathbf{t})$ : probab-ity of not experiencing Stock out for the interval $0^{-t}$,

if the system entered state $X$, at instant 0

- MTTS : mean time to Stock out, from state $X$

$\square$ probability of not experiencing Stock out are determined by Equations of Reliabitity Integral Theory

Figure 25. the competition of the two stochastic processes, selling and replenishment

This subject has been pointed out many times by $\mathrm{F}$. Galetto, concerning the Preventive Maintenance. We showed it because it has an amazing similarity with inventory management.

We end this paragraph by noting that F. Galetto ideas considered here for the "lost sales" model, or "type 1 Service" model, as can be found in books and papers, being $\lambda$ the rate of demand, always considered as constant !!!!):

- $\mathrm{A} \lambda / \mathrm{Q}$, the "average" order cost per year

- $\quad\left(1-\mathrm{F}_{\mathrm{LT}}(R) \mathrm{p} \lambda / \mathrm{Q}\right.$, the cost of stockout $[\mathrm{p}$ is the cost per stockout, in euro] $\mathrm{F}_{\mathrm{LT}}(\mathrm{R})$ is the CD of demand during the lead time LT, generally ASSUMED as Normally distributed in books and papers

- $\mathrm{h}(\mathrm{R}+\mathrm{Q} / 2-\lambda \mathrm{LT})$, "average" inventory cost per year

are much more suitable for the analysis of practical problems. 
The promise of books and papers, "If stockouts are permitted and lead time is $L T>0$, if demand is random, the formula to be minimised is the average cost rate $=\mathrm{A} \lambda / \mathrm{Q}+\mathrm{h}(\mathrm{R}+\mathrm{Q} / 2-\lambda \mathrm{LT})+\left(1-\mathrm{F}_{\mathrm{LT}}(R)\right) \mathrm{p} \lambda / \mathrm{Q}$ (where $R$ is the trigger quantity for launching the order $Q, p$ is the penalty for stockouts and $F_{L T}(d)$ is the probability distribution of the demand d, during the lead time LT". actually is based on an inconsistent formula, because it is founded on an intuitive [not proved] extension of the formula for $<<$ "constant" demand, no stockouts permitted and lead time zero $>>$.

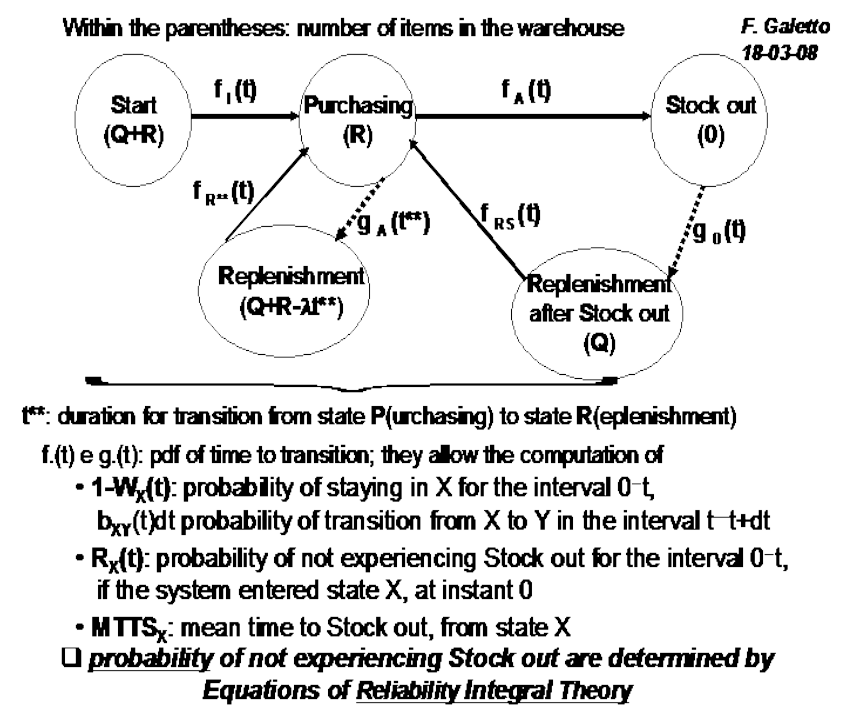

Figure 26. Transitions between the inventory system due to selling and replenishment competition (stochastic processes)

Notice that this formula is the same as that in case of "everything known" and constant!!!!!!!!!!!!. No scientific proof of the formula is ever provided!!! Understanding that the formula is wrong is very easy.

The probability of stockout depends on the competition of two stochastic processes: the demand versus the replenishment. The replenishment density $g(t)$ is not present in any formula you can find in books and papers.

As said before, one can make a Gedanken Experiment, using the Bernoulli's Theorem (equation of motion in hydrodynamics) ...

average cost rate $=\mathrm{A} \lambda / \mathrm{Q}+\mathrm{h}(\mathrm{R}+\mathrm{Q} / 2-\lambda \mathrm{LT})+\left(1-\mathrm{F}_{\mathrm{LT}}(R)\right) \mathrm{p} \lambda / \mathrm{Q}$ is wrong: you can get all that using F. Galetto ideas, as given in his books and papers

Some others cases found in documents Let's consider other examples, with wrong solutions, from various sources.

We saw that Brandimarte and Zotteri, in their book, found $\mathrm{Q}=111, \mathrm{R}=143$ and type 1 Service level=95.73\%, using the following data: $\mathrm{A}=50, \mathrm{~h}=2, \mathrm{p}=500$, Normal Distribution during $\mathrm{LT}=6$ months, with pdf $\mathrm{N}(\mu, \sigma)=\mathrm{N}(100,25)$. They say

We can find the optimal solution $Q^{*}=111$ e $R^{*}=143$ with a type I service level $F(R)=95.73 \%$ by simply iterating the above process.

Using the same data, and the right ideas, F. Galetto finds $\mathrm{Q}=80, \mathrm{R}=92$ and type 1 Service level $=95.73 \%$ : lower costs for the same service level, in the steady state!!!! This result comes from the right formulae.

If we consider a finite horizon, and the density $\mathrm{f}(\mathrm{t} \mid 1, \mu)$, the system faces stockout if $T_{R}<L T$; if, on the contrary, if $T_{R}>$ LT, then the inventory, expired the time LT, raises its quantity by Q, and a new cycle starts. [see Fig. 24]. The findings, for the three methods, are [Lead time assumed constant]:

Table 11. comparison of cost rate in Inventory Management

\begin{tabular}{|c|c|c|c|}
\hline Method & $\begin{array}{c}\text { Formula of the } \\
\text { books }\end{array}$ & $\begin{array}{c}\mathrm{g}=\mathrm{MCTSTO}_{0} / \\
\mathrm{MTTSTO}_{0}\end{array}$ & $\begin{array}{c}\text { using } \\
\mathrm{f}(\mathrm{t} \mid 1, \mu)\end{array}$ \\
\hline $\mathrm{Q}$ and $\mathrm{R}$ & $\begin{array}{c}\mathbf{1 :} \mathrm{Q}=100, \\
\mathrm{R}=141\end{array}$ & $\begin{array}{c}\mathbf{2 :} \mathrm{Q}=100, \\
\mathrm{R}=133\end{array}$ & $\begin{array}{c}\mathbf{3 :} \mathrm{Q}=100, \\
\mathrm{R}=121\end{array}$ \\
\hline $\begin{array}{c}\text { cost }(6 \\
\text { months })\end{array}$ & 603,6 & 567,8 & 492,50 \\
\hline
\end{tabular}

The scientific method provides "Lower costs for the same service level"!! Many other examples could be provided.

How many students, all over the world, are learning wrong methods and will take wrong decisions?

A further example is taken from the Politecnico di Milano course, they say "Buyers Product Company distributes an item known as a Tie Bar, which is U-bolt used on truck equipment. The monthly demand is 11 with standard deviation 3.1.", and they find $\mathrm{Q}=11, \mathrm{R}=19$ and type I Service level $=94.8 \%$, using the following data: $\mathrm{A}=10, \mathrm{~h}=0.022, \mathrm{p}=69$, Normal Distribution during LT=1.5 months, with pdf $\mathrm{N}(\mu$, $\sigma)$. They use a different formula of the one used by Brandimarte and Zotteri, and by Hoop and Spearman but they, as well, do not consider the "pump" and the competition of fig. 25. Using the same data, and the right ideas (as sketched in fig. 24 and 25), F. Galetto finds $\mathrm{Q}=6$, $\mathrm{R}=23$ and type I Service level $=98.2 \%$ : lower costs for better service level!!!! This result comes from the right formulae.

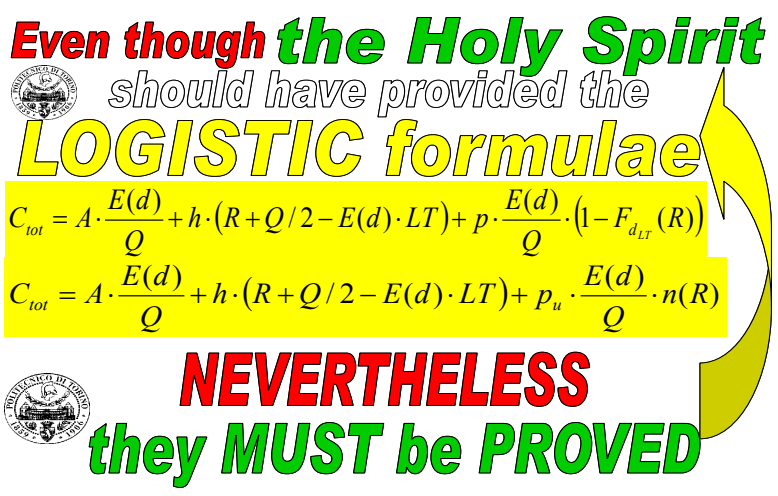

Figure 27. Logistics and Holy Spirit

As a another example, A. Caridi, in his book, provides an example where the weekly demand is Normal distributed with mean 50 and standard deviation 5."; he finds $\mathrm{Q}=294$, $\mathrm{R}=177$ and Service level $=99.9 \%$, using the following data: $\mathrm{A}=30, \mathrm{~h}=1.8, \mathrm{p}=525.4$, Normal Distribution during $\mathrm{LT}=3$ weeks, with pdf $\mathrm{N}(\mu, \sigma)$. He uses a different formula of the one used by Brandimarte and Zotteri, and by Hoop and Spearman but he, as well, does not consider the "pump" of fig. 23. Using the same data and the right ideas (as sketched 
in fig. 25 and 26), F. Galetto finds $\mathrm{Q}=190, \mathrm{R}=133$ and Service level=99.91\%: lower costs for the same service level!!!! This result comes from the right formulae.

As a last example Law and Kelton, in their book Simulation Modelling and Analysis, say "Consider a company which sells a single product and would like to decide how many items to have in inventory for each of the $n$ months. The times between demands are IID exponential random variable with a mean of 0.1 month. The sizes of the demands D are IID random variables (independent of when the demands occur) with probability $P(D=1)=P(D=4)=1 / 6$ $P(D=2)=P(D=3)=1 / 3$. At the beginning of each month, the company reviews the inventory level and decides how many items to order from its supplier." They compare in example 9.2 two inventory policies: $(\mathrm{Q}=40, \mathrm{R}=20)$ and $(\mathrm{Q}=80, \mathrm{R}=20)$ carrying out $10+10$ simulations, and find that the second policy is better. They do not compute the Service level. F. Galetto makes a comparison (only for comparison purpose), using the following data: $A=32, h=1, p=100$, Normal Distribution during LT: the result is opposite, the first one is better; moreover there is a policy $(\mathrm{Q}=5, \mathrm{R}=6)$ much better than both!!! This finding was so peculiar that F. Galetto decided to read thoroughly the book and found in example 9.4 the comparison of 5 alternative policies: $(\mathrm{Q}, \mathrm{R})=(40,20)$, (80, 20), (60,40), (100, 40), (100, 60): Law and Kelton "selected policy 2, $(Q=80, R=20)$ as being the lowest-cost configuration". F. Galetto finds that also doubling all the cost involved, the policy $(\mathrm{Q}=5, \mathrm{R}=6)$ is much better than all the previous policies!!! That sounded still strange and $\mathrm{F}$. Galetto went on: he found this statement in example 12.1 " ... it appears that the smaller values of both $Q$ and $R$ would be preferable, since lower monthly costs are desired": therefore it is rather surprising that, just few pages before, Law and Kelton chose the policy $(\mathrm{Q}=80, \mathrm{R}=20)$ as better than $(\mathrm{Q}=40$, $\mathrm{R}=20$ ). Law and Kelton used simulations, but they, as well, do not consider the "pump". How many students, all over the world, are learning wrong methods and will take wrong decisions?

These examples show very clearly Deming statements:

$\Rightarrow$ "The result is that hundreds of people are learning what is wrong. .... I make this statement on the basis of experience, seeing every day the devastating effects of incompetent teaching and faulty applications." "It is a hazard to copy", "It is necessary to understand the theory of what one wishes to do or to make."

It is important to notice that you do not need the right formula to understand that a wrong formula is wrong. Any formula that does not consider the fig. 25 is wrong! How many professors do not use "pump" in their optimisation? The statement of the Nobel Prize M. Gell-Mann is relevant: "Once that such a misunderstanding has taken place in the publication, it tends to become perpetual, because the various authors simply copy one each other."....>>

\section{Six Sigma}

As said previously the Six Sigma fans claim "6 6 solve every quality problem, allowing big savings for companies that use it"; so in an automotive company [ NOT an Italian one] making disc brakes that needed important improvement appointed a team of five engineers to solve the problem: the problem was "cracks and early wear" of the disks. The basics of $\mathbf{6} \boldsymbol{\Sigma}$ are (wrong...):

"variability is the number one enemy for Quality",

.."A clear commitment to making decisions on the basis of verifiable data, rather than assumptions and guesswork"

"any measurement in the quality field comes from a Normal Distribution", so that

"Using $6 \Sigma$ you get only 3.4 defects per million".

Let $\Phi(\mathrm{x} ; \mu, \sigma)=\mathrm{P}[\mathrm{X} \leq \mathrm{x} ; \mu, \sigma]$ be the Normal distribution of the random variable $X$, LSL e USL the specification limits ( $\mathrm{L}=$ lower, $\mathrm{U}=$ upper) e $\mu \Delta=1.5 \sigma$ allowed deviation of the mean from the real mean $\mu ; P[L S L \leq X \leq U S L ; \mu \pm \mu \Delta, \sigma])=$ $3.4 \mathrm{ppm}$ !

This last statement is true Only and Only IF Normal Distribution is actually applicable AND IF the actual values of $\mu, \mu \Delta, \sigma$ are KNOWN. The Six Sigma fans NEVER say that!!!! They LIE!!! IF we know only the estimates $m, \Delta m$ and $s$ (of $\mu, \mu \Delta, \sigma$ ), as it happens always, the probability $P[L S L \leq X \leq U S L ; m \pm \Delta m, s]$ cannot be computed with the Normal Distribution and is $>>3.4 \mathrm{ppm}[$ it depends on the sample size AND it is $>5000 \mathrm{ppm}$ for sample sizes $>50$ ].

The Six Sigma fans NEVER say that!!!! They deceive you and your intelligence (see section 2!!!)

So we see that, in $6 \sigma$, the Scientific Approach is completely absent from the beginning!!!!

In the $6 \sigma$ applications there is a magic acronym DMAIC used for process improvement: notice process improvement...; it is suggested also by D. C. Montgomery in his very bad book, where he say that (stupidly) "quality is the inverse of variability": so if you produce a product that fails very quickly, you have a very low variability and therefore a very good quality! Compare this with the FAUSTA VIA.

Let's see the data form the very bad book of D. C. Montgomery, an advocate of SIX_SIGMA. It is written there ["example 6-6" (page 291, in the book of Prof. Montgomery)]: "A chemical engineer wants to set up a control chart for monitoring the occurrence of failures of an important valve. She has decided to use the number of hours between failures as the variable to monitor. ... Clearly, time between failures is not normally distributed. [... constructing a time-between-events control chart is essentially equivalent to control charting an exponentially distributed variable.] Figure 6-23 is a control chart for individuals and a moving range control chart for the transformed times between failures [transformed with a Weibull distribution, in order to get Normality!!!!]... If a process change is made that improves the failure rate (such as a different type of maintenance), then we would expect to see the time between failures get longer. ...». This statement proves that Prof. Montgomery (and his lovers, suggesting his book to students) does not realise that, according to his definition, «quality gets 
worse, if a process change is made that improves the failure rate", because "the time between failures get longer». The data are $816,729,4,143,431,8,2837,596,81,227,286,948$, $536,124,603,492,1199,1214,2831,96$ Is it true that HERE "Using $6 \Sigma$ you get only 3.4 defects per million"......... Normal distribution (is not there...) $P[L S L \leq X \leq U S L ; \mu \pm \mu \Delta$, $\sigma])=3.4 \mathrm{ppm} ? ? ? ? ? ? ! ! ! !$

Unfortunately, we do not have space to provide other information on Six Sigma, through real cases.

REMEMBER: I met many of those cited authors: very few knew something about ANOVA, DOE, Reliability, and Preventive Maintenance!!!!

\section{Conclusion}

Any action speaks louder than words: professors teaching wrong ideas do a lot of harm to their students and to the whole Society, although they are all graduated CUM $\angle A U D E$ and with $\mathrm{PhD}(C U M L A U D E)$ and very appreciated by Peer Reviewers who say .....

At the SIX SIGMA lessons attended by Fausto Galetto, one of the lecturer was "very credited": PhD, Visiting Prof. at MIT, author of 9 books, Master Black Belt, ...., director of a Master on $6 \sigma, \ldots$, Winner of the G. Taguchi Award on Robust Engineering, .... Is so good the Director of the Master on $6 \sigma$, met at the SIX SIGMA lessons?' HE knows and teaches wrong ideas. Nevertheless he is ... $\mathrm{PhD}$, Visiting Prof. at MIT, author of 9 books, Master Black Belt, ...., director of a Master on $6 \sigma, \ldots$, Winner of the $G$. Taguchi Award on Robust Engineering, .....

For DFSS Taguchi Methods are called upon... and 3.4 ppm is never mentioned! On the contrary they claim:

Taguchi asserts that robust optimization reduces the failure rate (FR) by a factor of $\left(\frac{1}{2}\right)^{\text {gain/3 }}$, leading to the equation, $\mathrm{FR}_{\text {optimized }}=\mathrm{FR}_{\text {initial }} \times\left(\frac{1}{2}\right)^{\text {gain } / 3}$.

Robust optimization of a good concept is the most effective way to improve reliability. Reliability engineering is more about predicting reliability from data collected from measurements of portions of the product than it is about improving reliability. Condra [9] provides an early application of Taguchi methods as the best way to improve reliability in the context of reliability engineering.

Notice the following figure: they prove that they don't know anything about Reliability, and Preventive Maintenance!!!! NOTICE the Normal distribution.....!!!!!!!!!!!

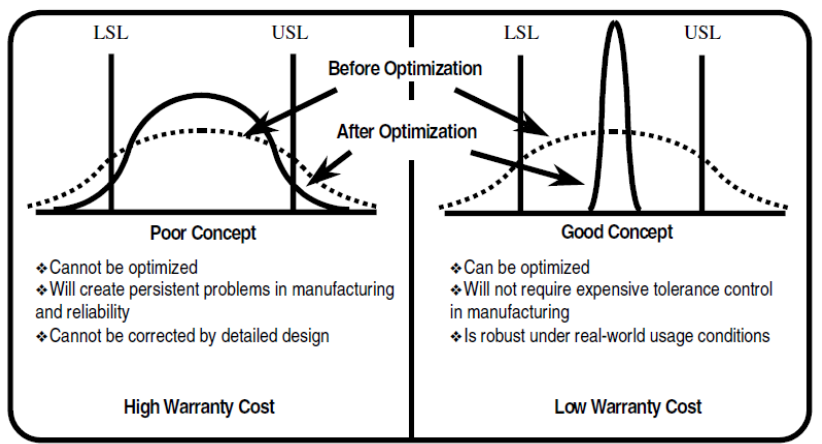

Fausto Galetto challenged G. Taguchi to meet him and prove the truth of his statements, while F.G. had proved his falseness. The meeting was refused.

The QEG "tenors" (lecturers, all graduated CUM LAUDE, and teaching "Quality matters"; they are also in the ResearchGate with high Impact Points!!!) are much credited and have various citations: would that mean that they write good ideas and teach well? The books mentioned above are very credited...: would that mean that their authors write good ideas?

LET'S HOPE that all those incompetent professors will consider their duty to teach scientifically, in order to satisfy the learning need of their students and of the whole society. See Deming, Gell-Mann, Galetto Fausto, ...

None so deaf as he does not want to hear..... Bibliometrics: help or hoax for Quality?

HOAX, if people (professors, managers, consultants, ...) do not use their own brain !!!!!

Here is one excerpt from one of books of a Master Director: I was used to give various cases to my students as problems to be solved at their exams!!!!!(do not mind of the Italian language: the idea of that incompetent Master Director is that you must cancel the data of non-failed items, to be able to estimate the MTTF of the items tested.)

The QEG tenors are on the same line....!!!

YOU student suppose that the test is truncated at $400 \mathrm{~h}$ : estimate the MTTF, WITHOUT neglecting the "suspended items". (the data are time to failure: data $>400$ must be considered as non_failed at 400) [the BMWisti do not know what to do: BMWisti means ....

Misura e analisi dell'affidabilità $\quad 79$

\begin{tabular}{|c|c|c|c|}
\hline $\begin{array}{c}\text { Macchina } \\
\text { di prova }\end{array}$ & $\begin{array}{c}\text { Tempo } \\
\text { al guasto (ore) }\end{array}$ & $\begin{array}{c}\text { Macchina } \\
\text { di prova }\end{array}$ & $\begin{array}{c}\text { Tempo } \\
\text { al guasto (ore) }\end{array}$ \\
\hline 1 & 420 & 12 & 480 \\
\hline 2 & 360 & 13 & 340 \\
\hline 3 & 340 & 14 & 300 \\
\hline 4 & 320 & 15 & 400 \\
\hline 5 & 240 & 16 & 440 \\
\hline 6 & 380 & 17 & 360 \\
\hline 7 & 300 & 18 & 340 \\
\hline 8 & 200 & 19 & 500 \\
\hline 9 & 300 & 20 & 220 \\
\hline 10 & 340 & 21 & 300 \\
\hline 11 & 280 & 22 & 380 \\
\hline
\end{tabular}

Si noti come nelle prove sperimentali si sono considerate solo quelle che siano arrivate al guasto dell'unità. Qualora alcune unità non arrivassero al guasto non è pos- sibile considerare tale dato. Questo genera dei dati che non possono essere considerati ma che comunque generano dei costi di sperimentazione.

Poor students cheated and deceived by the professors met and to be met by them....!

YOU are guilty,

because you do not use your brain!!!!!

"Can you be better than the great professor SSSSSSS?" 


\section{REFERENCES}

[1] Bass, F. M. A new product growth for model consumer durables.Management Science 15 215-227, 1969.

[2] Brandimarte, P., Zotteri, G. Introduction to distribution logistics Wiley, 2007.

[3] Galetto, F. Inventory Management, Spare Parts and Reliability Centred Maintenance for production lines. New Trends in Technology 159-208, 2010.

[4] Jay, A Management and Machiavelli, The Guernsey Press Co., Channel Islands, 1992

[5] Galetto, F. Does "Peer Review" assure Quality of papers and Education?, 9th Conf. on TQM for Higher Education Instutions, Paisley, UK, 2006

[6] Galetto, F. Quality Education versus "Peer Review", IPSI 2006 Montenegro, Montenegro, 2006

[7] Deming, W.E. Out of the crisis, Cambridge Press, 1986

[8] Deming, W.E. The new economics for industry, government, education, Cambridge Press, 1997

[9] Galilei, G.Saggiatore, 1623 (in Italian)
[10] Galilei, G.Dialogo sopra i due massimi sistemi del mondo, Tolemaico e Copernicano (Dialogue on the Two Chief World Systems), 1632 (in Italian)

[11] Berne, E. A che gioco giochiamo (Games people play), Bompiani, Milano, 1987 (in Italian)

[12] Feynmann, R The Character of Physical Law, British Broadcasting Corporation, 1965

[13] Galetto, F.Affidabilità, Teoria e Metodi di Calcolo CLEUP [Padua], 1985 (in Italian)

[14] Galetto, F.Gestione Manageriale dell'Affidabilità, Teoria e Metodi CLUT [Turin], 2004 (in Italian)

[15] Verhulst, P. F. Notice sur la loi que la population poursuit dans son accroissement.Correspondance Mathématique et Physique, 1838, 10, 113-121.

[16] Malthus, T. R. Saggio sul principio di popolazione (1978) a cura di G. Maggioni, Piccola biblioteca Einaudi vol. 312, 1977(in Italian)

[17] Volterra, V. Lecons sur la theorie mathematique de la lutte pour la vie Gauthier-Villars editeurs 1931 